University of Tennessee Health Science Center

UTHSC Digital Commons

\title{
Glioma: A Tale of Corticosteroids, Thiopurines and Proposed Novel AntiGlioma Small Molecules
}

\author{
Amira Ahmed \\ University of Tennessee Health Science Center
}

Follow this and additional works at: https://dc.uthsc.edu/dissertations

Part of the Medicinal and Pharmaceutical Chemistry Commons, and the Pharmaceutics and Drug Design Commons

\section{Recommended Citation}

Ahmed, Amira , "Glioma: A Tale of Corticosteroids, Thiopurines and Proposed Novel AntiGlioma Small Molecules" (2014). Theses and Dissertations (ETD). Paper 8. http://dx.doi.org/10.21007/ etd.cghs.2014.0007.

This Dissertation is brought to you for free and open access by the College of Graduate Health Sciences at UTHSC Digital Commons. It has been accepted for inclusion in Theses and Dissertations (ETD) by an authorized administrator of UTHSC Digital Commons. For more information, please contact jwelch30@uthsc.edu. 


\title{
Glioma: A Tale of Corticosteroids, Thiopurines and Proposed Novel AntiGlioma Small Molecules
}

\begin{abstract}
Glioma is a brain tumor that arises from glial cells or glial progenitor cells, and represents $80 \%$ of malignant brain tumor incidence in the United States. Glioblastoma multiforme (GBM) is the most aggressive primary brain tumor malignancy with fewer than $8 \%$ of patients with GBM surviving for 3 years. Over the past 10 years, despite improvement in diagnosis and therapies for cancer, the survival rate for high-grade glioma patients remains unchanged. The main focus of this dissertation is to investigate two therapeutic agents that are related to increase tumor resistance or increase secondary tumor incidence, which might play a role in poor prognosis and outcome for glioma. There are several studies, which show that corticosteroid treatment increases tumor resistance to chemo- and radiotherapy. Corticosteroids are mainstay drugs for managing peritumoral edema. Therefore, we investigated the effect of corticosteroids in a PDGF driven glioma mouse model. We found that corticosteroid treatment decreases tumor cell proliferation without inducing cell death as shown by a significant decrease in PCNA and Ki67 but not in cleaved caspase-3 staining. Decreased tumor cell growth could compromise the responsiveness of tumor cells to chemotherapy and/or radio-therapy, because cancer therapeutics often target rapidly growing cells. In addition, we found that corticosteroids decrease tumorassociated microglial cells proliferation. Furthermore, in vitro and in vivo studies revealed a shift in microglial cells to less pro-tumor phenotype as evidenced by decreasing MMP9 and IL-1 ra expression. However, we did not find an association between the decrease in tumor proliferation and the shift in microglia phenotype. In addition, the use of thiopurine drugs in leukemia treatment was associated with the development of secondary cancers including glioma. Therefore, we investigated the cytotoxic effects of thiopurine drugs in primary astroglial cultures. Thiopurine drugs are commonly used to treat cancer, autoimmune disorders, and transplantation. However, thiopurines and their metabolites can be inactivated by the thiopurine methyltransferase (TPMT) enzyme. We found that a low TPMT phenotype predicted significantly higher sensitivity to the cytotoxic action of thiopurines than did a high TPMT phenotype. Thiopurines induced significantly more cell death and DNA damage in primary astrocytes and human glioma cells with low TPMT versus high TPMT. It is possible that the DNA damage caused by low TPMT function can ultimately contribute to transformative events over time. Finally, we assessed the anticancer activity of 2 classes of novel small molecules; tetrahydroisoquinoline (THIQ) and chromenes. These molecules were screened in vitro to find the most potent anticancer derivatives that exhibited the least toxicity on normal stromal cells. EDL-360 (5-10 $\mu \mathrm{M})$ and SP-6-27 (IC50 = 7-21 nM) were the most potent analogs of THIQ and chromene in glioma cells, respectively, and low cytotoxic action was observed in normal astrocytes. Furthermore, theses compounds induced a partial regression in glioma tumor engraftment.
\end{abstract}

\section{Document Type}

Dissertation

\section{Degree Name}

Doctor of Philosophy (PhD)

\section{Program}

Pharmaceutical Sciences

\section{Research Advisor}

Duane D. Miller, Ph.D. 


\section{Keywords}

Astrocytes, Brain, Dexamethasone, Glioma, Therapy, Thiopurines

\section{Subject Categories}

Medicinal and Pharmaceutical Chemistry | Medicine and Health Sciences | Pharmaceutics and Drug Design | Pharmacy and Pharmaceutical Sciences

\section{Comments}

One year embargo expired May 2015. 


\title{
Glioma: A Tale of Corticosteroids, Thiopurines and Proposed Novel Anti-Glioma
} Small Molecules

\author{
A Dissertation \\ Presented for \\ The Graduate Studies Council \\ The University of Tennessee \\ Health Science Center
}

\author{
In Partial Fulfillment \\ Of the Requirements for the Degree \\ Doctor of Philosophy \\ From The University of Tennessee
}

By

Amira Ahmed

May 2014 
Portions of Chapter 3 (C) 2011 by PLOS.

Portions of Chapter 4 (C) 2014 by Bentham Science Publishers Ltd and (C) 2012 by Elsevier Ltd.

All other materials $(\mathcal{C} 2014$ by Amira Ahmed.

All rights reserved. 


\section{DEDICATION}

I dedicate this dissertation to my parents; Hosni Ahmed and Samiha Sakran and to my husband; Hossam Abdelsamed. 


\section{ACKNOWLEDGEMENTS}

First I would to thank god and then my parents, especially my father for always believing in me. My father was the source of my inspiration and without his support and ambition I could not have been in the scientific field. Also, without my husband's support, I would not have completed my degree.

Also, I would like to thank Drs. Terreia Jones, Lawrence Pfeffer and Duane Miller for their guidance and mentorship. I wish to thank to my committee members; Drs. Peter McKinnon, Ram Mahato and Zhaohui $\mathrm{Wu}$, for providing a valuable feedback and guidance throughout my Ph.D. training. Also I want to thank Drs. Duane Miller and Shiva Patil research groups for providing the novel anti-glioma small molecules. 


\begin{abstract}
Glioma is a brain tumor that arises from glial cells or glial progenitor cells, and represents $80 \%$ of malignant brain tumor incidence in the United States. Glioblastoma multiforme $(\mathrm{GBM})$ is the most aggressive primary brain tumor malignancy with fewer than $8 \%$ of patients with GBM surviving for 3 years. Over the past 10 years, despite improvement in diagnosis and therapies for cancer, the survival rate for high-grade glioma patients remains unchanged. The main focus of this dissertation is to investigate two therapeutic agents that are related to increase tumor resistance or increase secondary tumor incidence, which might play a role in poor prognosis and outcome for glioma. There are several studies, which show that corticosteroid treatment increases tumor resistance to chemo- and radiotherapy. Corticosteroids are mainstay drugs for managing peritumoral edema. Therefore, we investigated the effect of corticosteroids in a PDGF driven glioma mouse model. We found that corticosteroid treatment decreases tumor cell proliferation without inducing cell death as shown by a significant decrease in PCNA and Ki67 but not in cleaved caspase-3 staining. Decreased tumor cell growth could compromise the responsiveness of tumor cells to chemotherapy and/or radio-therapy, because cancer therapeutics often target rapidly growing cells. In addition, we found that corticosteroids decrease tumor-associated microglial cells proliferation. Furthermore, in vitro and in vivo studies revealed a shift in microglial cells to less pro-tumor phenotype as evidenced by decreasing MMP9 and IL-1ra expression. However, we did not find an association between the decrease in tumor proliferation and the shift in microglia phenotype. In addition, the use of thiopurine drugs in leukemia treatment was associated with the development of secondary cancers including glioma. Therefore, we investigated the cytotoxic effects of thiopurine drugs in primary astroglial cultures. Thiopurine drugs are commonly used to treat cancer, autoimmune disorders, and transplantation. However, thiopurines and their metabolites can be inactivated by the thiopurine methyltransferase (TPMT) enzyme. We found that a low TPMT phenotype predicted significantly higher sensitivity to the cytotoxic action of thiopurines than did a high TPMT phenotype. Thiopurines induced significantly more cell death and DNA damage in primary astrocytes and human glioma cells with low TPMT versus high TPMT. It is possible that the DNA damage caused by low TPMT function can ultimately contribute to transformative events over time. Finally, we assessed the anticancer activity of 2 classes of novel small molecules; tetrahydroisoquinoline (THIQ) and chromenes. These molecules were screened in vitro to find the most potent anticancer derivatives that exhibited the least toxicity on normal stromal cells. EDL-360 (5-10 $\mu \mathrm{M})$ and SP-6-27 $(\mathrm{IC} 50=7-21 \mathrm{nM})$ were the most potent analogs of THIQ and chromene in glioma cells, respectively, and low cytotoxic action was observed in normal astrocytes. Furthermore, theses compounds induced a partial regression in glioma tumor engraftment.
\end{abstract}




\section{TABLE OF CONTENTS}

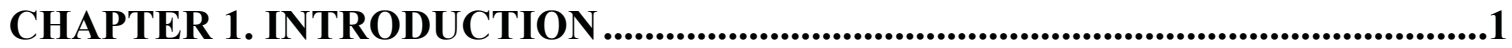

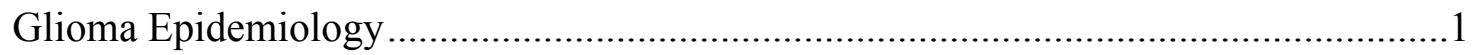

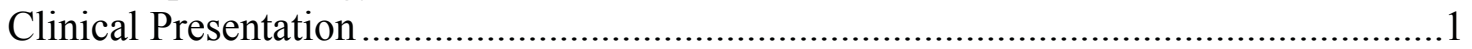

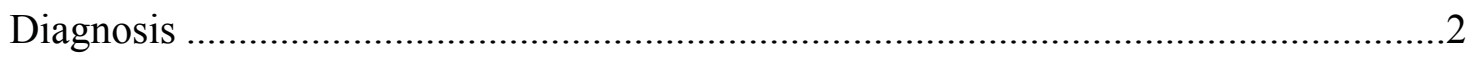

Histopathological and Molecular Classification.........................................................

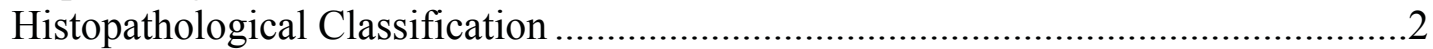

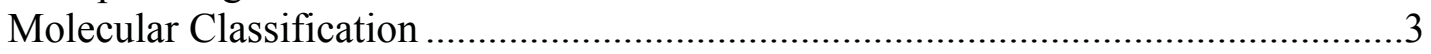

Glioma Treatment

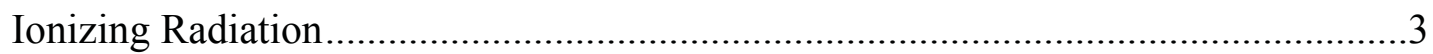

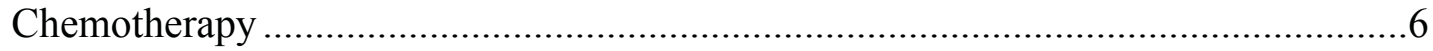

Corticosteroids in Managing Peritumoral Edema........................................................6

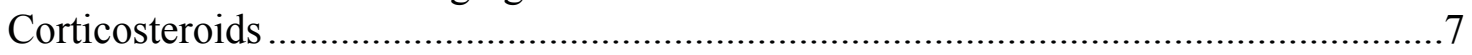

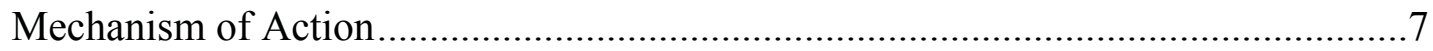

Possible Effects of Corticosteroids on Glioma …………..........................................

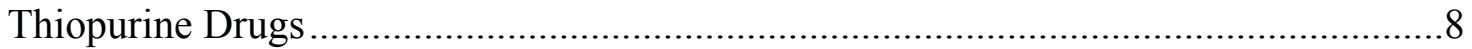

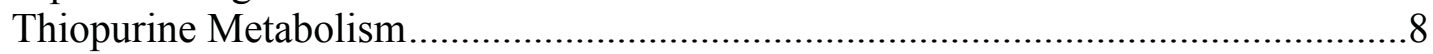

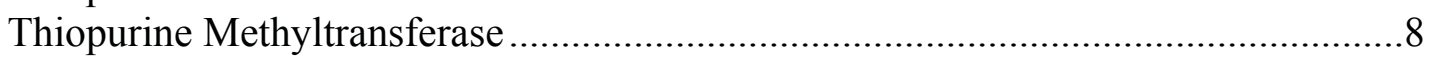

Thiopurines and Incidence of Secondary Brain Tumors ...........................................

\section{CHAPTER 2. THE EFFECTS OF CORTICOSTEROIDS ON GLIOMA AND} MICROGLIAL CELLS .............................................................................................12

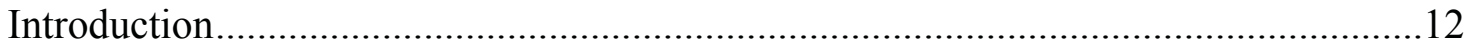

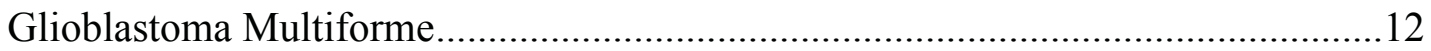

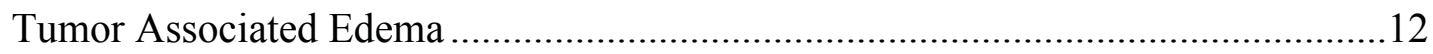

The Effect of Dex on Glioma Cells .....................................................................13

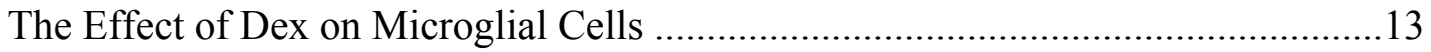

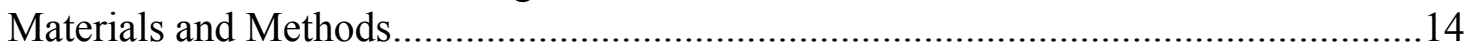

Generation of PDGF Induced Glioma Mouse Model ..............................................14

Primary Glioma Cultures ……………………………...........................................14

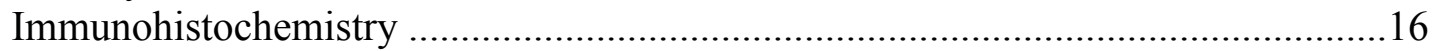

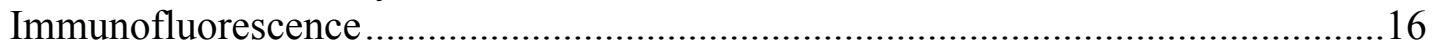

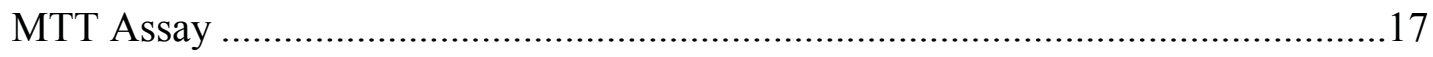

Cell Cycle Analysis.........................................................................................17

Co-Culture Experiments ..............................................................................18

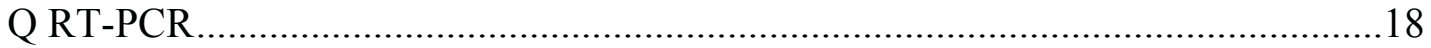

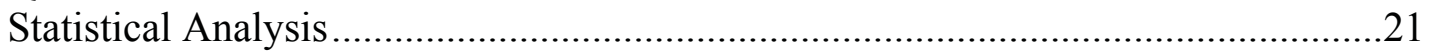

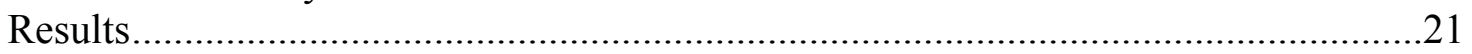

Generation of PDGF Driven Mouse Model of Glioma ..............................................21

Quantification of Cell Proliferation and Cell Death in Dex Treated

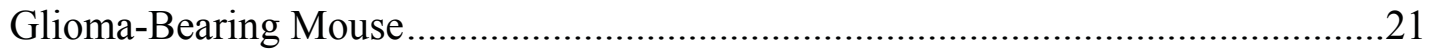

IHC Analysis of Microglial Cell Number and Proliferation Following Dex

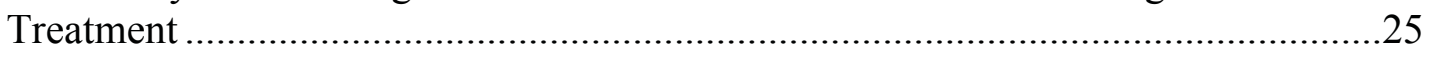


Gene Expression Analysis of Markers Associated with M2- and M1-Like

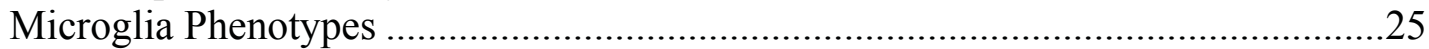

The Effect of Dex on Microglial Cell Polarization....................................................25

In Vitro Proliferation Assessment of Primary Mixed Glioma Cultures.......................30

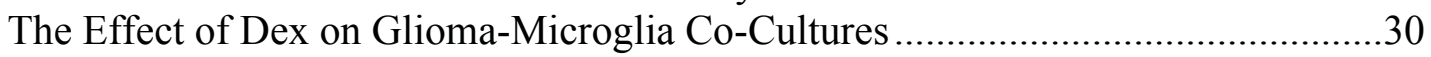

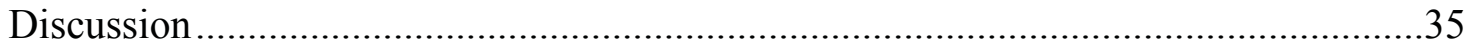

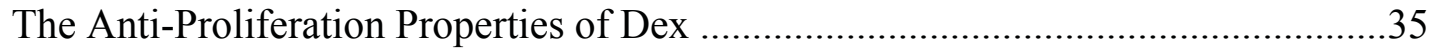

Tumor Associated Microglial Cells Possess M2-Like Phenotype...............................36

Dex Decreases Microglial Cell Polarization to M2-Phenotype ................................. 37

\section{CHAPTER 3. THIOPURINE-INDUCED TOXICITY IN ASTROCYTES IS}

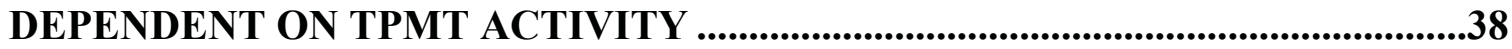

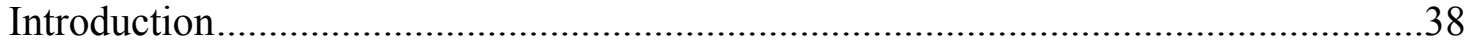

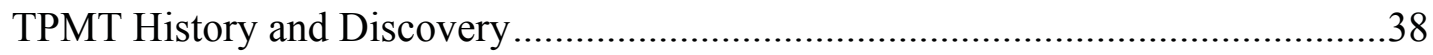

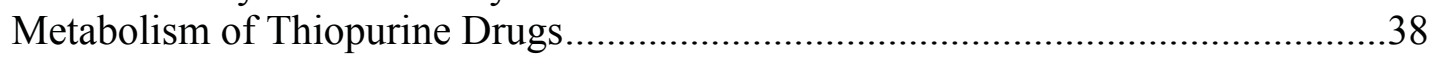

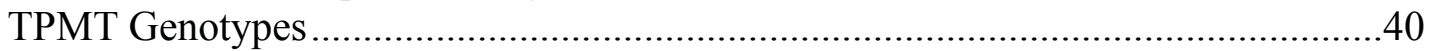

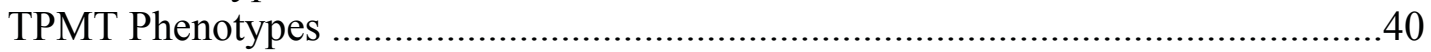

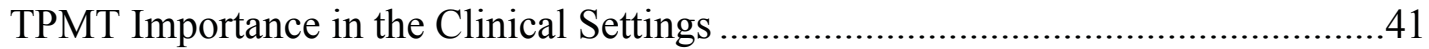

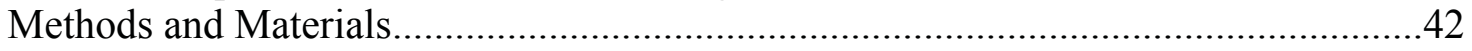

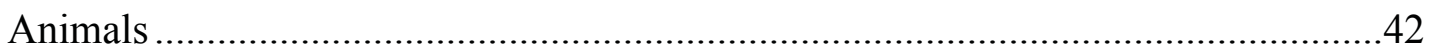

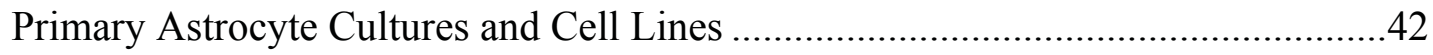

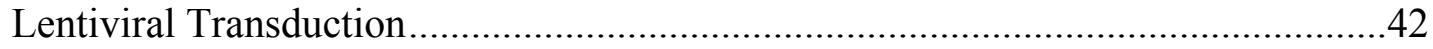

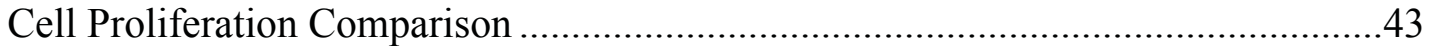

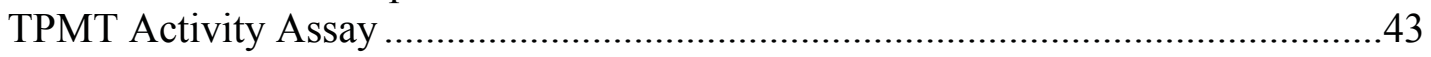

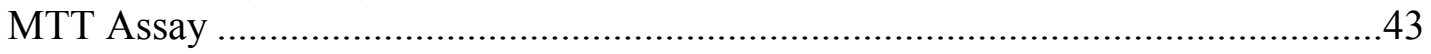

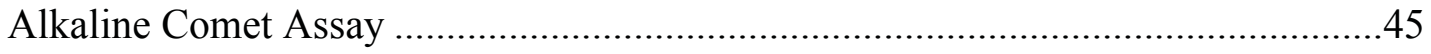

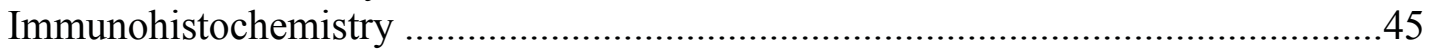

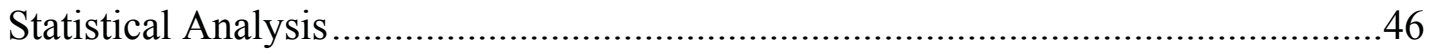

Results.................................................................................................... 46

Characterization of $\mathrm{TPMT}^{+/+}, \mathrm{TPMT}^{+/-}$, and $\mathrm{TPMT}^{-/-}$Primary Mouse Astrocyte

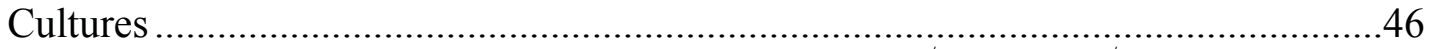

Comparison of TG-Associated Cytotoxicity in $\mathrm{TPMT}^{+/+}, \mathrm{TPMT}^{+/}$, and $\mathrm{TPMT}^{-/}$Primary Astrocyte Cultures ................................................................48

Comparison of the Extent of DNA Damage Between PPMT $^{+/+}, \mathrm{TPMT}^{+/}$, and $\mathrm{TPMT}^{-/}$Primary Astrocytes ........................................................................48

Comparison of TG Phenotypes Between Human Glioma Cell Lines with Different TPMT Protein Activities .................................................................51 Comparison of TG Phenotypes Between A172 ${ }^{\text {mock }}$ and A172 ${ }^{\mathrm{TPMT}}$ Isogenic Cell

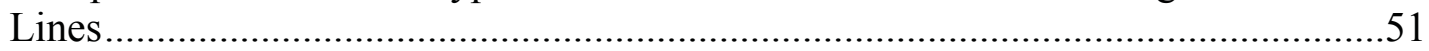

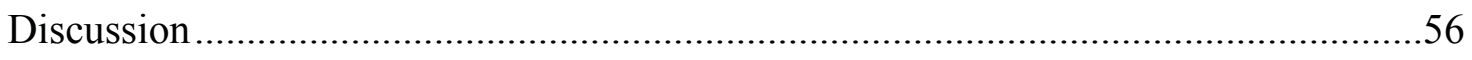

\section{CHAPTER 4. SCREENING OF NOVEL ANTI-GLIOMA SMALL}

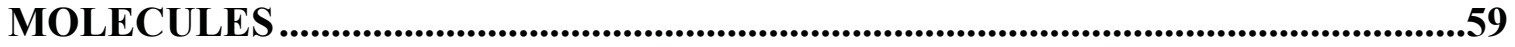

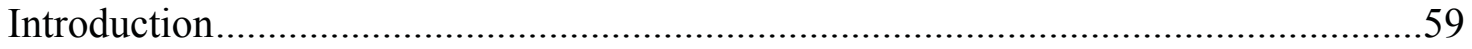

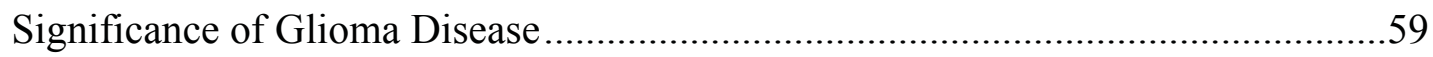

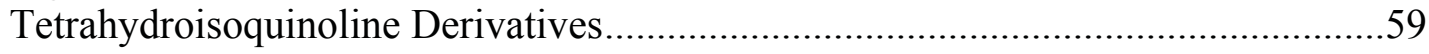




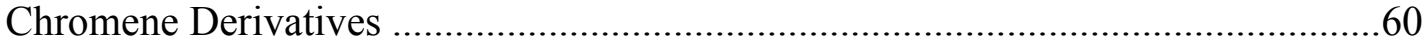

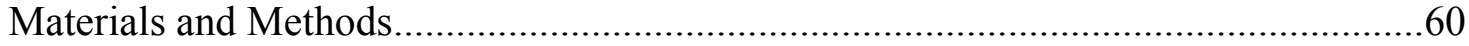

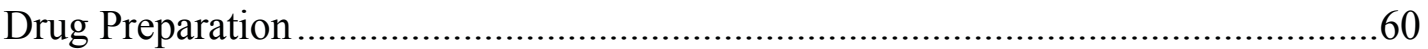

Glioma Cell Lines and Primary Astrocyte Cultures .................................................60

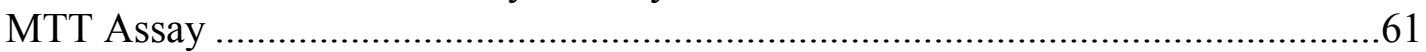

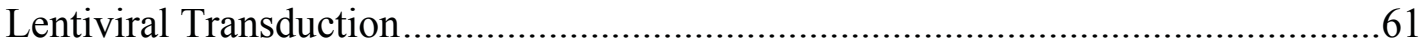

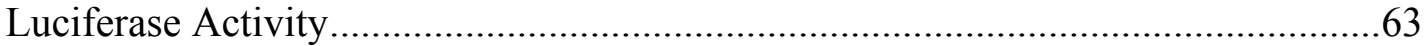

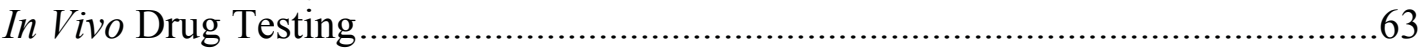

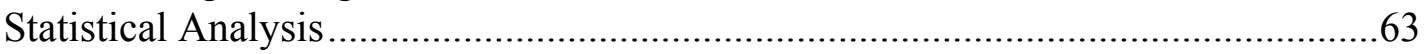

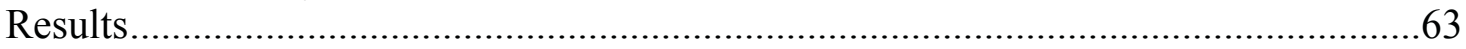

In Vitro Screening of THIQ Analogs......................................................................63

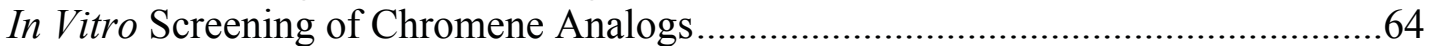

Effect of EDL-360 in Human Glioma Xenograft Mouse Model .................................76

Effect of SP-6-27 in Human Glioma Xenograft Mouse Model ..................................80

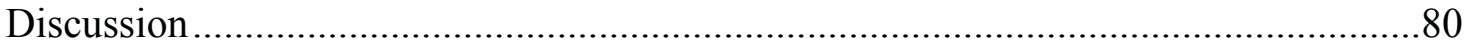

\section{CHAPTER 5. DISCUSSION AND CONCLUDING REMARKS..................................84}

Corticosteroids Might Reduce the Efficacy of Chemotherapy and Radiotherapy.........84

Corticosteroids Decrease Microglial Cell Number and M2-Like Microglial Cells........84

Low TPMT Activity Leads to Increases in TG-Induced Toxicity.................................85

EDL-360 and SP-6-27 Exhibit Anti-Glioma Activity In Vitro and In Vivo ..................87

LIST OF REFERENCES .....................................................................................................89

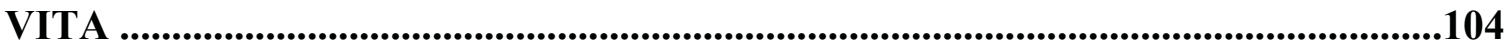




\section{LIST OF TABLES}

Table 1-1. Incidences of secondary neoplasm after thiopurine therapy. .......................10

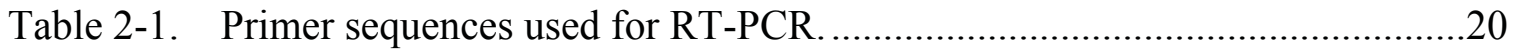




\section{LIST OF FIGURES}

Figure 1-1. Histological and molecular classification of gliomas. .................................4

Figure 1-2. Molecular pathways frequently altered in glioma. ...................................5

Figure 2-1. A cartoon representation of the experimental design. ...............................15

Figure 2-2. A cartoon illustrating tumor-microglia co-cultures. .................................19

Figure 2-3. PDGF-induced mouse model of glioma. .............................................22

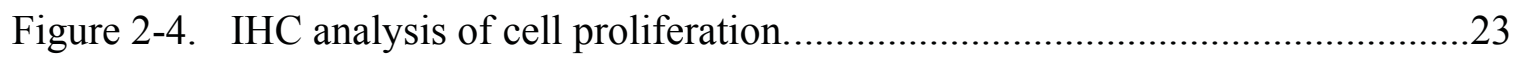

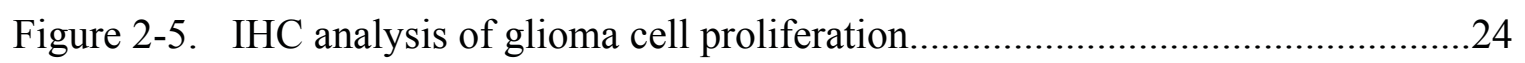

Figure 2-6. IHC analysis of microglial cell number and proliferation following Dex

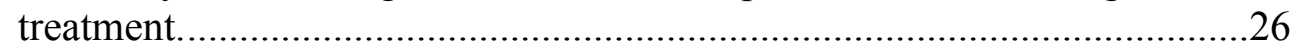

Figure 2-7. Q RT-PCR analysis of the genes associated with M1- and M2-microglial

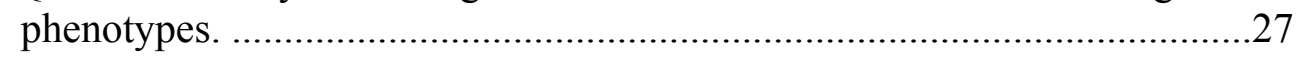

Figure 2-8. Q RT-PCR analysis of genes associated with microglial polarization in control and Dex treated tumor samples...............................................28

Figure 2-9. In vitro cell proliferation assays using primary mixed glioma cultures........31

Figure 2-10. The effect of Dex on glioma-microglia co-cultures..................................32

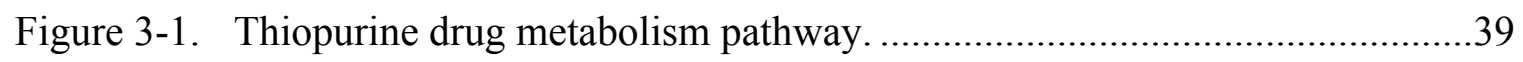

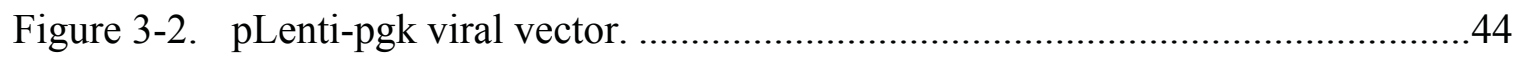

Figure 3-3. Characterization of $\mathrm{TPMT}^{+/+}$, $\mathrm{TPMT}^{+/-}$, and $\mathrm{TPMT}^{-/-}$primary astrocyte

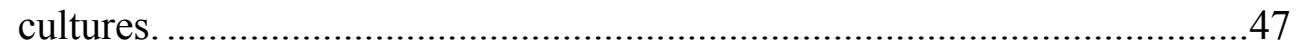

Figure 3-4. Comparison of TG-induced cytotoxicity in primary astrocyte cultures of each TPMT genotype.

Figure 3-5. Comparison of TG-induced genotoxicity in primary astrocyte cultures of each TPMT genotype. ...................................................................

Figure 3-6. Assessment of TPMT-associated phenotypes in human glioma cell lines. ..52

Figure 3-7. TPMT-associated phenotypes in A172 isogenic cells.............................53

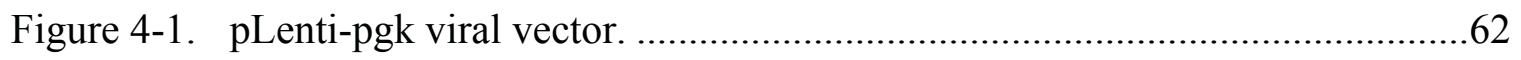


Figure 4-2. The effect of THIQ analogs and TMZ on the cell viability of gliomas

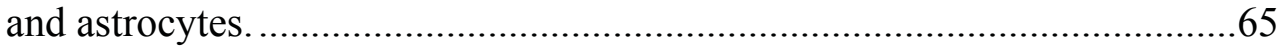

Figure 4-3. The effect of chromene analogs on the cell viability of glioma and

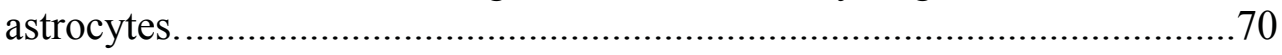

Figure 4-4. Intratumoral treatment with EDL-360 in human glioma xenograft mouse

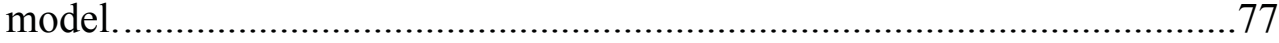

Figure 4-5. Intratumoral treatment with SP-6-27 in human glioma xenograft mouse model. 


\section{LIST OF ABBREVIATIONS}

ALL

ALV

AML

AP-1

AZA

$\mathrm{BBB}$

CBP

CBTRUS

$\mathrm{CM}$

CT

Dex

DSBs

EGFR

FDA

GBM

GFAP

GR

GRE

HDAC2

$\mathrm{H} \& \mathrm{E}$

H-MRSI

HPRT

ICP

IDH1

IL-10

IL-1 ra

IMPDH

LD

MDS

meMP

meTG

meTGN

meTIMP

MGMT

MMR

MP

MRI

MTT

NF1

NSG

PDGFR

PI

PLB
Acute lymphoblastic leukemia

Avian leukosis virus

Actute myloid leukemia

Activating protein 1

Azathiopurine

Blood brain barrier

CREB binding protein

Central brain tumor registry of the United Sates

Conditioned media

Computerized tomography

Dexamethasone

Double strand breaks

Epidermal growth factor receptor

Food and drug administration

Glioblastoma multiforme

Glial fibrillary acid protein

Glucocorticoid receptors

Glucocorticoid responsive elements

Histone deacetylase 2

Hematoxylin and eosin

Proton magnetic resonance spectroscopic imaging

Hypoxanthine phosphoribosyl transferase

Intracranial pressure

Isocitrate dehydrogenase 1

Interleukin-10

Interleukin 1 receptor antagonist

Inositol monophosphate dehydrogenase

Lymphoproliferative disorder

Myelodysplasic syndrome

Methyl-mercaptopurine

Methyl-thioguanine

Methyl-thioguanine nucleotide

Methylthioinosine monophosphate

O6- methylguanine-DNA methyltransferase

Mismatch repair

Mercaptopurine

Magnetic resonance imaging

3-(4,5-dimethylthiazol-2-yl)-2,5-diphenyl tetrazolium bromide

Neurofibromin 1

NOD.Cg-Prkd $c^{\text {scid }} I l 2 r^{t m}{ }^{t m l W j l} / \mathrm{SzJ}$

Platelets derived growth factor receptor

Propidium iodide

Passive lysis buffer 
PNET

PTLD

RCAS

RGD

RLU

SCC

SNPs

SSBs

TCGA

TG

TGMP

TGN

THIQ

TIMP

TMZ

TPMT

TXMP

VDA

WHO
Primitive neuroectodermal tumor

Post-transplantation lymphoproliferative disease

Replication competent ALV splice acceptor

Arginine-glycine-aspartic acid

Relative light units

Squamous cell carcinoma

Single nucleotide polymorphisms

Single strand breaks

The cancer genome atlas

Thioguanine

Thioguanosine monophospahte

Thioguanine nucleotide

Tetrahydroisoquinoline

Thioinosine monophospahte

Temozolomide

Thiopurine methyltransferase

Thioxanthiosine monophosphate

Vascular disrupting agents

World health organization 


\section{CHAPTER 1. INTRODUCTION}

Glioma arises from glial cells or glial progenitor cells, and is the most frequent type of brain tumor [1]. Glial cells, which include astrocytes, oligodendrocytes and ependymal cells, support and maintain normal brain homeostasis. Each of these glial cell types can give rise to a distinctive type of cancer. Glioblastoma multiforme (GBM), however, is the most aggressive primary brain tumor malignancy [1,2]. Despite the fact that GBM is infrequent, it is clinically significant because more than $60 \%$ of the patients die within 1 year from diagnosis [2]. One of the major concerns is glioma resistance to chemotherapy and radiotherapy, which enables tumor cells to repopulate, and thus causing relapse in $50 \%$ of the patients. Recurring tumors are usually more aggressive and resistant to therapy [3]. In the past 10 years, the survival rate remains substantially low for glioma patients. The basic hypothesis to be examined in this thesis is that therapy related resistance and toxicity play a role in the poor prognosis and outcome in glioma. The objectives of this study are:

1- To investigate the effects of corticosteroids (anti-edema) in glioma.

2- To investigate thiopurine (anti-leukemia) induced toxicity in astrocytes with different TPMT phenotypes.

3- To characterize novel small molecules that exhibit anti-glioma activity.

\section{Glioma Epidemiology}

The global incidence of malignant brain tumors is estimated to be approximately 3 in 100,000 per year, with a higher rate in the developed countries compared to the developing countries [4,5]. According to the Central Brain Tumor Registry of the United States (CBTRUS) report, an incidence of 109,695 malignant brain tumors were reported between 2005-2009 [2]. Gliomas represent $29 \%$ of primary brain tumors and $80 \%$ of malignant brain tumors. Three-year relative survival rate of the low-grade diffuse astrocytoma is $55 \%$, while it is less than $8 \%$ of GBM patients. Of note, the mortality rate increases with age. For example, a 5 year relative survival for GBM patients in the fourth decade of their life is approximately $17 \%$, but for the patients in the seventh decade of their life is $1.6 \%$ [2]. In addition, being a white male increases the risk for developing malignant glioma. However, females are at higher risk for non-malignant brain tumors [5].

\section{Clinical Presentation}

At clinical presentation, the most frequent symptoms for newly diagnosed and relapsed glioma patients are headache, behavioral changes, hemiparesis, seizures, nausea and vomiting [6]. Typically, the headache is worse in the morning but gradually alleviates during the day. Nevertheless, brain tumor patients often suffer from changes in personality and cognitive abilities [3]. One of the major concerns is the development of 
neurological symptoms and irreversible neurological damage due to the size of these tumors and their critical location in the brain [3]. In addition to these neurological defects, GBM patients usually suffer from cerebral edema, which is characterized by an increased brain volume due to leakage of the plasma into brain parenchyma (5). Peritumoral edema is considered to be a significant cause of death because it leads to increased intracranial pressure (ICP) [7]. Corticosteroids are often employed to alleviate these neurological symptoms [8], but have been corticosteroid treatment has been associated with various adverse effects that interfere with the quality of life of the patient. The adverse effects include behavioral changes, insomnia, myopathy, hyperglycemia, weight gain, peptic ulcer [9]. Moreover, withdrawal of steroid therapy is also associated with severe side effects including steroid withdrawal syndrome (i.e. headache and lethargy) and adrenal insufficiency due to a lack of the production of endogenous corticosteroids $[9,10]$. The effect of corticosteroids on glioma disease will be discussed in chapter 2.

\section{Diagnosis}

Brain tumor diagnosis starts with non-invasive anatomic imaging techniques such as computerized tomography (CT). X-ray CT scans provide volume imaging by a simultaneous movement of the X-ray source and the film from one position to another. Consequently, tumor location and the associated structure can be identified. The problem with the CT scans, however, is that the images are quite blurry [6,11]. Alternatively, magnetic resonance imaging (MRI) provides a more sensitive detection of small size tumors, location and other abnormal tissue structure. Moreover, it is considered to be safe since it utilizes radio-frequency waves (i.e. non-radioactive). However, it is a more expensive approach than CT scans [12]. Proton Magnetic Resonance Spectroscopic Imaging $\left({ }^{1} \mathrm{H}-\mathrm{MRSI}\right)$ is another advanced imaging tool that provides more accurate information about the location as well as the cellular and metabolic activity of the tumor $[13,14]$. On T2-weighted MRI, low grade astrocytomas appear as hyperintense (bright) area with defined borders [6]. Owing to the infiltrative nature of high grade gliomas, they exhibit irregular contrast enhancement and involve both hemispheres [6]. Although MRI imaging is a valuable diagnostic tool, the final tumor identification and grading is not confirmed until histopathological analysis of a patients' biopsy is performed [3].

\section{Histopathological and Molecular Classification}

\section{Histopathological Classification}

According to the World Health Organization (WHO), gliomas can be histologically classified into several categories based on their morphological resemblance to glial cells. Within each category, the tumors are further graded based on malignancy. The grades are designated by numbers range from "I", for the least malignant tumors, to 
"IV", for most the malignant tumors [15]. For example, astroglial tumors closely resemble astrocytes whereas oligodendroglial tumors resemble oligodendrocytes.

Low-grade piloctic astrocytomas typically arise in the hypothalamic region and usually they are not accompanied with cerebral edema $[6,15]$. However, high-grade astrocytomas, including anaplastic astrocytoma and GBM (Figure 1-1), arise in the cerebral cortex. GBMs are highly heterogeneous and complex in nature, and their incidence peaks in the fifth and the sixth decade of life $[1,6]$. Necrotic regions, hemorrhage, pleomorphic nuclei and vascular proliferation are characteristic of GBM. In addition, GBM highly infiltrates normal tissue forming the secondary structures of Scherer, in which tumor cells surround blood vessels, neurons and subpial zone in the cereberal cortex [16].

\section{Molecular Classification}

Histologically similar tumors can vary significantly on a molecular basis. The Cancer Genome Atlas (TCGA) dataset has been used to classify GBM into 4 molecular subclasses: classical, mesenchymal, proneural, and neural $[17,18]$. The frequently altered oncogenes and tumor suppressor genes in glioma are summarized in Figure 1-2 [19]. The Epidermal growth factor receptor (EGFR) overexpression is frequently altered in the "Classical" subtype of GBM. Moreover, combined deletion of INK4a/ARF and EGFR amplification are observed in $94 \%$ of this subtype. Neurofibromin 1 (NF1) is a tumor suppressor gene that is mutated in $18 \%$ of GBM cases and $53 \%$ of "Mesenchymal" molecular subtype of glioma [18,20]. However, proneural subclass of GBM is characterized by frequent amplification in platelet derived growth factor receptor (PDGFR) (11\%) and point mutations in isocitrate dehydrogenase 1 (IDH1) (30\%). In addition, TP53 and PTEN are altered in 54\% and $16 \%$ of proneural subclass cases [18]. Finally, neural subclass is characterized by neural markers expression; NEFL, GABRA1, SYT1, and SLC12A5. EGFR is also frequently amplified in this subclass but to a lesser extent compared to the classical subtype.

\section{Glioma Treatment}

\section{Ionizing Radiation}

The heterogeneous nature of GBM plays a prominent role in therapeutic resistance [1]. The treatment of brain tumors involves both local and systemic therapeutic approaches. Typically tumor resection is employed to remove the majority of the tumor $[1,3]$. However, complete tumor resection is very challenging due to the diffuse infiltrative nature of these tumors into normal brain parenchyma [21]. As a result, these tumors can repopulate and increase in size significantly after surgery [3]. Cranial irradiation often follows surgical resection. The radiotherapy can alleviate the neurological symptoms and seizures in glioma patients [3]. However, radiotherapy is not 


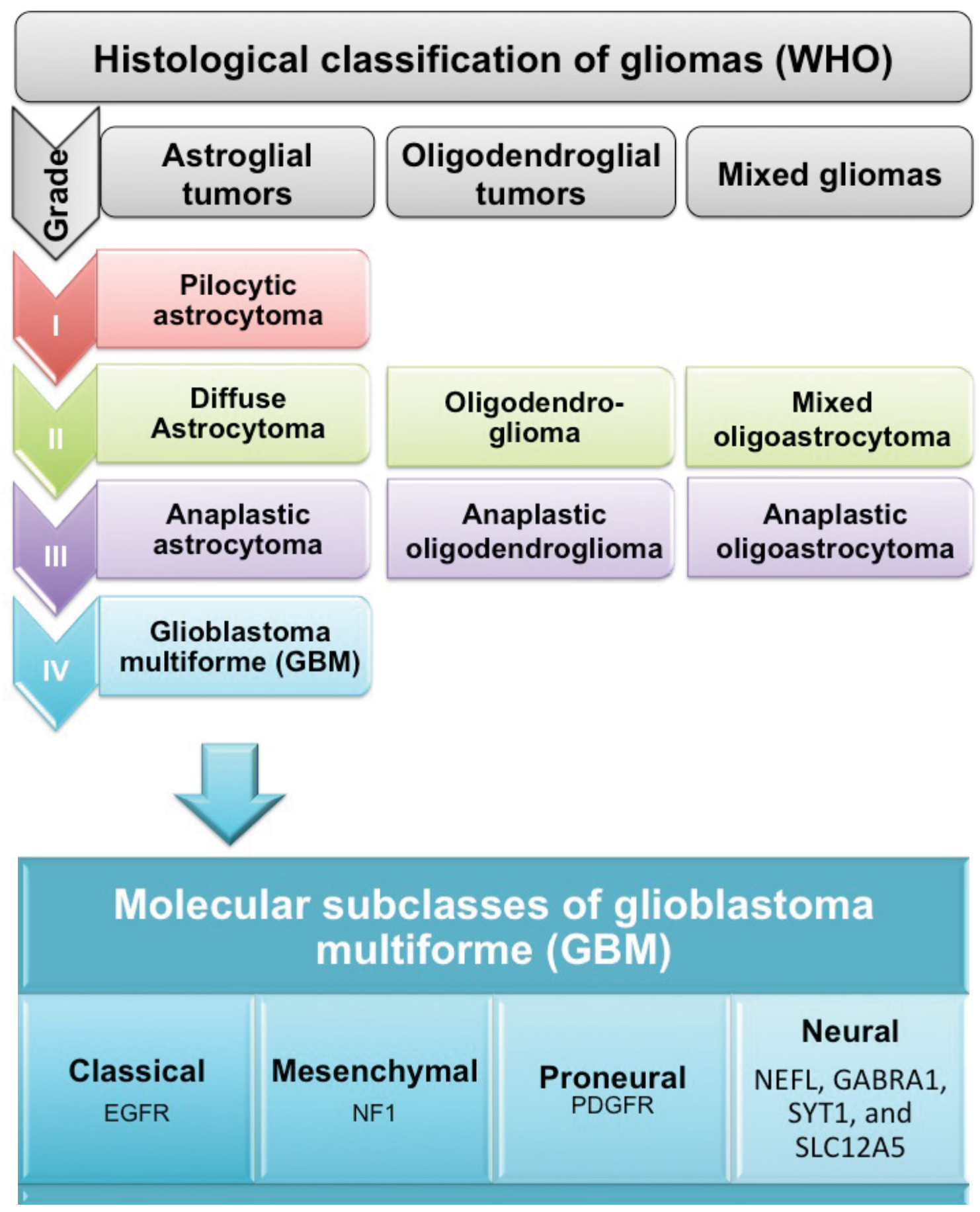

Figure 1-1. Histological and molecular classification of gliomas.

According to the WHO gliomas can be histologically classified into several groups depending on the glial cell of origin. Recent studies further identified 4 molecular subclasses of GBM. 

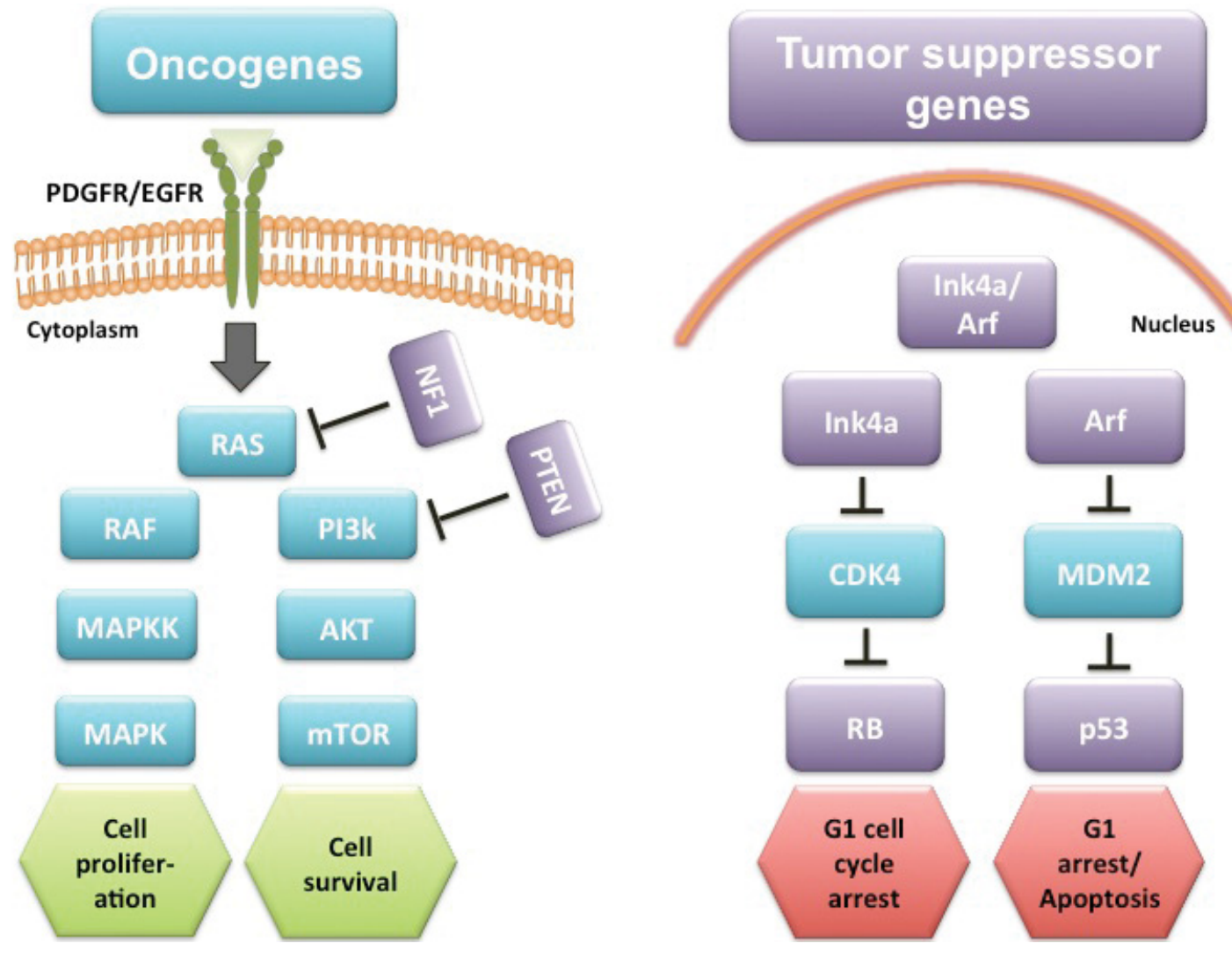

Figure 1-2. Molecular pathways frequently altered in glioma.

Binding of EGF/PDGF growth factors to the EGFR/PDGFR tyrosine kinase receptor leads to the activation of RAS/RAF and RAS/PI3K survival pathways. Tumor suppressor genes NF1 and PTEN inhibit the activity of RAS and PI3K oncogenes, respectively. Ink4a and ARF tumor suppressor genes are frequently mutated in glioma and their loss leads to silencing RB and p53 respectively. Adapted with permission from Patil SA, Hosni-Ahmed A, Jones TS, Patil R, Pfeffer LM, et al. (2013) Novel approaches to glioma drug design and drug screening. Expert Opin Drug Discov 8: 1135-1151. 
recommended for elderly patients ( $>70$ years) because the side effects of irradiation are usually more severe in the elderly. Typically, the radiotherapy regimen is 54-60 Gy given in 30 fractions during 6 weeks of treatment [3]. However, it is not recommended to delay radiotherapy for GBM patients from the time of presentation as it might decrease the overall patient survival rate [22].

\section{Chemotherapy}

Systemic chemotherapy is another treatment option for GBM patients. In 1999, the Food and Drug Administration (FDA) approved Temozolomide (TMZ; Temodar ${ }^{\circledR}$ ) for treating refractory anaplastic astrocytoma, and in $2005 \mathrm{TMZ}$ was approved for GBM treatment. Concurrent treatment of $75 \mathrm{mg}$ of $\mathrm{TMZ} / \mathrm{m}^{2} /$ day and irradiation followed by $\sim 150 \mathrm{mg}$ of $\mathrm{TMZ} / \mathrm{m}^{2} /$ day prolonged the median survival rate to 14.6 months versus 12.1 months for radiotherapy alone [23,24]. This adjuvant TMZ therapy was given 5 days/week for 1 year (28 day cycle) [23]. Of note, the methylation status of O6-methylguanine-DNA methyltransferase (MGMT) promoters is a good predictor of the sensitivity to TMZ therapy. In particular, unmethylated active MGMT enzyme compromises TMZ genotoxicity by removing the alkyl group from the DNA [25]. Therefore, glioma patients with unmethylated MGMT promoter are more resistant to TMZ compared to patients harboring methylated MGMT. Finally, Bevacizumab $\left(\right.$ Avastin $^{\circledR}$ ) is another chemotherapeutic agent that was recently approved for recurrent GBM [24].

\section{Corticosteroids in Managing Peritumoral Edema}

Despite the fact that corticosteroids are not directly employed as anti-neoplastic drugs, they are essential component in managing peritumoral edema. Glucocorticoids are stress hormones that control a wide variety of biological processes such as inflammation, metabolism and behavior [26]. Dexamethasone (Dex), a synthetic corticosteroid, is the most favored corticosteroid drug used by clinicians, whereas the second preferred drug is methylprednisolone. This owes to the long biological half-life of Dex and the minimal mineralocorticoid effect [27]. The dose employed is quite variable depending on patient symptoms. However, the rule of thumb is to use the lowest dose for the shortest period of time to avoid the undesirable side effects of corticosteroids. Additionally, gradual tapering of Dex is critical to avoid corticosteroid withdrawal syndrome. Corticosteroid treatment suppresses the function of the hypothalamic-pituitary-adrenal axis and gradual withdrawal allows the recovery of hypothalamic-pituitary-adrenal axis function [9].

Dex may be used before, during or after radiotherapy, or before or after surgery. At the time of clinical presentation, the starting dose of Dex is $16 \mathrm{mg} /$ day for one week. However, if the patient is unconscious, $40 \mathrm{mg}$ of Dex is given intravenously [8,9]. After 7 days, the dose is adjusted based on patient's symptoms and the extent of tumor resection.

In case the patient's symptoms are improving and the tumor has been surgically removed, the dose is reduced 50\% every 4 days. However, if the symptoms do not improve, the 
dose can be increased up to $100 \mathrm{mg}$ of Dex daily [8]. In addition, radiotherapy treatment can cause a slight increase in brain volume, which might require Dex treatment.

\section{Corticosteroids}

\section{Mechanism of Action}

Corticosteroids achieve their anti-inflammatory effect by acting as a trans-activators or trans-repressors of gene transcription. They are lipophilic substances that can pass through the plasma cell membrane by simple diffusion. Once inside the cell, they bind to glucocorticoid receptors (GR) in the cytoplasm [28]. The hormone-receptor homodimer complex releases the $90 \mathrm{kDa}$ heat-shock protein that allows the complex to translocate into the nucleus [29]. Binding of the complex to the glucocorticoid responsive elements (GRE) on the DNA drives the expression of anti-inflammatory cytokines such as interleukin-10 (IL-10) and interleukin 1 receptor antagonist (IL-1ra) [30]. However, the glucocorticoid-receptor complex can act as a trans-repressor as well by suppressing the expression of pro-inflammatory cytokines. This activity is achieved by interfering with and inhibiting transcription factors, such as NF- $\kappa \mathrm{B}$ and activating protein 1 (AP-1), which drives the expression of pro-inflammatory cytokines [31,32]. Recently, glucocorticoids have also been shown to play a role in chromatin remodeling and hence RNA transcription [33]. GR can bind to and inhibit the function of CREB binding protein (CBP). CBP causes histone acetylation that allows DNA unwinding and hence makes DNA more accessible for transcription machinery. Additionally, GR recruits histone deacetylase-2 (HDAC2), therefore allowing the compaction of DNA and suppression of gene expression $[33,34]$.

\section{Possible Effects of Corticosteroids on Glioma}

Dex is intensively used in the clinic for alleviating peritumoral edema. However, the exact mechanism of how Dex alleviates cerebral edema is unknown. Furthermore, the effect of dexamethasone on tumor cells is controversial. For instance, some studies suggested that it has an anti-proliferative effect in vivo [35]. While other studies have shown that dexamethasone stimulates cell growth at low concentrations and inhibits cell growth at high concentration [36]. This is particularly important because cancer therapeutics often target rapidly growing cells. Therefore, Dex might compromise the responsiveness of tumor cells to DNA damaging agents such as chemo and/or radio-therapies by slowing down tumor cell replication. Indeed, the use of corticosteroid has shown to increase tumor's radioresistance, however, this effect seems to be cell type dependent [37]. Perhaps the upregulation of steroid receptors following ionizing radiation therapy is the mechanism responsible for Dex-induced radioresistance [38]. Such findings highlight the desperate need to understand the specific effect of Dex on tumor cells especially for patients undergoing concomitant chemotherapy or radiotherapy concurrently with corticosteroids. In our studies, we investigated the effect of Dex on 
glioma cell proliferation and death using a genetically engineered mouse model of glioma. However, Dex is not the only drug being associated with severe adverse effect and toxicity. For example the development of secondary tumors in patients has been associated with thiopurine drug treatment.

\section{Thiopurine Drugs}

\section{Thiopurine Metabolism}

Thiopurine drugs (i.e. thioguanine, mercaptopurine, and azathioprine) are commonly used in the treatment of cancer, autoimmune disorders, and transplantation. These agents are pro-drugs that require intracellular conversion to the cytotoxic thioguanine nucleotide (TGN) metabolites by a series of enzymatic reactions to exert their effects [39]. The primary mechanism of thiopurine cytotoxicity is believed to be through the incorporation of TGNs into DNA (DNA-TG) followed by methylation of the DNA-TG bases and subsequent processing by the mismatch repair system [40]. Additionally, inhibition of de novo purine synthesis by methylthioinosine monophosphate (meTIMP) is believed to play some role in how thiopurine drugs work; however thioguanine bypasses the conversion to meTIMP. Alternatively, thiopurines and their metabolites can be inactivated by the thiopurine methyltransferase (TPMT) enzyme.

\section{Thiopurine Methyltransferase}

TPMT is a cytoplasmic enzyme that catalyzes the S-methylation of aromatic and heterocyclic compounds using S-adenosyl-L-methionine as a methyl donor. However, the endogenous substrate for TPMT has not yet been identified [41]. Indeed, TPMT has been studied extensively because of its critical role in the biotransformation of thiopurine agents and because of deactivating polymorphisms that can result in low protein activity $[42,43]$. The genetic polymorphism in TPMT results in a trimodal population frequency distribution in TPMT protein activity. Approximately $90 \%$ of the population have homozygous wild-type TPMT and have high TPMT protein activity; $\sim 10 \%$ are heterozygous with intermediate protein activity; and less than $1 \%$ are homozygous mutant for TPMT and have practically no measurable protein activity. TPMT protein levels are inversely correlated with the cytotoxic TGN metabolites. Hence, low TPMT protein can lead to high levels of intracellular TGNs and life threatening side effects when normal thiopurine doses are administered.

The consequence of low TPMT protein activity is evident in leukemia patient populations who receive thiopurines as part of their antileukemic therapy. When these patients have a low TPMT phenotype and receive normal doses of thiopurines they are at a high risk of life-threatening myelosuppression and secondary cancers [44-46].

Interestingly, one study found that patients who had a low TPMT phenotype and were treated with normal doses of thiopurines concurrently with cranial irradiation as part of 
their antileukemic therapy were at a higher risk of developing brain cancer as compared to patients with normal TPMT $[47,48]$. However, little is known about TPMT function in brain cells. It is plausible that low TPMT function can result in high levels of DNA damage without killing the cells and this might lead to cellular transformation.

\section{Thiopurines and Incidence of Secondary Brain Tumors}

Thiopurine therapy has been associated with cancer development in organ transplantation and autoimmune disorder patients (Table 1-1); however, the importance of TPMT status is unclear. Unfortunately, with the exception of leukemia patients, most patient populations are not evaluated for their TPMT status prior to initiating thiopurine therapy. Since TPMT deficiency is rare across populations, it is difficult to assess the importance of TPMT status to thiopurine therapy-associated cancer risk. Here, we provide a review of the literature of thiopurine drug therapy-related cancers followed by an evaluation of TPMT phenotypes in astroglial cells.

Various types of cancer have been observed in patients who have received long-term thiopurine therapy (Table 1-1). A study conducted by Kandiel et al. found that inflammatory bowel disease patients treated with AZA have a four-fold increased risk of lymphoma [49]. Additionally, there have been several reports suggesting an increased risk of malignancy in renal transplant patients who receive azathioprine, which include cancers of the skin and brain as well as lymphomas and carcinomas [50-52]. The incidence of cutaneous squamous cell carcinoma (SCC) in patients undergoing organ transplantation is 100-fold greater than the normal population [53]. The interaction between UVA radiation and DNA-TGN causes DNA single and double strand breaks possibly due to ROS generation. This in part can explain the high risk of skin cancer in organ transplant patients [54].

Thioguanine and mercaptopurine have been used for childhood acute lymphoblastic leukemia (ALL) treatment for more than 50 years [55,56]. Several studies have reported secondary neoplasms in children treated for ALL including brain cancers, lymphomas, carcinomas and sarcomas [48,57-59]. In a protocol conducted at St. Jude Children's Research Hospital, almost half of the patients having a low TPMT phenotype and who were treated with thiopurines concurrently with cranial irradiation developed brain tumors as a late-complication of their anti-leukemic therapy [47]. This raises the question of whether TPMT can protect important brain cell populations (i.e. astrocytes) from genotoxic events induced by thiopurines. We hypothesize that TPMT deficiency can lead to greater DNA damage and cytotoxicity in astroglial cell populations. 
Table 1-1. Incidences of secondary neoplasm after thiopurine therapy.

\begin{tabular}{|c|c|c|c|c|}
\hline Thiopurines & $\begin{array}{l}\text { Primary } \\
\text { disease }\end{array}$ & $\begin{array}{c}\text { Therapy related } \\
\text { cancer }\end{array}$ & $\begin{array}{c}\text { Number of } \\
\text { patients affected }\end{array}$ & Reference \\
\hline \multirow[t]{8}{*}{ AZA } & $\begin{array}{l}\text { Kidney } \\
\text { transplant }\end{array}$ & $\begin{array}{c}\text { Nodular malignant } \\
\text { melanoma }\end{array}$ & $\begin{array}{l}1 \text { patient in the } \\
\text { case study }\end{array}$ & {$[51]$} \\
\hline & & Brain lymphoma & $\begin{array}{l}25 \text { patients in the } \\
\text { case study }\end{array}$ & {$[52]$} \\
\hline & & Glioblastoma & $\begin{array}{l}3 \text { out of } 5 \text { patients } \\
\text { in the case study }\end{array}$ & {$[60]$} \\
\hline & $\begin{array}{c}\text { Myasthenia } \\
\text { gravis }\end{array}$ & CNS lymphoma & $\begin{array}{l}2 \text { patients in the } \\
\text { case study }\end{array}$ & {$[61]$} \\
\hline & $\begin{array}{l}\text { Inflammatory } \\
\text { bowel disease }\end{array}$ & $\begin{array}{c}\text { Lymphoproliferative } \\
\text { disorder } \Omega\end{array}$ & $\begin{array}{c}3 \text { patients out of } \\
5867\end{array}$ & {$[62]$} \\
\hline & & $\begin{array}{l}\text { Non-Hodgkin's } \\
\text { lymphoma }\end{array}$ & $\begin{array}{l}2 \text { patients out of } \\
212\end{array}$ & [63] \\
\hline & & $\begin{array}{l}\text { Hepatocellular } \\
\text { carcinoma }\end{array}$ & $\begin{array}{l}1 \text { patient in the } \\
\text { case study }\end{array}$ & [64] \\
\hline & & $\begin{array}{l}\text { Hodgkin’s } \\
\text { lymphoma }\end{array}$ & 1 patients out of 1 & {$[65]$} \\
\hline \multirow[t]{10}{*}{ MP } & $\begin{array}{l}\text { Inflammatory } \\
\text { bowel disease }\end{array}$ & $\begin{array}{l}\text { Hodgkin's } \\
\text { lymphoma }\end{array}$ & $\begin{array}{c}1 \text { patient out of } \\
5867\end{array}$ & {$[62]$} \\
\hline & & Lymphoma & 5 patients out of 5 & {$[65]$} \\
\hline & ALL & Brain tumor & $\begin{array}{c}7 \text { out of } 188 \\
\text { patients }^{\dagger}\end{array}$ & [47] \\
\hline & & MDS & $\begin{array}{c}8 \text { out of } 1225 \\
\text { patients }\end{array}$ & {$[66]$} \\
\hline & & PTLD & $\begin{array}{c}1 \text { out of } 1225 \\
\text { patients }\end{array}$ & [66] \\
\hline & & Oral carcinoma & $\begin{array}{c}1 \text { out of } 1225 \\
\text { patients }\end{array}$ & {$[66]$} \\
\hline & & Oral carcinoma & $\begin{array}{c}1 \text { out of } 1225 \\
\text { patients }\end{array}$ & [66] \\
\hline & & Thyroid carcinoma & $\begin{array}{c}1 \text { out of } 1225 \\
\text { patients }\end{array}$ & [66] \\
\hline & & PNET & $\begin{array}{c}1 \text { out of } 1225 \\
\text { patients }\end{array}$ & [66] \\
\hline & & $\begin{array}{l}\text { Intracranial } \\
\text { neoplasms }\end{array}$ & $\begin{array}{l}1 \text { out of } 2 \text { patients } \\
\text { in the case study }\end{array}$ & {$[67]$} \\
\hline
\end{tabular}

Abbreviations:

ALL, acute lymphoblastic leukemia; AML, actute myloid leukemia; AZA, azathiopurine; MP, mercaptopurine; MDS, Myelodysplasic syndrome PNET, primitive neuroectodermal tumor in central nervous system; PTLD, post-transplantation lymphoproliferative disease $\prod$ These three patients received azathiopurine 


\section{Table 1-1. Continued.}

$\Omega$ Lymphoproliferative disorder (LD) includes Anaplastic large cell LD, immunoblastic B-cell lymphoma and B follicular lymphoma

$\dagger$ The incidence of secondary brain tumors in TPMT deficient patients is $42.9 \%$ while in TPMT proficient patients is $8.3 \%$

q Neuroectodermal tumor with astrocytic and neuroblastic nature.

$æ$ This patient received mercaptopurine 


\title{
CHAPTER 2. THE EFFECTS OF CORTICOSTEROIDS ON GLIOMA AND MICROGLIAL CELLS
}

\author{
Introduction
}

\section{Glioblastoma Multiforme}

GBM is one of the most devastating and aggressive types of brain cancer. According to the CBTRUS report, an incidence of 109,695 malignant brain tumors was reported between 2005-2009 [2,68]. Despite the therapeutic advancement, the estimated overall survival is 3 years for approximately only $7 \%$ of the patients [2]. The typical treatment strategy starts with tumor resection to remove the bulk of the tumor. However, complete tumor resection is very challenging due to the diffuse infiltration nature of the tumor into normal brain parenchyma [21]. In addition, tumor recurrence is inevitable for glioma patients, partially because of failure to remove all tumor cells. Surgical resection of the tumor is then followed by aggressive radio-therapy and chemo-therapy (Temozolomide) [69-71].

\section{Tumor Associated Edema}

High-grade gliomas such as GBM are usually associated with cerebral edema, which is characterized by an increase in the brain volume due to leakage of the plasma into brain parenchyma (5). Peritumoral edema is considered to be a significant cause of death because it leads to increased intracranial pressure (ICP) [7]. At clinical presentation, headache, nausea, vomiting and impaired eye movement are the typical symptoms, which substantially impair the quality of life of brain tumor patients $[6,9]$. Since the introduction of Dex, a synthetic glucocorticoid, to medical practice in 1962, there has been outstanding improvement in patients' survival [72]. To date, Dex is the cornerstone for the management of cerebral edema with no superior alternative therapy $[8,9]$. However, Dex has been associated with numerous side effects especially with the long-term use of high doses, including behavioral changes, insomnia, myopathy, hyperglycemia, weight gain and peptic ulcer. Moreover, withdrawal of steroid therapy is also associated with severe side effects including steroid withdrawal syndrome (i.e. headache and lethargy) and adrenal insufficiency due to the lack of production of endogenous corticosteroids $[9,10]$. Therefore, there is an urgent need to discover equal or superior alternatives with fewer side effects. Although how Dex induces its anti-edema effect is largely unknown, it is thought that Dex might achieve its effect by acting on microglial cells [73]. Indeed, microglial secretion of key inflammatory mediators (i.e. matrix metalloproteinases and prostaglandin) that contributes to blood brain barrier (BBB) damage [74,75]. 


\section{The Effect of Dex on Glioma Cells}

Despite the intensive use of Dex for alleviating peritumoral edema, varying effects of Dex on glioma cells in vitro have been found in different cell systems. Grasso et al. demonstrated that $1 \mu \mathrm{M}$ Dex had an anti-proliferative effect on rat glioma cultures [76]. Similarly, ependymoblastoma-bearing mice treated with $40 \mathrm{mg} / \mathrm{kg}$ of Dex had lower tumor size and showed a reduction in thymidine incorporation into DNA [35]. However, the effect of steroids appears to be highly dependent on their concentration. For example, Dex increases the proliferative capacity of human astrocytoma cultures at low concentration, while it is cytotoxic at high concentrations $(>50 \mu \mathrm{M})[36,77]$. For the most part, these studies used non-physiological concentrations of Dex and/or the treatment schedule did not recapitulate the treatment regimen in brain tumor patients. In the current study Dex treatment regimen was carefully chosen to represent its use in the clinical settings. To be able to mimic the in vivo effect and to have reliable meaningful data, our in vitro cell cultures were treated with physiological concentrations of Dex.

\section{The Effect of Dex on Microglial Cells}

To add another layer of complexity there is a dynamic interaction between microglia and tumor cells in the tumor microenvironment. In particular, tumor cells have the capability to silence microglia's immunological responses and shift their polarization to M2-like phenotype [78]. In return M2-like microglia secrete factors that could support tumor proliferation and invasion $[79,80]$. However, the effect of Dex on this symbiotic relationship has not been determined. Since GBM patients have a poor prognosis and very low survival rate, it becomes particularly important to identify the effect of Dex on both glioma cells and stromal cells. Whether Dex influences tumor microglial cell polarization or its consequence on the tumor progression is not precisely identified. It is also unknown whether Dex is interfering with the efficacy of radiotherapy and chemotherapy. Therefore, the aim of our study is to determine the effect of Dex on glioma cell proliferation as well as the effect of Dex on microglial cell number and phenotype.

To our knowledge, this is the first study that utilizes a clinically related glioma mouse model to study the effects of Dex on tumor cells and tumor associated microglial cells. In this model, mouse gliomas recapitulate the histopathological features of human glioma, such as pseudopalisading necrosis, diffuse infiltration and microvascular proliferation [81]. Therefore, the results from this study will be pivotal since it captures several important features of the human disease. The Replication Competent ALV Splice acceptor (RCAS)/ tva system was used to induce PDGF expression in nestin-expressing cells. RCAS is a viral vector derived from the avian leukosis virus (ALV). Because tva receptor is required for viral entry, the mice have been genetically engineered to express the tva receptor in nestin expressing cells (Ntva). After PDGF expression in Ink4a/Arf ${ }^{/-}$ mice, gliomas develop in all the mice within 6 weeks post viral injection [82]. 


\section{Materials and Methods}

\section{Generation of PDGF Induced Glioma Mouse Model}

For theses studies, we employed a PDGF-induced glioma mouse model that was generated in Dr. Eric Holland's laboratory (Fred Hutchinson Cancer Research Center, Seattle), in which the RCAS/tva system to drive the expression of PDGF in nestin expressing cells (Ntva). First, the RCAS/PDGF viral vector (a generous gift from Dr. Holland) was used to infect and overexpress PDGF in chicken fibroblast DF-1 cells (DF-1/PDGF). Mock transfection (DF-1/GFP) was used to monitor infection efficiency with a Nikon Eclipse TE300 fluorescent microscope. Plasmid-FuGENE mixture (1:6) was used to transfect DF-1 cells for $48 \mathrm{hr}$. Subsequently, the cells were passaged and monitored with a fluorescent microscope to determine the transfection efficiency. The cells were maintained in DMEM media (Cellgro, Hemdon, VA) supplemented with 10\% FBS, $2 \mathrm{mM}$ L-glutamine, $100 \mathrm{IU} / \mathrm{ml}$ penicillin, and $100 \mu \mathrm{g} / \mathrm{ml}$ streptomycin. PDGF viral vector producing cells in the log phase (60-80\%) was trypsinized and washed once with PBS. After counting, cells were re-suspended in PBS at 100,000 cells/ $\mu 1$ and placed on ice immediately. Four to six week old Ink4a/ $\mathrm{Arf}^{/-} / \mathrm{Ntva}^{+}$mice (a generous gift from Dr. Holland) were deeply anesthetized with a ketamine/xylene mixture. Using a stereotactic frame, $1 \mu \mathrm{l}$ of cells were intracranially injected into the right frontal cortex. Starting from the 4th week post-injection the mice were weighed daily and monitored for any sign of sickness or neurological symptoms. Mice with symptoms, or those losing weight were placed randomly in the study (Figure 2-1). For in vivo studies, the mice were treated for 3 days with DEX at $10 \mathrm{mg} / \mathrm{kg}$ body weight, which is expected to result in a Dex concentration in brain tissue of $\sim 1 \mu \mathrm{M}[83,84]$. In order to mimic in vivo conditions, the primary cultures were treated with physiologically relevant doses (i.e. $0.1,1$ and $10 \mu \mathrm{M}$ ) of Dex.

\section{Primary Glioma Cultures}

Glioma-bearing mice were sacrificed by transcardiac perfusion using ice-cold PBS. The brain was removed and placed immediately in ice-cold PBS, subsequently the tumor was carefully dissected with surgical tweezers, and single cell suspensions prepared using papain dissociation system. First, the dissected tumor tissue was incubated with papain solution $(0.94 \mathrm{mg} / \mathrm{ml}$ papain, $0.18 \mathrm{mg} / \mathrm{ml}$ EDTA, $0.18 \mathrm{mg} / \mathrm{ml} \mathrm{N}$-Cystein and $0.1 \mathrm{mg} / \mathrm{ml}$ DNase in EBSS; Worthington Biochemical Corp. Lakewood, NJ) for $15 \mathrm{~min}$ at $37^{\circ} \mathrm{C}$. Then, ovomucoid solution $(0.7 \mathrm{mg} / \mathrm{ml}$ ovomucoid, $0.014 \mathrm{mg} / \mathrm{ml} \mathrm{DNase}$ in neuro-basal media) was added to inactivate the enzymatic digestion by papain. After centrifugation at $1200 \mathrm{rpm}$ for $10 \mathrm{~min}$, the cells were washed using neuro-basal media. Subsequently, the cells were re-suspended in DMEM media (Cellgro, Hemdon, VA) supplemented with 10\% FBS, $2 \mathrm{mM}$ L-glutamine, $100 \mathrm{IU} / \mathrm{ml}$ penicillin, and $100 \mu \mathrm{g} / \mathrm{ml}$ streptomycin. 


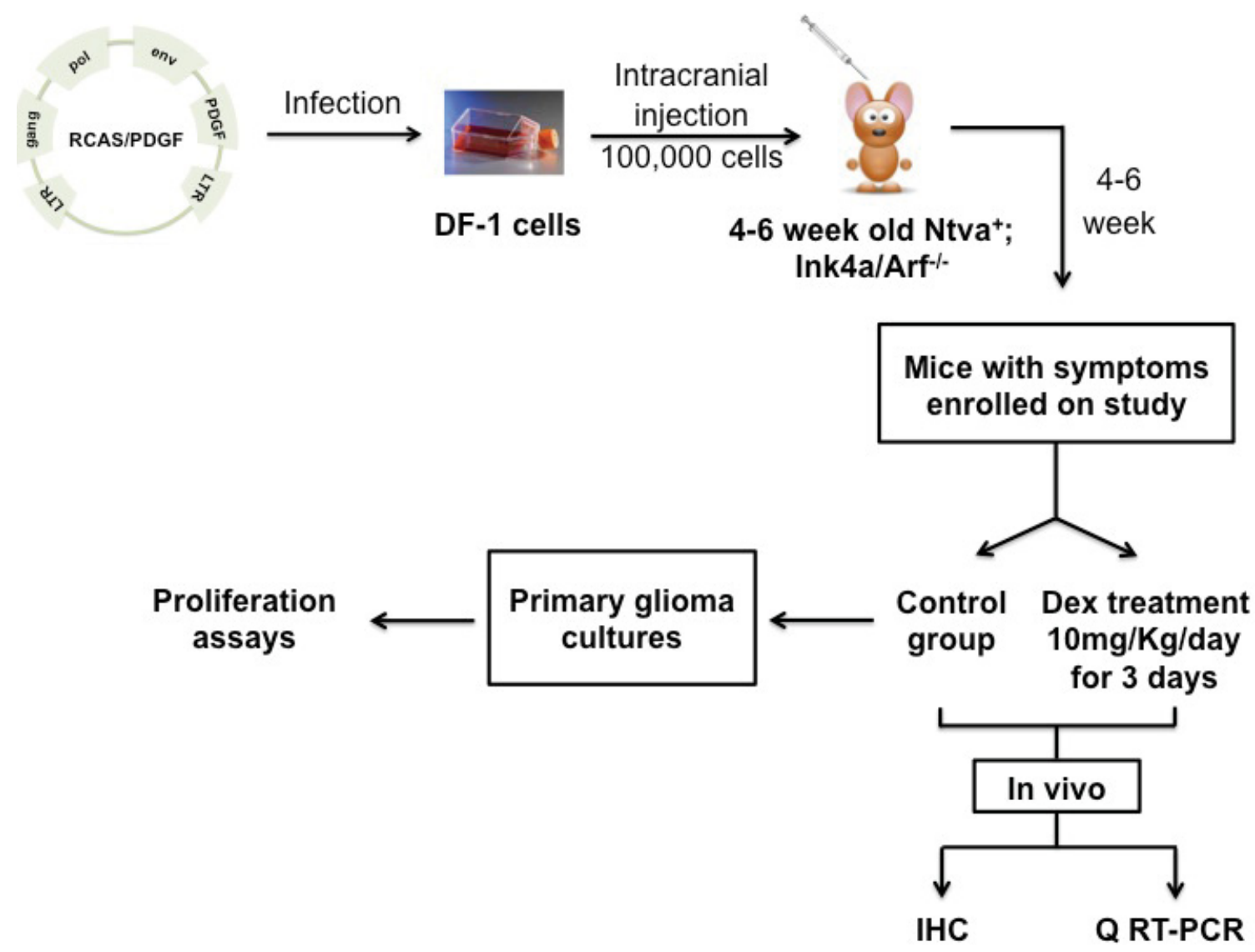

Figure 2-1. A cartoon representation of the experimental design.

RCAS/PDGF vector was used to infect DF-1 chicken fibroblast cells. Virus producing cells were then intracranially injected into $\mathrm{Ntva}^{+}$; Ink4a/Arf ${ }^{--}$. When mice displayed neurological symptoms or had lost weight they were treated with 0 or $10 \mathrm{mg} / \mathrm{kg}$ Dex for 3 days. Tumor samples from control and treated mice were used for IHC and Q RT-PCR experiments. For in vitro testing, primary glioma cultures were generated from control tumor-bearing mice using papain dissociation system and subjected to MTT and cell cycle proliferation assays. 


\section{Immunohistochemistry}

Mice were anesthetized and perfused transcardially with saline followed by $10 \%$ formalin. Brain samples were then post-fixed in neutral buffered $10 \%$ formalin solution (Sigma-Aldrich, St. Louis, MO) for 24 to $72 \mathrm{hr}$. After fixation, tissue samples were blocked with and paraffin embedded for sectioning ( $4 \mu \mathrm{M}$ thick). Following re-hydrating, the slides were microwaved for $10 \mathrm{~min}$ in citrate buffer $(\mathrm{pH}=6.0)$ for antigen retrieval. Then the slides were incubated with 3\% hydrogen peroxide solution for 3 min at room temperature to neutralize endogenous peroxidases. After permeabilization in $0.3 \%$ Triton $\mathrm{X}-100$ in PBS (PBST), the sections were blocked in 2\% BSA, 5\% normal goat serum, $0.1 \%$ Triton $\mathrm{X}-100$ in PBS for $1 \mathrm{hr}$ at room temperature. Primary antibodies were incubated overnight at $4^{\circ} \mathrm{C}$ at a dilution of 1:500 (Iba-1; Wako Chemicals Inc. Richmond, VA), 1:300 (Cleaved caspase-3; Cell Signaling Technology, Beverly, MA), 1:200 (Ki67; Vector Laboratories, Burlingame, CA) and 1:2000 (PCNA; Dako, Carpinteria, CA). After rinsing the slides 3 times in $0.1 \%$ PBST, the slides were incubated with biotinylated goat anti-rabbit secondary antibody (Vector laboratories, Burlingame, CA) for Iba-1, cleaved caspase-3, and Ki67. MOM kit (Vector Laboratories, Burlingame, CA) was used for PCNA staining. The slides were then incubated with $\mathrm{ABC}$ solution (Vectastain ABC Elite kit; Vector Laboratories, Burlingame, CA) for 30 min, washed 3 times in water and then incubated with DAB solution (Vector Laboratories, Burlingame, CA) until the brown color developed. After counterstaining the nucleus with hematoxlin

(Richard-Allan Scientific, Kalamazoo, MI), the slides were dehydrated and mounted with permount mounting media (Fisher Scientific Co., Fair Lawn, NJ). Finally, the slides were scanned with Aperio Spectrum system and analyzed using ImageScope v11.1.2.760

(Aperio, Vista, CA). The whole tumor regions were selected based on H\&E staining and positive pixels and negative pixels (unstained nuclei) were counted. Percent positivity was calculated based on Equation 2-1;

$$
\% \text { Positivity }=(\text { positive pixels } * 100) / \text { total pixels }
$$

To quantify of areas of necrosis, paraffin embedded tumor sections were stained with hematoxylin and eosin (H\&E). Subsequently, the slides were scanned using Spectrum system from Aperio and analyzed using ImageScop v11.1.2.760 (Aperio Vista, $\mathrm{CA})$. The tumor region and regions of necrosis were selected and the necrotic index was calculated according to Equation 2-2 [85];

Necrotic index $=\left(\right.$ Areas of necrosis $\left.\left(\mu \mathrm{m}^{2}\right)^{*} 100\right) /$ Total area of tumor section $\left(\mu \mathrm{m}^{2}\right)$

(Eq. 2-2)

\section{Immunofluorescence}

Immunofluorescence double staining was performed on re-hydrated brain sections. The slides microwaved for $10 \mathrm{~min}$ in citrate buffer $\mathrm{pH} 6.0$ for antigen retrieval. After permeabilization in $0.3 \%$ triton X-100 in PBS (PBST), the sections were blocked in $2 \%$ BSA, 5\% normal donkey serum, $0.1 \%$ triton $\mathrm{X}-100$ in PBS for $1 \mathrm{hr}$ at room 
temperature. Anti-Iba-1 (1:250; Wako Chemicals Inc. Richmond, VA) or anti-olig2 (1:100; Millipore Co., Bedford, MA) primary antibodies were incubated overnight at 4 ${ }^{\circ} \mathrm{C}$. After rinsing the slides 3 times in $0.1 \%$ PBST, the slides were incubated with Alexa Fluor 555 conjugated goat anti-rabbit secondary antibody (1:500; Molecular Probes Life Technologies Inc., Grand Island, NY). The slides were then washed 3 times in $0.1 \%$ PBST and incubated with the anti-PCNA primary antibody (1:100; Dako, Carpinteria, CA) for $1 \mathrm{hr}$. Subsequently, the sections were incubated for $1 \mathrm{~h}$ at room temperature with Alexa Fluor 448 conjugated goat anti-mouse secondary antibody (1:500; Molecular Probes Life Technologies Inc., Grand Island, NY). Finally, vectashield mounting media (Vector Laboratories, Burlingame, CA) containing DAPI was used to stain nuclei. Slides were then visualized on a LSM Zeiss 700 microscope, 20X objective, $2 \mathrm{X}$ zoom. At least 200 cells were analyzed per slide for Olig2 ${ }^{+}$staining and 50 cells for $\mathrm{Iba}_{-1}{ }^{+}$staining.

The percentage of Iba-1 positive cells were quantified in primary glioma cultures by immunocytochemical analysis. Cells were fixed with 4\% PFA/PBS for $10 \mathrm{~min}$, and permeabilized with $0.5 \%$ Triton X-100/PBS for 5 min. Cells were then washed with PBS and blocked with $3 \%$ BSA/PBS for $1 \mathrm{~h}$. Cells were immunostained with anti-Iba-1 (1:250; Wako Chemicals Inc. Richmond, VA) for $1 \mathrm{~h}$. Subsequently, cells were washed with PBS and incubated for $1 \mathrm{~h}$ with in Alexa Fluor 555 conjugated goat anti-rabbit secondary antibody (1:500; Molecular Probes Life Technologies Inc., Grand Island, NY). Finally, vectashield mounting media (Vector Laboratories, Burlingame, CA) containing DAPI was used to stain nuclei. Slides were visualized using Nikon Eclipse TE300 fluorescent microscope. A minimum of 100 cells were analyzed per culture for Iba-1 staining using Adobe Photoshop CS4.

\section{MTT Assay}

Cell viability was measured using 3-(4,5-dimethylthiazol-2-yl)-2,5-diphenyl tetrazolium bromide (Sigma-Aldrich, St. Louis, MO). Primary glioma cultures were seeded at a density of 1000 cells/ well in 96-well plates. A $50 \mu 1$ stock solution of Dex (Sigma-Aldrich, St. Louis, MO) was prepared by dissolving Dex in DMSO. Cells were allowed to attach overnight and Dex or vehicle control was added to the appropriate wells. After 3 days of drug exposure, MTT was added to wells and allowed to incubate for $3 \mathrm{hr}$. Afterwards, the media was carefully aspirated and the purple formazan crystals were dissolved in $100 \mu 1$ DMSO (Fisher Scientific Co., Fair Lawn, NJ). Absorbance was measured at wavelength of 570/690 nm using a FLx800 fluorescence microplate reader (BioTek Instruments, Inc., Burlington, VT).

\section{Cell Cycle Analysis}

The effect of Dex on cell cycle phase distribution was measured by flow cytometry. Cells seeded on $10 \mathrm{~cm}$ plates at $4000 / \mathrm{ml}$ were allowed to attach to the plates overnight and then treated with $0.1,1$ and $10 \mu \mathrm{l}$ Dex for 3 days. After cell trypsinization and washing in ice cold PBS, the cells were fixed in ice cold $70 \%$ ethanol for at least 1 
hr. Subsequently, the cells washed with PBS and stained with $0.05 \mathrm{mg} / \mathrm{ml}$ propidium iodide (PI) in presence of $0.1 \mathrm{mg} / \mathrm{ml}$ RNAses and then analyzed using BD Accuri C6 flow cytometer (BD Bioscience, San Jose, CA). A quantification of different phases of cell cycle (G0/G1, S, G2/M) was carried out using Modfit LT V2.0 (Verity Software House, Tosham, ME).

\section{Co-Culture Experiments}

To determine the role of microglia in tumor cell proliferation, co-cultures of microglia-tumor cells were generated. First, primary glioma cells were generated form control tumor-bearing mice using papain dissociation system. Since, microglial cells attach very well to the plate and are hard to trypsinize, we used this property to deplete them by mild trypsinization and serial subculturing technique. Furthermore, microglial cell content was determined using ICC. Glioma co-cultures containing $0 \%, 5 \%$ or $10 \%$ BV2 microglial cells were generated (Figure 2-2). Pure glioma cultures and microglia cultures were used as controls. For MTT cell viability assay, 250 cells/well in $96-w e l l$ plates were seeded and treated with $0,0.001,0.01,0.1,1,10,100 \mu \mathrm{M}$ Dex for 3 days. Additionally cell cycle assay was employed on single and co-cultures after 3 days of Dex treatment with either 0 or $10 \mu \mathrm{M}$. Cell proliferation assays were performed as mentioned above.

\section{Q RT-PCR}

Glioma conditioned media (CM) was obtained by growing 1 X10 $0^{5}$ glioma cells $/ \mathrm{ml}$ of a complete media without antibiotic for $48 \mathrm{hs}$. The collected CM was filtered $(0.2 \mu \mathrm{m}$ filter) to remove cellular materials and stored at $-20^{\circ} \mathrm{C}$. BV2 microglial cells were seeded at $4 \times 10^{4}$ cells $/ \mathrm{ml}$ in the $\mathrm{CM}$ and incubated overnight to allow cell attachment. The cultures were treated with 0 or $10 \mu \mathrm{M}$ Dex for 3 days. Total RNA was extracted from BV2 cell cultures and mouse tumor samples using Trizol reagent (Invitrogen, Carlsbad, CA). The yield and purity of the RNA was confirmed using Nanodrop; ND-1000 V3, spectrophotometer.

First, cDNA was synthetized using transcriptor first strand cDNA synthesis kit (Roche Applied Sciences, Indianapols, IN) using $1 \mu \mathrm{g}$ of RNA. Primer panels were designed using Roche Universal Probe Library to detect M1- (iNOS, IFN $\gamma$, IL-1 $\beta$, TNF $\alpha$, CD86) and M2- (Arg1, TGF- $\beta$, CD204, MMP9, IL-1ra) markers of microglia (Table 2-1). The housekeeping gene, TBP, was used to normalize genes. Expression Q RT-PCR was performed using LC480 light cycler under the following conditions; 50 cycles of amplification, activation step for $5 \mathrm{~min}$ at $95^{\circ} \mathrm{C}$, annealing step for $30 \mathrm{sec} 60^{\circ} \mathrm{C}$ and finally extension step $72^{\circ} \mathrm{C}$ for $10 \mathrm{sec}$. 


\section{Primary glioma culture}

\section{Sub-culturing}

Tumor

cells
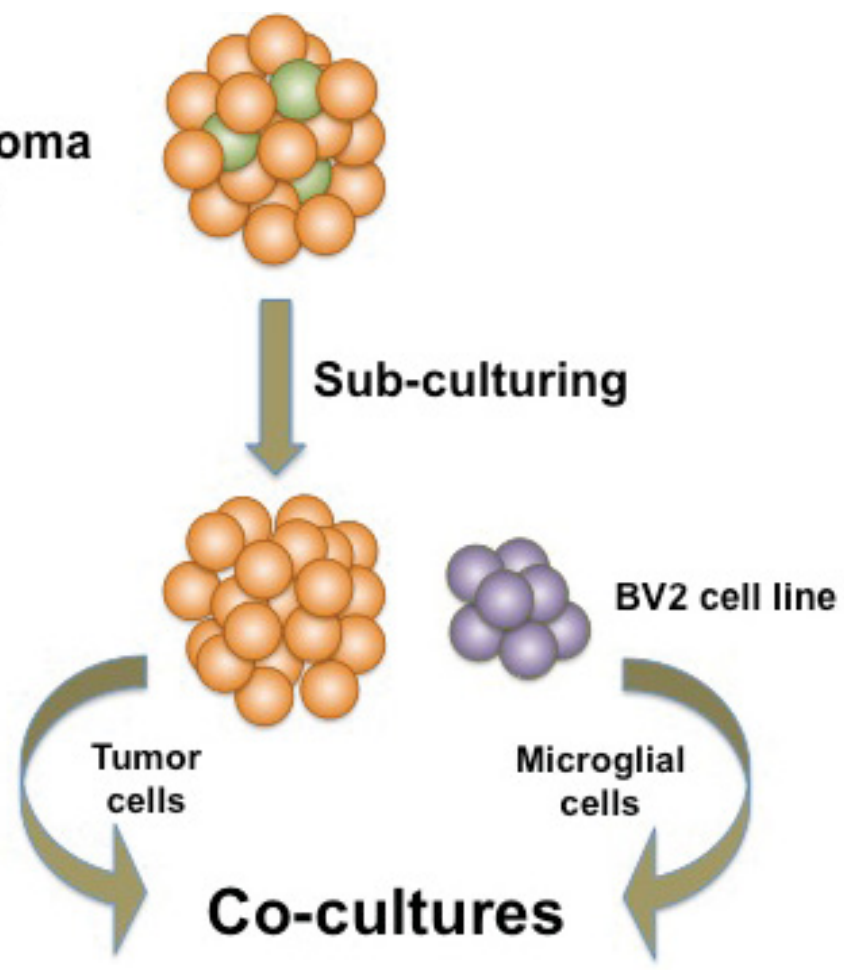

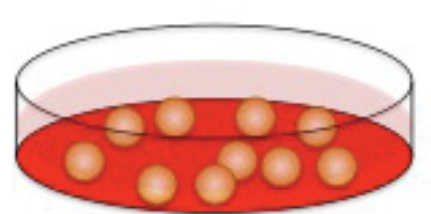

$0 \%$ microglia

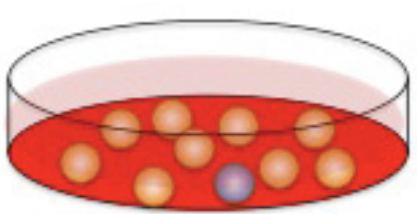

$5 \%$ microglia

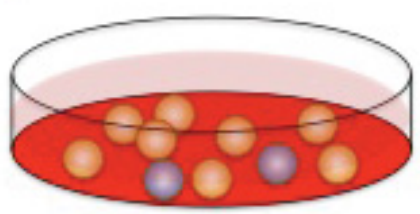

$10 \%$ microglia

Figure 2-2. A cartoon illustrating tumor-microglia co-cultures.

Primary glioma cultures were passaged a few times in order to deplete microglial cells. ICC was performed to confirm the depletion of microglial cells. Subsequently, BV2 microglial cells were mixed with glioma cells to create glioma co-cultures containing $0 \%$, $5 \%$ or $10 \%$ microglial cells. 
Table 2-1. Primer sequences used for RT-PCR.

\begin{tabular}{|c|c|c|c|c|}
\hline $\begin{array}{l}\text { Gene } \\
\text { symbol }\end{array}$ & Gene name & Gene ID\# & Probe & Primer sequence \\
\hline Arg1 & Arginase & NM_007482.3 & 2 & $\begin{array}{c}\text { F:GAATCTGCATGGGCAAC } \\
\text { R:GAATCCTGGTACATCTGGGAAC }\end{array}$ \\
\hline CD86 & CD86 antigen & NM_019388.3 & 107 & $\begin{array}{l}\text { F:GAAGCCGAATCAGCCTAGC } \\
\text { R:CAGCGTTACTATCCCGCTCT }\end{array}$ \\
\hline CD204 & $\begin{array}{l}\text { Macrophage scavenger } \\
\text { receptor } 1\end{array}$ & BC003814 & 26 & $\begin{array}{c}\text { F:GGGAGTGTAGGCGGATCA } \\
\text { R:GGAGATGATAGTAGGGTGCTCTG }\end{array}$ \\
\hline IL-1 ra & $\begin{array}{c}\text { Interleukin } 1 \text { receptor } \\
\text { antagonist }\end{array}$ & BC042532 & 34 & $\begin{array}{l}\text { F:GGCAGTGGAAGACCTTGTGT } \\
\text { R:CATCTTGCAGGGTCTTTTCC }\end{array}$ \\
\hline MMP9 & $\begin{array}{c}\text { Matrix } \\
\text { metallopeptidase } 9\end{array}$ & NM_013599.2 & 19 & $\begin{array}{l}\text { F:ACGACATAGACGGCATCCA } \\
\text { R:GCTGTGGTTCAGTTGTGGTG }\end{array}$ \\
\hline iNOS & Nitric oxide synthase 2 & NM_010927.3 & 13 & $\begin{array}{c}\text { F:CTTTGCCACGGACGAGAC } \\
\text { R:TCATTGTACTCTGAGGGCTGAC }\end{array}$ \\
\hline TGF $\beta$ & $\begin{array}{l}\text { Transforming growth } \\
\text { factor, beta } 1\end{array}$ & NM_011577.1 & 72 & $\begin{array}{c}\text { F:TGGAGCAACATGTGGAACTC } \\
\text { R:GTCAGCAGCCGGTTACCA }\end{array}$ \\
\hline $\mathrm{TNF} \alpha$ & Tumor necrosis factor & NM_013693.2 & 25 & $\begin{array}{c}\text { F:CTGTAGCCCACGTCGTAGC } \\
\text { R:TTGAGATCCATGCCGTTG }\end{array}$ \\
\hline IL-1b & Interleukin 1 beta & NM_008361.3 & 38 & $\begin{array}{l}\text { F:AGTTGACGGACCCCAAAAG } \\
\text { R:AGCTGGATGCTCTCATCAGG }\end{array}$ \\
\hline
\end{tabular}




\section{Statistical Analysis}

Mann-Whitney $U$ test was used to compare between two groups. Significance was declared when $\mathrm{p}<0.05$. All statistical analyses were performed using Statistica 8 (StatSoft Inc., Tusla, OK).

\section{Results}

\section{Generation of PDGF Driven Mouse Model of Glioma}

In the current study we used a mouse model of glioma that is driven by PDGF overexpression in neural stem cells. First, DF-1 cells were infected with RCAS PDGF or GFP vectors. Fluorescent images of DF-1/GFP cells showed nearly $100 \%$ transfection efficiency of the cells (Figure 2-3A). Virus producing cells, DF-1/PDGF, were injected intracranially in Ink4a/Arf ${ }^{-/} / \mathrm{Ntva}^{+}$mice. The developed gliomas closely represent the pathological features of human GBM such as vascular proliferation and the formation of pseudopalisading necrosis structures as described previously (Figure 2-3B) [81].

\section{Quantification of Cell Proliferation and Cell Death in Dex Treated Glioma-Bearing Mouse}

The precise effect of Dex on tumor cell proliferation remains unclear. Some studies showed that Dex decreases cell proliferation, while others showed that Dex can stimulate cell growth in vitro at low doses and is cytotoxic at high concentrations $[35,36,77]$. To address this question, paraffin embedded glioma sections from mice treated with 0 or $10 \mathrm{mg} / \mathrm{kg}$ Dex were subjected to IHC analysis. Anti-PCNA (Figure 2-4A) and anti-Ki67 (Figure 2-4B) primary antibodies were used to specifically quantify proliferating cells. We found that there was a significant decrease of both markers in Dex treated animals $(p=0.03$ and $p=0.045$ respectively). Then we examined cell death induced by Dex treatment via quantification of caspase 3, as a marker of apoptosis, in tumor sections. We observed low levels caspase- 3 staining in both treated and control tumor sections, but there was no significant difference between the two groups ( $\mathrm{p}=$ 0.521) (Figure 2-4C). As a second measurement of cell death, total area of necrosis has been quantified in tumor sections (Figure 2-4D). We found no significant difference in necrosis regions between the two groups $(p=0.902)$. To determine whether Dex decreases glioma cell proliferation, double immunofluorescence staining was performed using anti-olig2 antibody to identify tumor cells and anti-PCNA antibody as a proliferative marker. In agreement with DAB staining, there was a significant decrease in total cell proliferation in Dex treated mice $(p=0.0079)$ (Figure 2-5A). More interestingly, there was a significant decrease in proliferation in Olig2 positive cells $(\mathrm{p}=$ 0.0079) (Figure 2-5B). However, it is becoming widely accepted that tumor cell growth is greatly influenced by microglial cells $[79,86]$. Therefore, we investigated the effects of Dex on microglial cell content in tumor sections. 

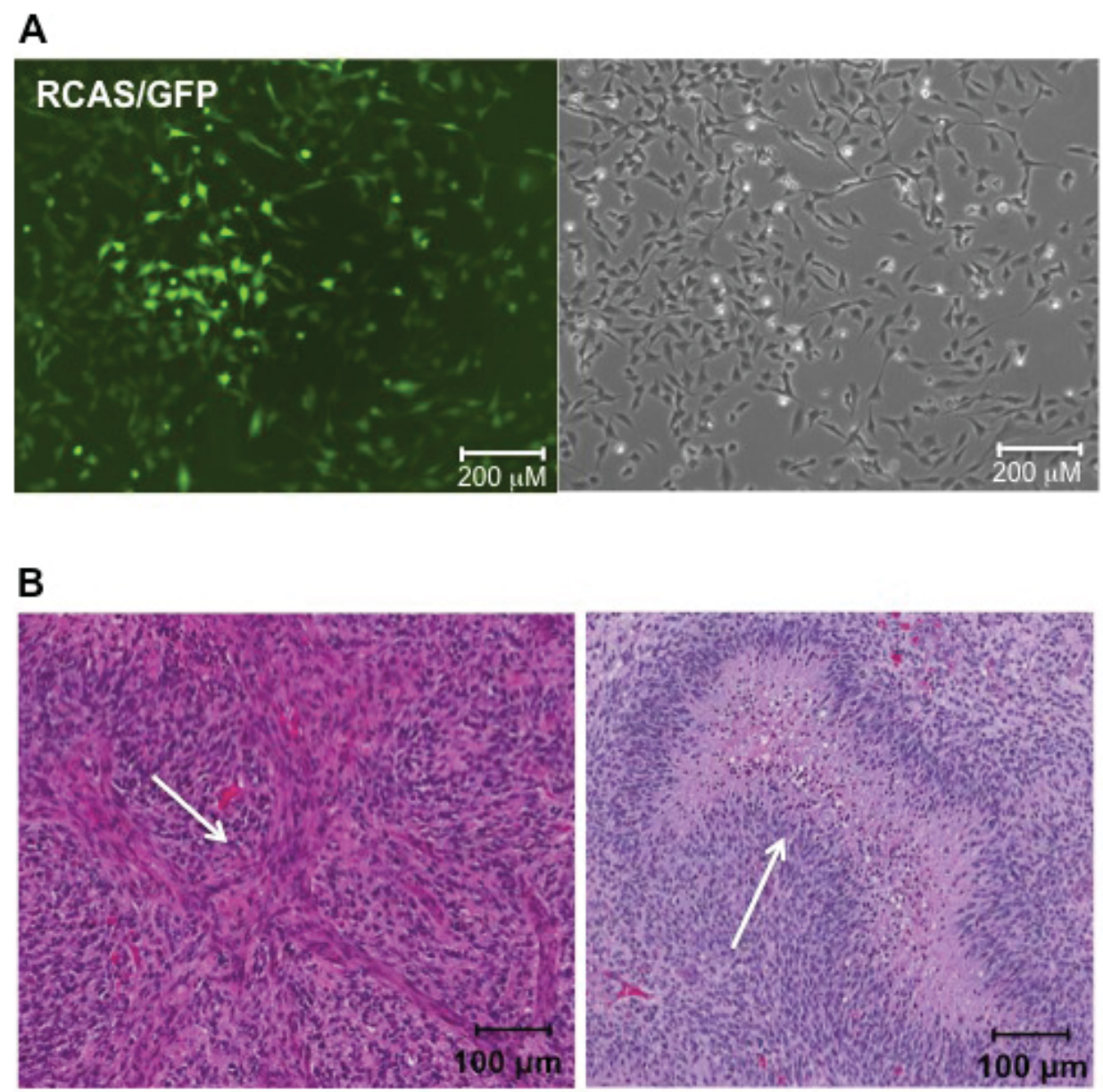

Figure 2-3. PDGF-induced mouse model of glioma.

A, DF-1 chicken fibroblast cells were infected with RCAS/PDGF or RCAS/GFP vector. DF-1/GFP cells were used to confirm transfection efficiency using Nikon eclipse TE300 fluorescent microscope $20 \mathrm{X}$ objective. B, H\&E staining of tumor sections showing vascular proliferation (left panel) and areas of necrosis area in the right panel (white arrows). 

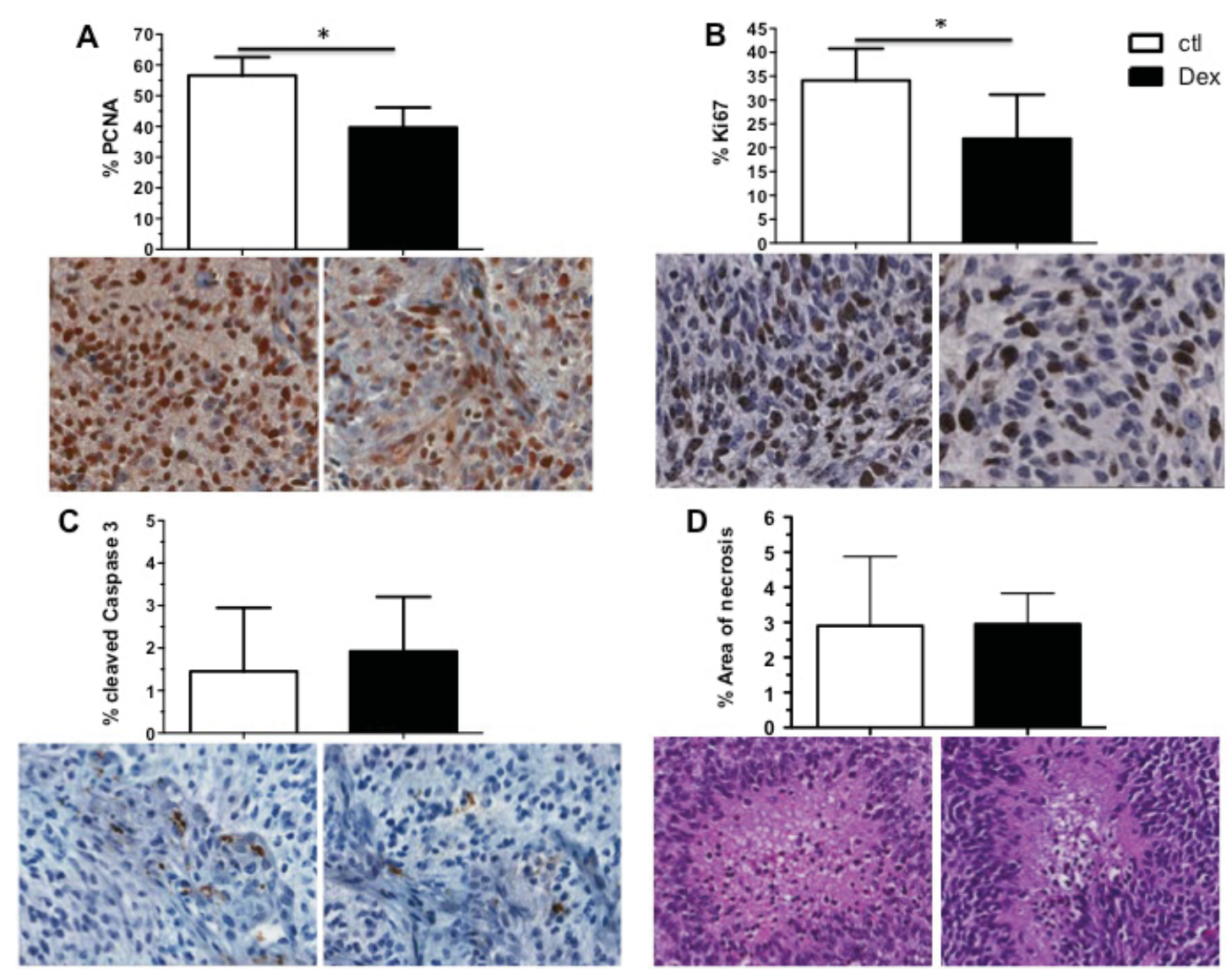

\section{Figure 2-4. IHC analysis of cell proliferation.}

A, PCNA staining was significantly reduced in Dex treated tumor sections compared to the control sections $(\mathrm{P}=0.03948) . \mathrm{B}, \mathrm{Ki} 67$ staining showed that Dex treated mice had significantly less proliferating cells compared to the controls $(\mathrm{P}=0.045) . \mathbf{C}$, Cell death was quantified using anti-cleaved caspase-3 primary antibody. The percentage of caspase-3 positive cells was very low in both treated and control tumor sections and there was not a significant difference between groups $(\mathrm{P}=0.521)$. $\mathrm{D}$, Total areas of necrosis were not significantly different between Dex treated and control mice $(\mathrm{P}=0.9015)$. Bars represent the means; whiskers represent the standard deviation $( \pm) ;{ }^{*} \mathrm{p}<0.05$. 

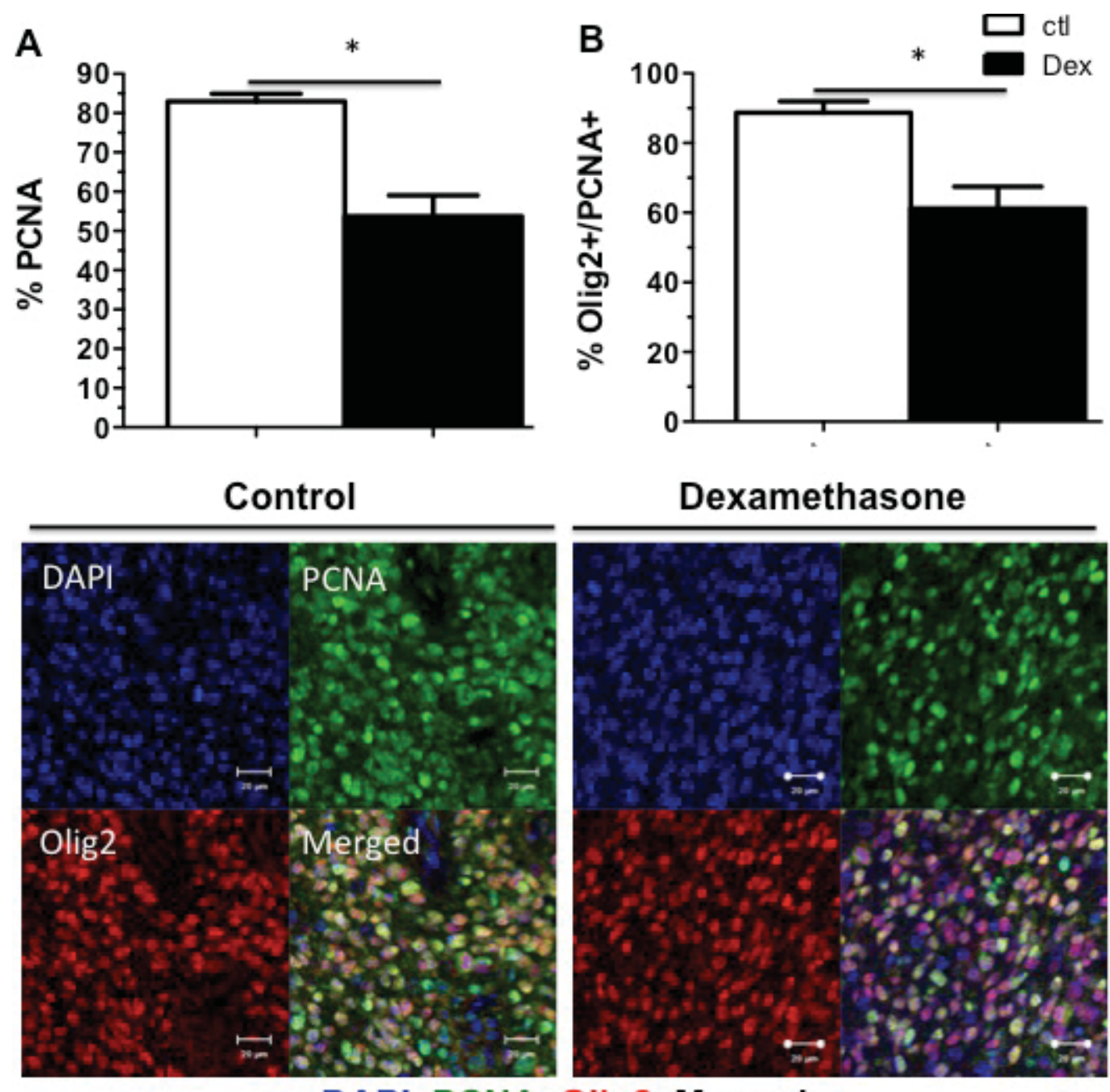

DAPI, PCNA, Olig2, Merged

Figure 2-5. IHC analysis of glioma cell proliferation.

Tumor sections were double stained with anti-Olig2 (marker of glioma cells) and PCNA (proliferative marker) primary antibodies. A, Total $\mathrm{PCNA}^{+}$cells were significantly lower in Dex treated samples compared to the controls $(\mathrm{P}=0.0079)$. $\mathbf{B}$, There was a significant decrease in the proliferation of Olig2 ${ }^{+}$cells after Dex treatment $(P=0.0079)$. Nuclei stained in blue (DAPI), PCNA stained in green (Alexa fluor 488) and Olig2 stained in red (Alexa fluor 555); (Zeiss LSM 700 Laser Scanning Microscope, 400x magnification). Bars represent the means; whiskers represent the standard deviation $( \pm){ }^{*}{ }^{*} \mathrm{p}<0.05$. 


\section{IHC Analysis of Microglial Cell Number and Proliferation Following Dex Treatment}

To determine the effect of Dex on microglial cell content in the tumor microenvironment, IHC analysis was employed to quantify microglial cells in tumor sections. Using anti-Iba-1 primary antibody to specifically stain microglia/macrophages., there was a significant reduction in Iba- $1^{+}$cells in Dex treated tumor sections when compared to the untreaded mice $(\mathrm{p}=0.0299)$ (Figure 2-6A). Next we investigated whether the decrease in microglial cell content is accompanied with a decrease in microglial cell proliferation. To test this possibility, tumor sections from both control and Dex treated mice were double stained with anti-Iba-1 and anti-PCNA primary antibodies. Indeed, there was a significant decrease in microglial proliferation in the Dex treated group when compared to the control group $(\mathrm{p}=0.0138)$ (Figure 2-6B).

\section{Gene Expression Analysis of Markers Associated with M2- and M1-Like Microglia Phenotypes}

In the tumor microenvironment, microglial cells are being reprogrammed by glioma cells to create an immunosuppressive environment as well as to promote tumor cell growth and invasion [78]. This type of activated microglia is known as M2-like microglia. In contrast M1-microglia are characterized by immune reactivity and induction of inflammation responses [78]. Therefore, we first analyzed microglial cell polarization to both phenotypes in our glioma mouse model. Total RNA was extracted from tumor samples obtained from control tumor-bearing mice using trizol method. We employed $\mathrm{Q}$ RT-PCR to assess the expression of various genes that are related to M2- (Arg1, TGF- $\beta$, CD204, MMP9, IL-1ra) and M1- (iNOS, IL-1 $\beta$, TNF $\alpha$, CD86) phenotypes. There was an obvious overexpression of TGF- $\beta$, MMP9 and Arg1 were expressed at high levels when compared to any of M1-markers that have been tested (Figure 2-7). CD204 is a cell surface marker of M2-cells and showed a similar level of expression to iNOS and higher expression compared to other M1-markers; IL-1 $\beta, \mathrm{TNF} \alpha, \mathrm{CD} 86$. In agreement with previous findings, tumor associated microglial cells exhibit on M2-like phenotype, which could promote tumor cell proliferation and progression $[79,86]$. However, we observed a decrease in tumor cell proliferation in Dex treated group, therefore we investigated whether Dex exhibits its anti-proliferation properties by modulating M2-like phenotype of microglial cells.

\section{The Effect of Dex on Microglial Cell Polarization}

We assessed gene expression of M1- and M2-signature genes in BV2 microglial cultures. The cells were grown in glioma $\mathrm{CM}$ and treated with 0 or $10 \mu \mathrm{M}$ Dex for 3 days. Total RNA was extracted using Trizol method, and Q RT-PCR was employed using $1 \mu \mathrm{g}$ of cDNA. Dex treatment resulted in a significant decrease $(\mathrm{p} \leq 0.005)$ in relative gene expression of M2-associated genes (i.e. CD204, TGF $\beta$, MMP9 and IL-1ra) when compared to the controls (Figure 2-8A). Next, we examined gene expression in vivo 

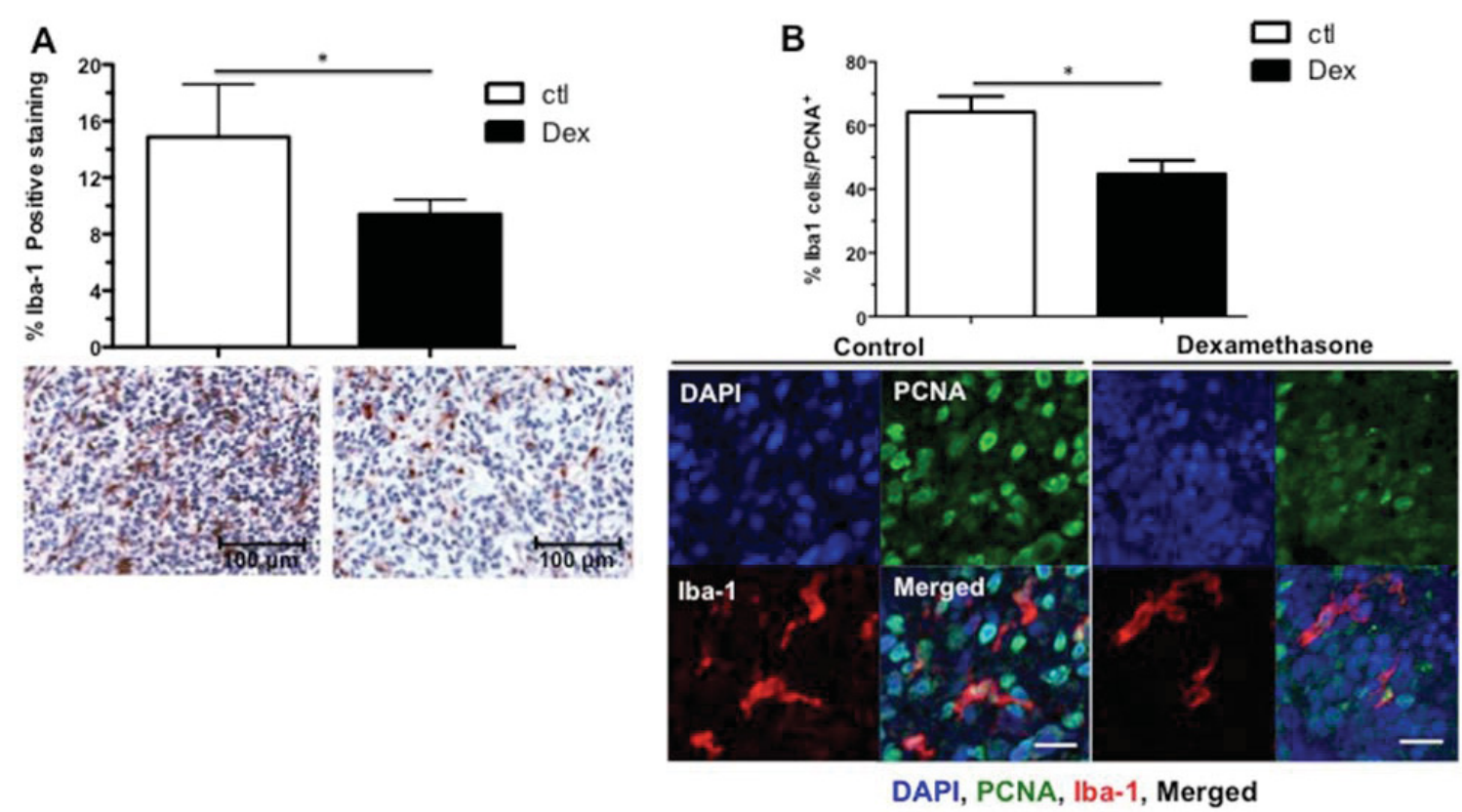

Figure 2-6. IHC analysis of microglial cell number and proliferation following Dex treatment.

A, Total number of microglial cell was quantified in paraffin embedded tumor sections stained with anti-Iba-1 primary antibody. Dex significantly reduced microglia content in vivo $(\mathrm{p}=0.0299)$. B, Tumor sections were double stained with anti-Iba-1 antibody as a marker of microglial cells and PCNA as a proliferative marker. There was a significant reduction in the proliferation of microglial cells after Dex treatment $(\mathrm{P}=0.0138)$. Nuclei stained in blue (DAPI), PCNA stained in green (Alexa fluor 488) and Iba-1 stained in red (Alexa fluor 555); nuclei stained in red (Zeiss LSM 710 Laser Scanning Microscopes, 400x magnification). Bars represent the means; whiskers represent the standard deviation $( \pm) ;{ }^{*} \mathrm{p}<0.05$. 


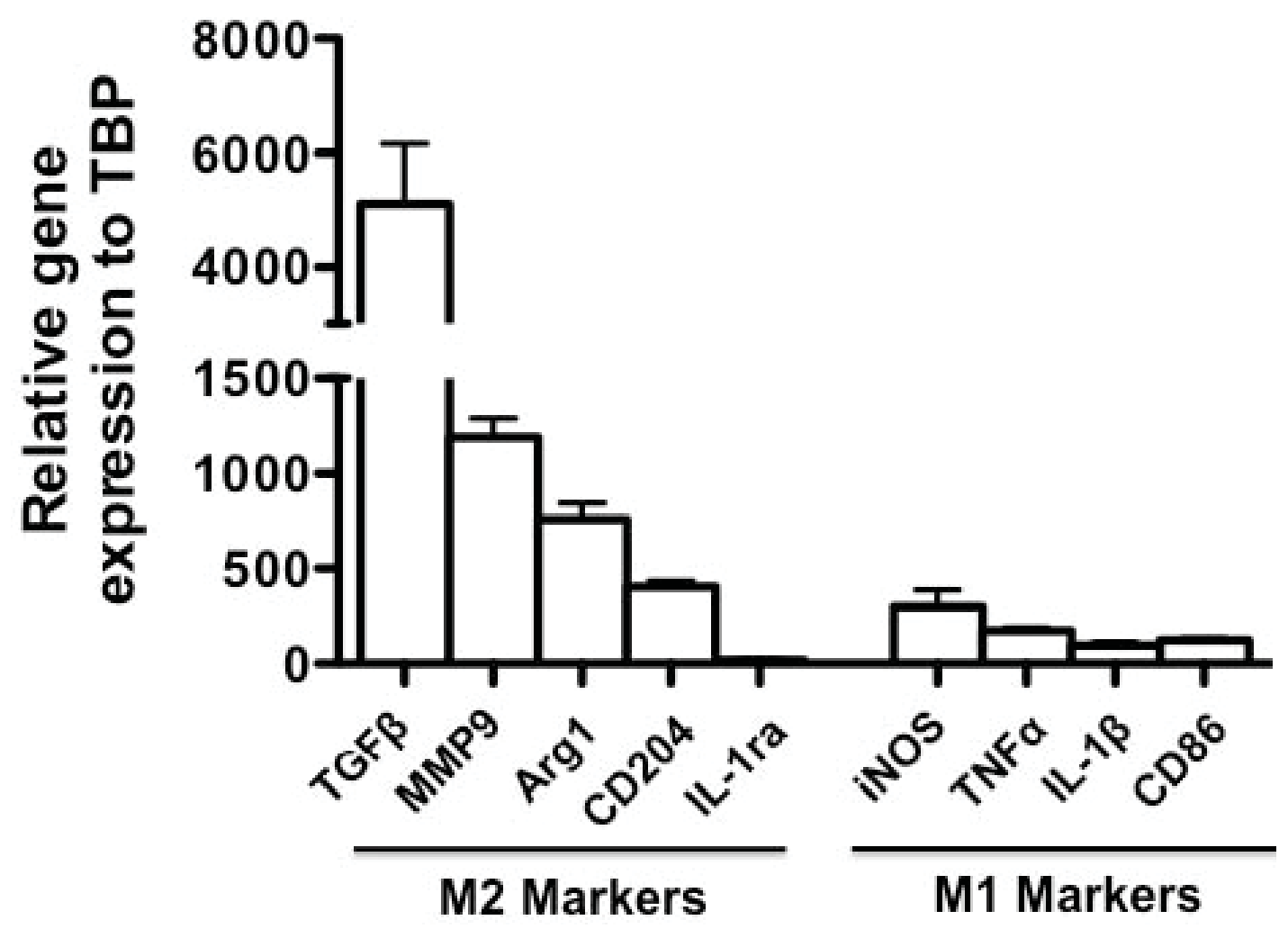

Figure 2-7. Q RT-PCR analysis of the genes associated with M1- and M2-microglial phenotypes.

Total RNA was extracted from tumor-bearing mice and Q RT-PCR was performed using Taqman probes. M2-markers of microglia (TGF $\beta$, MMP9, Arg1, and CD204) were overexpressed compared to M1-markers (iNOS, TNF $\alpha$, IL-1 $\beta$ and CD86). Bars represent the means; whiskers represent the standard deviation $( \pm)$. 
Figure 2-8. Q RT-PCR analysis of genes associated with microglial polarization in control and Dex treated tumor samples.

Total RNA was extracted from BV2 microglia cultures that were grown in glioma conditioned media and treated with 0 or $10 \mu \mathrm{M}$ Dex for 3 days. In addition, RNA was extracted from tissue samples of tumor-bearing mice treated with 0 or $10 \mathrm{mg} / \mathrm{ml}$ Dex. Q RT-PCR was employed to analyze a panel of genes associated with M2- and M1-phenotypes of microglia. A, Relative gene expression analysis showed that Dex treatment significantly decreased M2-associated genes (i.e. CD204, TGF $\beta$, MMP9, and IL-1ra) in BV2 microglial cultures. B, The same trend was observed in glioma samples. There was a decrease in MMP9, IL-1 ra and Arg1 relative expression compared to TBP. C, Dex treatment was associated with a significant decrease of all M1-associated genes (i.e. CD86, iNOS, TNF $\alpha$, IL-1 $\beta$ ) in vitro. D, However, in Dex treated animals, while there was no change in the relative expression of CD86 and iNOS, there was a significant increase in TNF $\alpha$ and IL- $1 \beta$ expression. Bars represent the means; whiskers represent the standard deviation $( \pm) ; * \mathrm{p}<0.05$. 


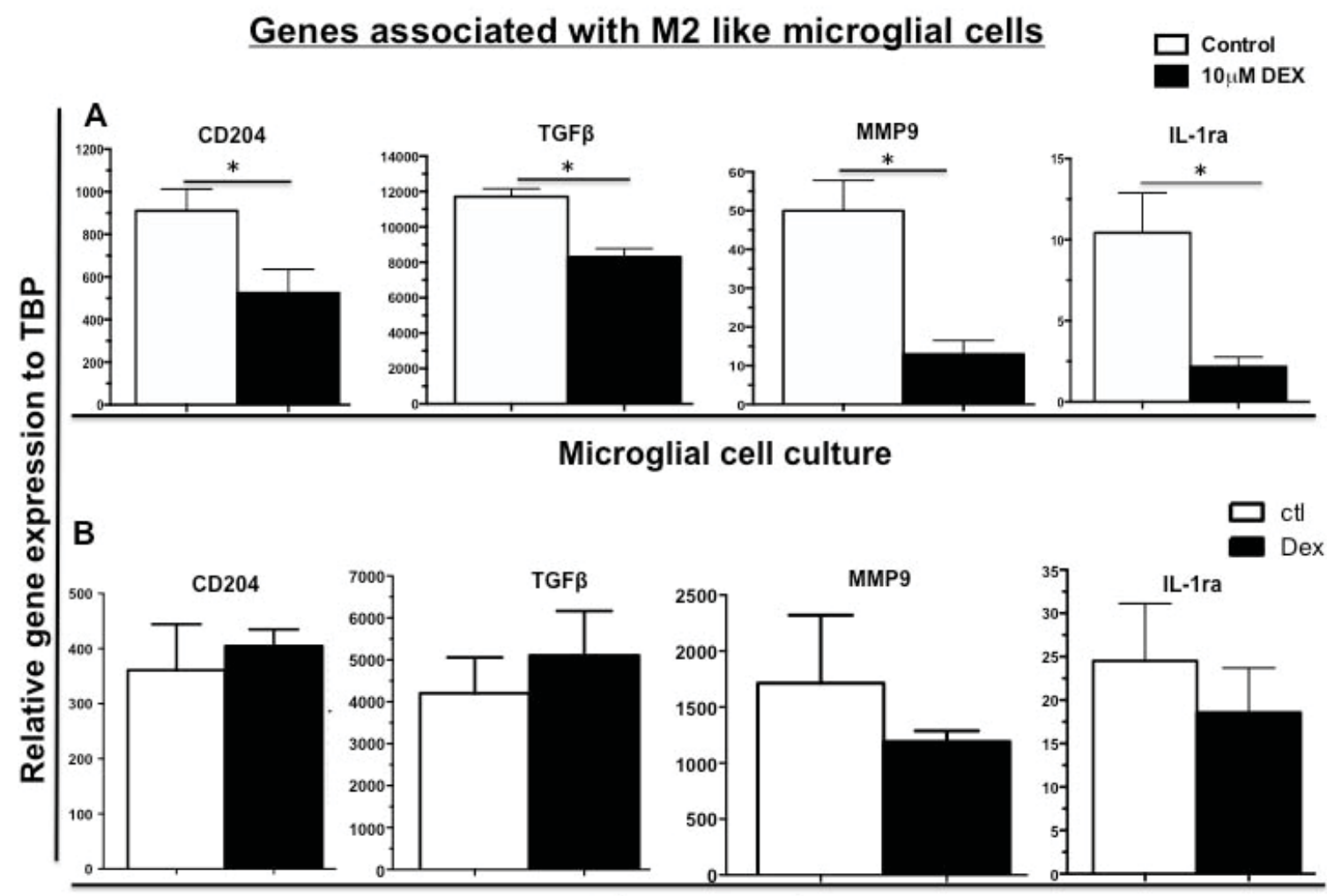

Tumor samples

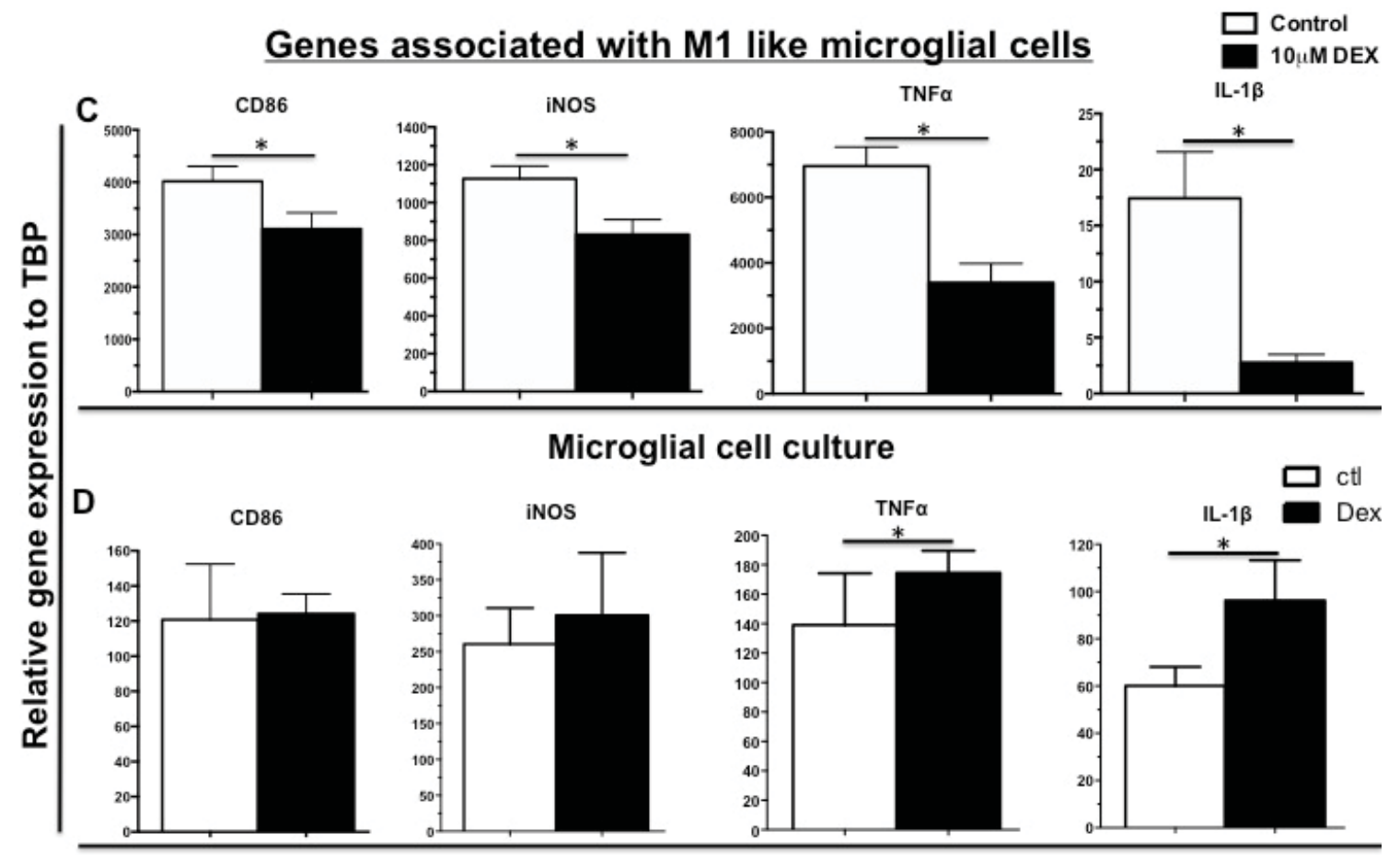

Tumor samples 
using glioma samples from control and Dex-treated mice. Consistent with the in vitro results, Dex treatment was associated with an inhibition of M2-like microglial cells. There was a modest decrease in relative gene expression of MMP9 and IL-1ra (Figure 2-8B). In BV2 cultures, the relative expression of M1-signature genes including CD86, iNOS, TNF $\alpha$ and IL-1 $\beta$ significantly was decreased in Dex treated samples $(\mathrm{p} \leq 0.005)$ (Figure 2-8C). In contrast to in vitro results, there was a significant increase in the expression of inflammatory cytokines; TNF $\alpha$ and IL-1 $\beta(\mathrm{p} \leq 0.01)$ (Figure 2-8D). The expression of CD204 or CD86 surface markers of M2- and M1-like microglial cells, respectively, was not altered in Dex treated tumor-bearing mice. Finally, we observed a slight increase in expression of TGF $\beta$ and iNOS in Dex treated mice compared to the control mice. Whether the decrease in M2-like microglial cells in tumor microenvironment could play a role in the decrease of tumor cell proliferation remains to be elucidated.

\section{In Vitro Proliferation Assessment of Primary Mixed Glioma Cultures}

Theoretically, Dex's inhibitory effect on M2, pro-tumor, microglial cells could play a role in the inhibition of tumor cell proliferation. To investigate this hypothesis, we established primary glioma cultures from control tumor-bearing mice. Glioma cultures at low passages (1-3) were used as in vitro model of mixed cultures of both tumor and stromal microglial cells. To test the ability of maintaining microglial cells in vitro, we performed ICC staining for microglia in these cultures. MTT cell viability assay was employed on glioma cultures treated with escalating doses of Dex $(0.001-100 \mu \mathrm{M})$. For all glioma cultures tested, there was no inhibitory effect of Dex (Figure 2-9A). Additionally, ICC staining showed the presence of microglial cells (6\%) (Figure 2-9B). Furthermore, cell cycle analysis was performed on glioma cultures at low passages treated with physiologically relevant Dex doses (i.e. $0,0.1,1$ and $10 \mu \mathrm{M}$ ) for 3 days by flow cytometry based technique. Although there was an a slight increase in the percentage of G1 phase after Dex treatment, which might imply a cell cycle arrest, this subtle increase may not be biologically important (Figure 2-9C). In addition ICC staining demonstrated the presence of $10 \%$ Iba- $1^{+}$cells in this culture (Figure 2-9D). Therefore, in vitro cell proliferation assays did not indicate a change in the proliferation of mixed primary glioma cultures. To further validate our findings, we generated microglia-glioma co-cultures and compared it to pure glioma cultures to address the question of whether Dex influences tumor cell proliferation indirectly by affecting microglial cells.

\section{The Effect of Dex on Glioma-Microglia Co-Cultures}

To determine the role of microglia in the cross talk between microglia and tumor cells, co-cultures of microglia-tumor cells was generated (Figure 2-2). Primary mouse glioma cultures were sub-cultured several times in order to deplete microglial cells. ICC staining using anti-Iba-1 primary antibody was used to confirm the depletion of microglial cells (Figure 2-10A). Subsequently, BV2 microglial cells were added to the 

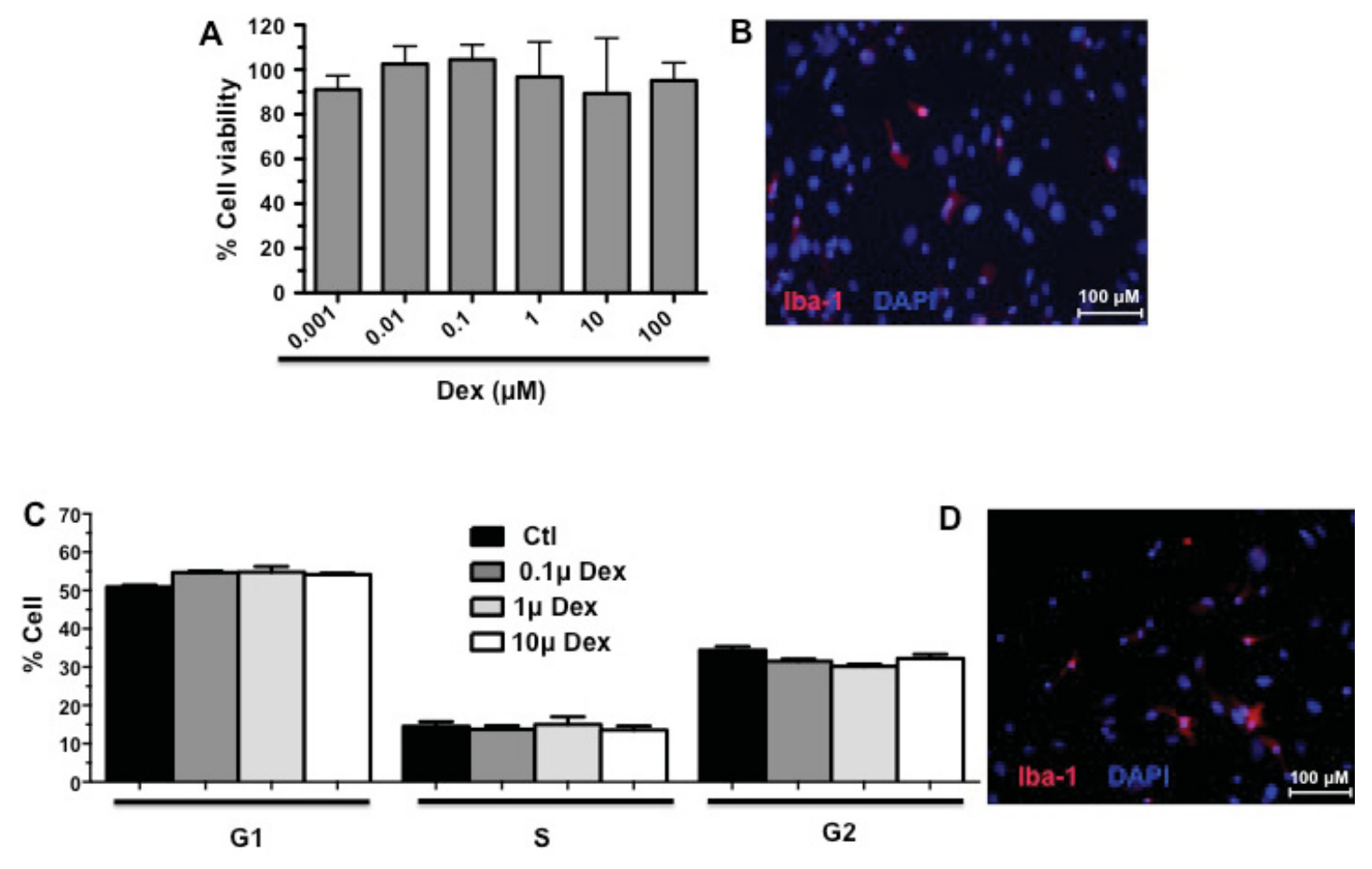

Figure 2-9. In vitro cell proliferation assays using primary mixed glioma cultures.

A, MTT assay for cell proliferation in vitro. Dex did not show an effect on cell proliferation in vitro after 3 days of incubation. B, ICC staining for Iba- $1^{+}$cells showed $6 \%$ of a microglial cells in primary mouse glioma cultures. C, Cell cycle analysis was performed on glioma cultures treated with $0.1,1$ and $10 \mu \mathrm{M}$ of Dex for 3 days. Although there was an increase in G1 phase and a subsequent decrease in G2 phase, the observed difference was not considered to be biologically significant. D, ICC staining for Iba- $1^{+}$ cells showed $10 \%$ of a microglial cells in primary mouse glioma cultures. Bars represent the means; whiskers represent the standard deviation $( \pm)$. 
Figure 2-10. The effect of Dex on glioma-microglia co-cultures.

A, ICC staining for Iba- $1^{+}$cells showed a microglial cells depletion in primary mouse glioma cultures. B, Glioma and microglial single cultures and co-cultures were plated in 96-well plates and treated with escalated doses of Dex for 3 days. There was not decrease in cell viability under any of the conditions tested. $\mathbf{C}$, Glioma and microglial single cultures, and co-cultures were subjected to cell cycle analysis assay to assess the effect of Dex on cell proliferation. We have not observed any Dex induced cell death (Sub-G1) or cell cycle arrest at either G1 or G2 phase of the cell cycle. Bars represent the means; whiskers represent the standard deviation $( \pm)$. 

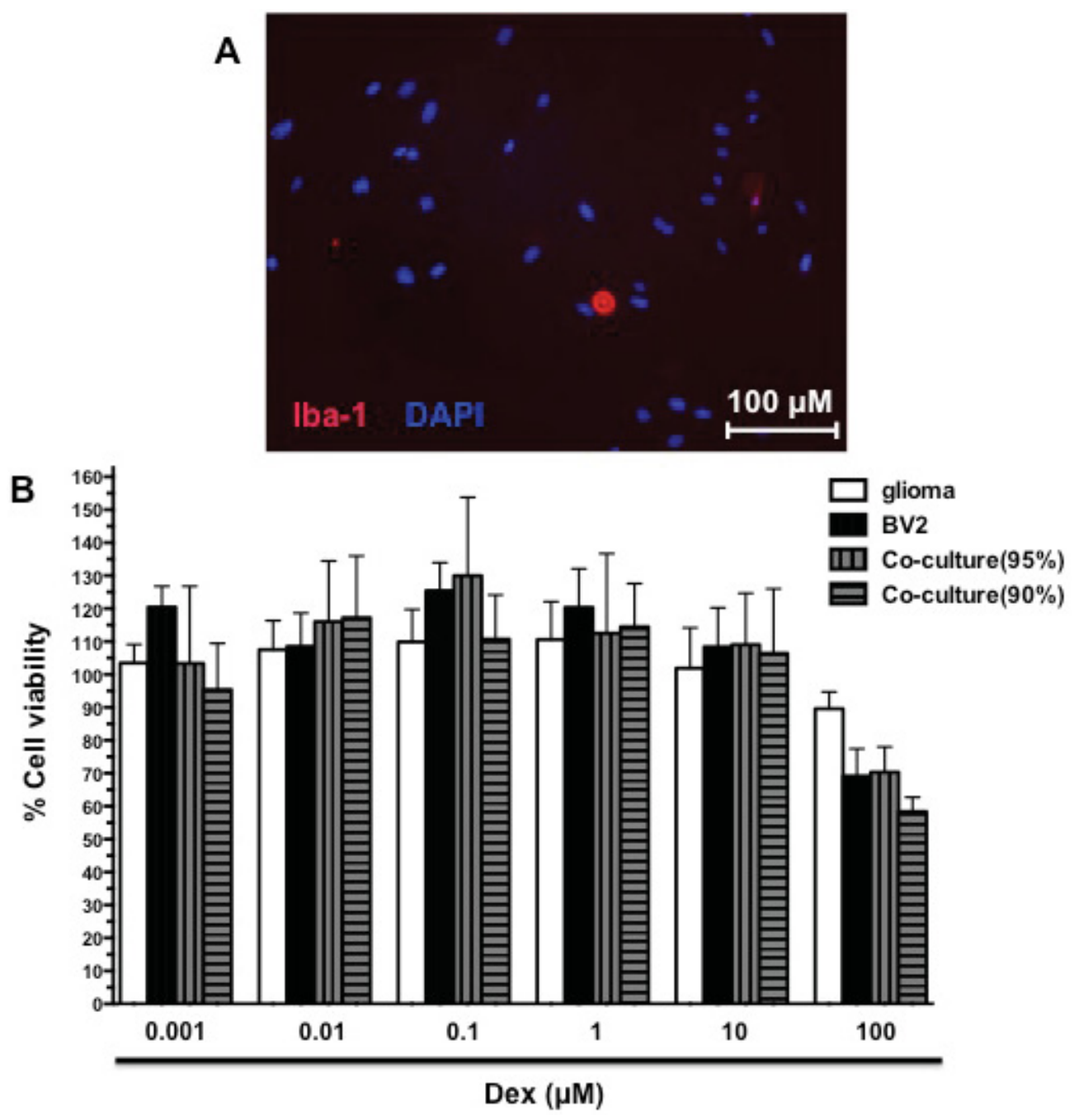

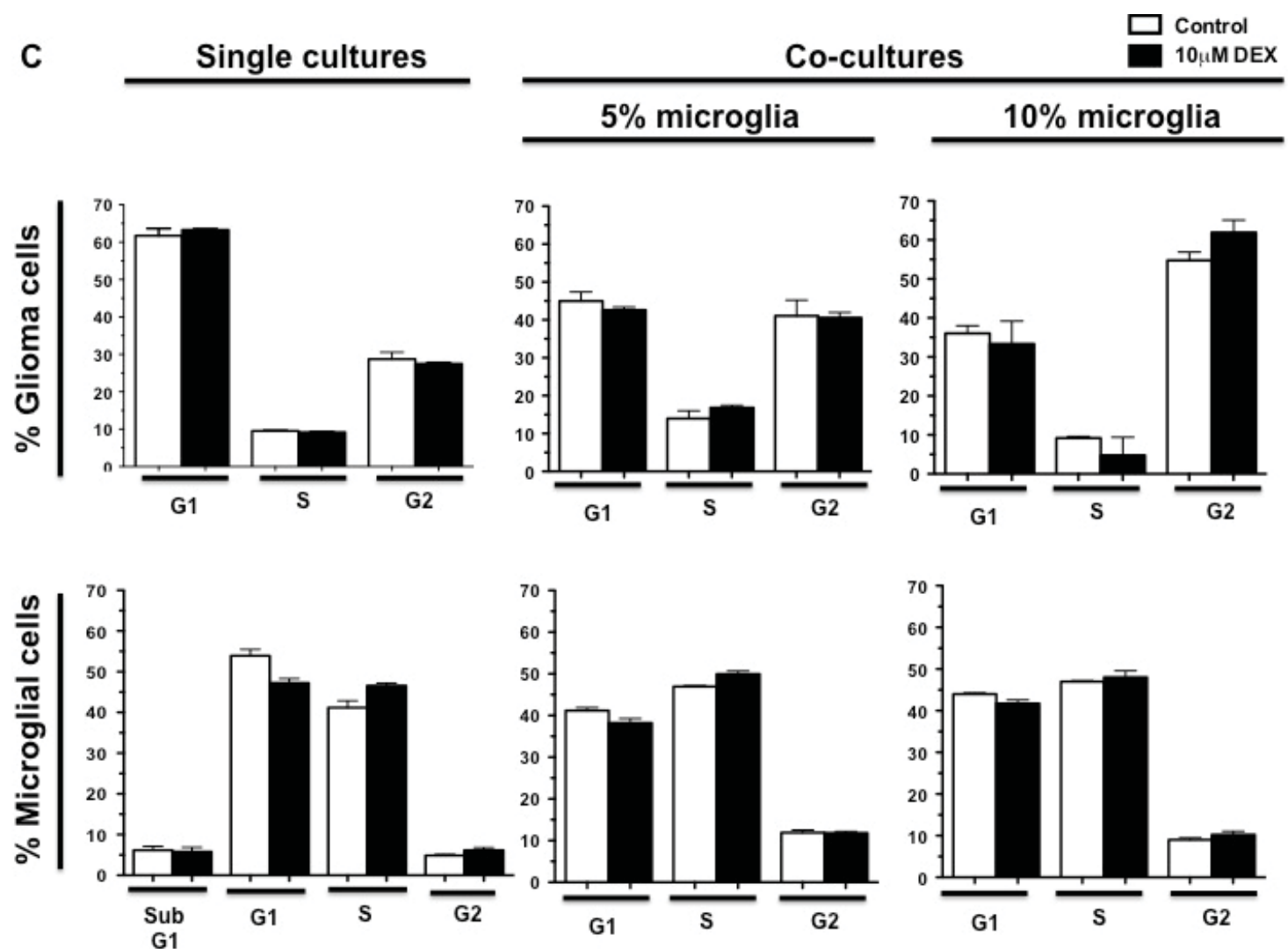

Figure 2-10. Continued. 
glioma cultures at $0 \%, 5 \%$ or $10 \%$ and seeded in 96 -well plates for MTT assay or T75 for cell cycle assay. Single cultures of glioma and BV2 cells were used as controls. After overnight incubation to allow cell attachment, the cultures were treated with Dex for 3 days. The percentage of cell viability of all the cultures did not decrease when treated with $0.001-10 \mu \mathrm{M}$ Dex. Only at $100 \mu \mathrm{M}$, Dex was able to reduce cell viability of microglia and co-cultures. However, $100 \mu \mathrm{M}$ is not a physiologically relevant dose of Dex (Figure 2-10B). Quantification of various phases of cell cycle did not reveal a dramatic change in glioma cell proliferation either in single cultures or co-cultures with microglial cells. However, we observed a subtle increase, in the G2/M phase after Dex treatment in glioma cells co-cultured with 10\% microglial cell (Figure 2-10C). This might indicate a modest cell cycle arrest at G2 phase of the cell cycle. However, Dex significantly increased microglial cell proliferation when not co-cultured as well as 5\% co-culture as shown by decreasing G0/G1 phase and increasing S phase. This effect has not been observed in MTT assay or in vivo results, which emphasize the importance of the native microenvironment to the tumor cell responsiveness.

\section{Discussion}

GBM is one of the most devastating types of cancer, because it has a very poor prognosis. For the past 10 years, the overall survival has not improved, with estimated survival of 3 years for only $7 \%$ of the patients [2]. Furthermore, all aggressive gliomas develop vasogenic edema. These corticosteroids have been used to, quickly, improve neurological symptoms resulting from edema formation $[9,72]$. However, the use of corticosteroids has been associated with numerous side effects including behavioral changes, peptic ulcer, hyperglycemia, weight gain and others $[9,10]$. Despite the intensive use of Dex in the clinic, its exact effect on the biology of glioma disease is not precisely identified. This is particularly important in regard to the effect of Dex on the responsiveness of glioma to chemo- and radiotherapy. Therefore, the aim of the current study is to investigate the effects of Dex on glioma and microglial cells in a PDGF-induced glioma mouse.

\section{The Anti-Proliferation Properties of Dex}

Whether Dex has anti-proliferative or anti-neoplastic properties, or it stimulates cell growth has been a long debate in the literature [35,36,76,77]. To understand the effect of Dex on cell proliferation, we employed a mouse model that resembles the human disease. To our knowledge this is the first study that utilizes a relevant mouse model of glioma to study the action of Dex in this mouse model. Overexpressing PDGF oncogene in neural stem cells using ink4a/ $\mathrm{Arf}^{-/}$mice drives the gliomagenesis in $100 \%$ of mice, 6 week post-infection [82]. The uniqueness of this model is that it is closely mimicking the molecular and pathological features of human GBM [81]. In addition, we employed physiological doses of Dex in our studies. Therefore, in the current study mice will be treated with a clinically relevant concentration of Dex $(10 \mathrm{mg} / \mathrm{kg})$ every day for 3 days. The human equivalent dose of $10 \mathrm{mg} / \mathrm{kg}$ is $0.8 \mathrm{mg} / \mathrm{kg}$ [83]. Indeed, at the time of 
clinical presentation, patients received $16-100 \mathrm{mg} /$ day of Dex $(0.27-1.67 \mathrm{mg} / \mathrm{kg})$ $[9,83,87,88]$.

In our genetically engineered mouse model, we found that Dex treatment decreases tumor cell proliferation as shown by PCNA and Ki67 IHC staining (Figure 2-4 and Figure 2-5). However, we did not observe significant levels of cell death in Dex treated samples. This indicates that Dex exhibits anti-proliferative activity in our mouse model. This finding can be interpreted in different ways. On one hand, several studies showed a strong correlation between PCNA proliferation index and patient's survival. In particular, better prognosis and improved patient survival are correlated with low proliferative index [89-91]. However, cancer therapeutics often target rapidly dividing cells, it becomes quite possible that Dex might compromise the responsiveness of tumor cells to chemotherapy and/or radio-therapy by slowing down tumor cell replication. Indeed, the use of corticosteroids was shown to increase tumor's radioresistance and chemoresistance [37,92,93]. GBM and fibroblast cell lines did not acquire resistance to ionizing radiation, whereas some of the carcinoma cell lines did [37,94]. Upregulation of steroids receptors following ionizing radiation therapy may be a potential mechanism responsible for Dex-induced radioresistance [38]. Such findings highlight the desperate need to understand the exact effect of Dex on tumor cells especially for patients undergoing chemotherapy or radiotherapy concurrently with corticosteroids.

The mechanism of how Dex alleviates cerebral edema is mostly unknown. However, it has been proposed that Dex might improve peritumoral edema through its anti-inflammatory effect or by inhibiting microglia cells [68,73]. Microglial cells are the dominant immune cells in the brain, comprising 5-20\% of total glial cells and up to approximately $35 \%$ in the glioma $[95,96]$. In the current study, we found that Dex decreases microglial cell content in tumor sections as confirmed by anti-Iba-1 primary antibody (Figure 2-6A). Perhaps this decrease in microglial cell content is due to slowing the proliferation rate (Figure 2-6B). However, two different phenotypes of microglial cells exists; M1 and M2. M1-like microglial cells are known to possess pro-inflammatory properties. On the other hand, various studies suggested that tumor-associated microglia could support the tumor proliferation and growth, and possess an M2-like anti-inflammatory phenotype [78,97,98].

\section{Tumor Associated Microglial Cells Possess M2-Like Phenotype}

In agreement with previous findings, we found that tumor associated microglia exhibit an M2-phenotype in PDGF-induced gliomas (Figure 2-7). Assessment of M1- and M2-signature gene expression reveals a shift in the microglia phenotype towards less M2-like (MMP9 and IL-1ra) in both in vitro and in vivo samples upon Dex treatment (Figure 2-8A). On the other hand, the expression of M1-associated genes was inconsistent when in vitro data was compared to in vivo gene expression (Figure 2-8B) raising the concern of the reliability of the in vitro models. We observed a decrease in the expression of IL-1ra and Arg1, anti-inflammatory genes, in Dex treated tumor samples. Of notice, IL-1 ra is an antagonist to IL- $1 \beta$ and Arinase 1 competes with and iNOS 
substrate $[99,100]$. When this finding is combined with the observed modest increase in TNF $\alpha$ and IL-1 $\beta$ pro-inflammatory cytokines, it becomes quite obvious that Dex might not relieve the edema through its anti-inflammatory effect. However, our study was conducted after 3 days of Dex treatment, it would be very interesting to investigate the effect of Dex on microglial cell polarization over a prolonged period of time.

\section{Dex Decreases Microglial Cell Polarization to M2-Phenotype}

Interestingly, Dex treatment was associated with a decrease in MMP9 expression, possibly leading to suppression of glioma invasion. MMP9 is a member of matrix metalloproteinases family, which is produced primarily by immune cells such as macrophages/microglias and neutrophils [101]. MMP9 plays a pivotal role in tumor progression and invasion [97]. MMP9 deficient mice had less lung metastases in both melanoma and lung carcinoma animal models [102]. More importantly, MMP9 plays an important role in angiogenesis and induction of cell proliferation [101,103,104]. These findings raise the question of whether this decrease in M2-pro-tumor phenotype could play a role in tumor cell proliferation in Dex treated samples. Therefore, we investigated the anti-proliferative effect of Dex using low passage glioma cultures containing both tumor and microglial cells. Quite unexpectedly, we could not detect a cell proliferation inhibition following Dex treatment (Figure 2-9). We tested several cultures that contain variable percentages of microglial cell. However an anti-proliferative effect of Dex was not detected. To further elucidate the potential role of microglia in tumor cell proliferation, we established glioma-microglial cells cultures and examined glioma cell proliferation in Dex treated culture with and without microglial cells. However, we could not detect a decrease in the proliferative capacity of either glioma nor microglial cells as single or co-cultures. On the other hand, our in vivo data confirm a decrease in proliferation of both cell types following Dex treatment. These contradicting findings highlight the difference between in vitro and in vivo conditions and this does not eliminate the possibility that microglial cell play an important role in supporting tumor cell proliferation.

In conclusion, Dex treatment is associated with a decrease in tumor and microglial cell proliferation in PDGF-driven glioma samples. Furthermore, pro-tumor microglial phenotype, M2 seems to decrease after Dex treatment. Taken together, Dex might act against glioma favorable conditions in our mouse model. However, this effect might or might not be recapitulated when Dex is combined with DNA damaging agents such as ionizing radiation. The current study shows the importance of studying the exact mechanism of action of Dex in the context of glioma. 


\title{
CHAPTER 3. THIOPURINE-INDUCED TOXICITY IN ASTROCYTES IS DEPENDENT ON TPMT ACTIVITY*
}

\author{
Introduction
}

\section{TPMT History and Discovery}

Thiopurine methyltransferase (TPMT) has been studied extensively because of its critical role in the methylation of thiopurine agents and their subsequent metabolites. Primarily, this biotransformation leads to the inactivation of these agents. Thiopurine drugs, such as thioguanine (TG), mercaptopurine (MP) and azathiopurine (AZA) are widely used as anticancer and anti-inflammatory agents and as immunosuppressants. However, since the use of these agents has been linked with the development of secondary brain tumors in TPMT-deficient patients, it is pivotal to understand the toxicity effects of thiopurines in the brain $[42,47]$.

The activity of partially purified human TPMT was studied for the first time in 1978 [105] and the first application of activity measurements was done in 1980 [106]. These studies raised the possibility that human RBC TPMT activity is inherited and TPMT genetic polymorphism is contributing to inter-individual differences in the metabolism of thiopurines. It was not until 1995 that the first inactive TPMT allele had been identified [107]. TPMT is found in the cytoplasm of eukaryotes and prokaryotes [108]. It catalyzes S-methylation of aromatic and heterocyclic compounds (including thiopurines) using S-adenosyl-L-methionine [41] however, the endogenous substrate for TPMT is yet to be identified. Human TPMT gene has 10 exon and is located in chromosome $6 \mathrm{p} 22.3[109,110]$. The gene encodes a $28 \mathrm{kDA}$ protein and is comprised of 245 amino acids [111].

\section{Metabolism of Thiopurine Drugs}

Thiopurines are pro-drugs that must be metabolized intracellularly by hypoxanthine phosphoribosyl transferase (HPRT) to TG nucleotides (TGN) to exert their cytotoxic effect [39]. First, AZA can be converted non-enzymatically to MP, which is then converted to thioinosine monophospahte (TIMP) by HPRT (Figure 3-1). The same enzyme catalyzes the conversion on TG to thioguanosine monophospahte (TGMP). Thioxanthiosine monophosphate (TXMP) and inositol monophosphate dehydrogenase (IMPDH) catalyze the conversion of TIMP to TGMP, which is subsequently converted to TGN. TGN is a good substrate for DNA polymerases and hence is incorporated into

\footnotetext{
*Adapted with permission from Hosni-Ahmed A, Barnes JD, Wan J, Jones TS (2011) Thiopurine methyltransferase predicts the extent of cytotoxicty and DNA damage in astroglial cells after thioguanine exposure. PLoS ONE 6: e29163.
} 


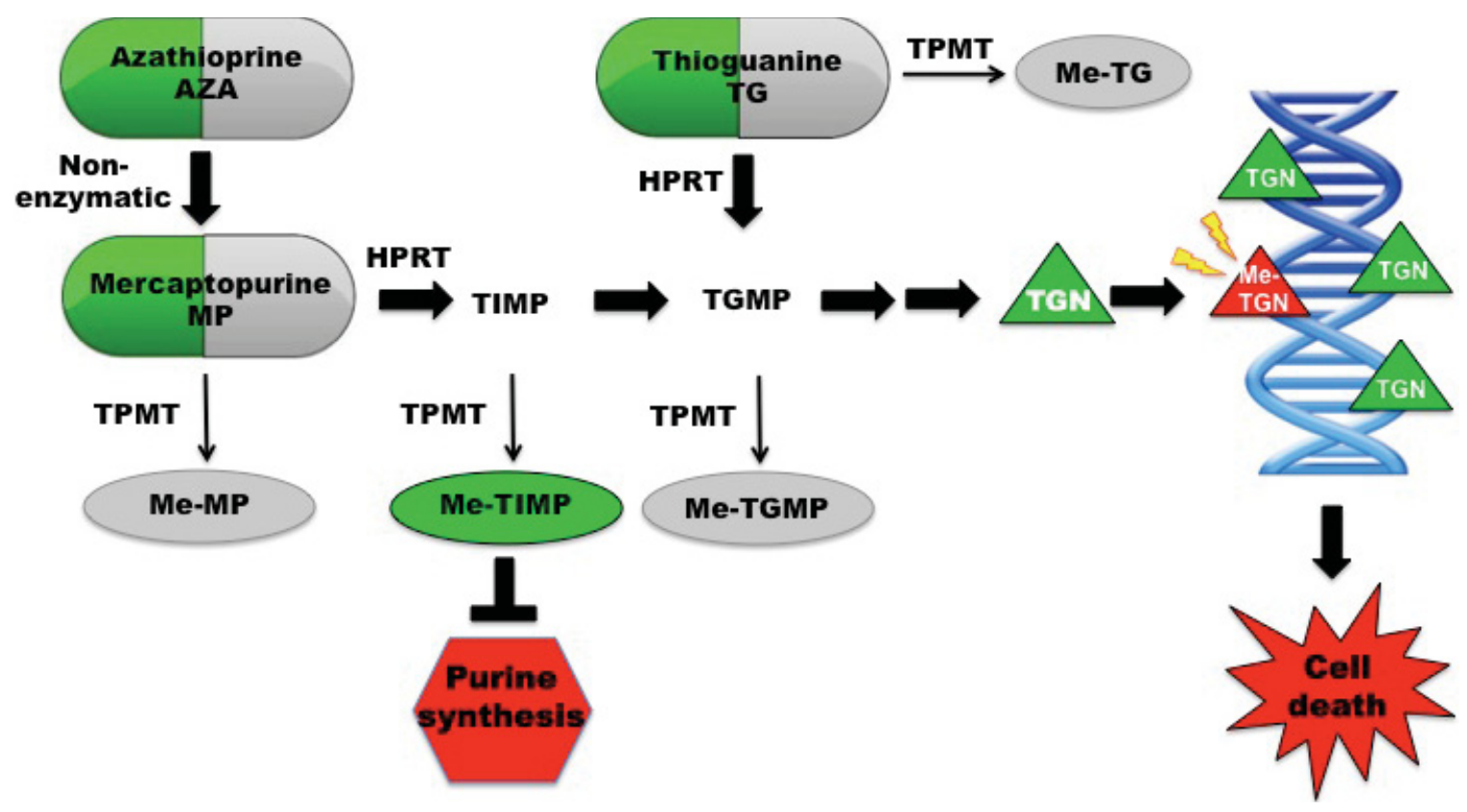

Figure 3-1. Thiopurine drug metabolism pathway.

Azathioprine (AZA) is a prodrug of mercaptopurine (MP). Thioguanine (TG) and MP can be converted by hypoxanthine phosphoribosyl transferase (HPRT) to thioguanine nucleotide (TGNs) metabolites. MP can also be converted to the methylthioinosine monophosphate (meTIMP) by thiopurine methyltransferase (TPMT), which is purine synthesis inhibitor; however, thioguanine bypasses the conversion to this metabolite. Thiopurines can be converted to inactive metabolites [i.e. methyl-mercaptopurine (meMP); methyl-thioguanine (meTG); and methyl-thioguanine nucleotides (meTGNs)] by TPMT. TGN metabolites can be incorporated into DNA and RNA leading to cell death. However, DNA incorporation is believed to be the primary mode of cytotoxicity. 
DNA. At steady state levels, approximately $10^{5}$ to $10^{6}$ TGN incorporates into DNA per cell $[112,113]$. However this level of DNA incorporation is tolerated by the cell and further modification, via non enzymatic methylation, of TGN (me-TGN) is required for its toxic effects. Me-TGN could induce single strand breaks in the DNA, interstrand cross links and sister chromatid exchange [114-117]. On the other hand, thiopurine agents and their metabolites can be inactivated by xanthine oxidase and, more importantly, TPMT into thiopuric acid and 6-methyl derivatives respectively. Therefore, TPMT activity is inversely correlated with TGN and toxicity levels [118].

\section{TPMT Genotypes}

The heterogeneity in TPMT activity is predominantly due to single nucleotide polymorphisms (SNPs). Despite the fact that 29 TPMT variant alleles have been identified [119], TPMT*2, TPMT*3A, TPMT*3B and TPMT*3C variants account for approximately $90 \%$ of cases with reduced enzymatic activity [43,120-124]. TPMT*2, the first variant allele discovered, consists of G238C SNP which results in Ala80Pro amino acid substitution [107]. This substitution leads to the distortion of the tertiary structure of the TPMT protein and the loss of catalytic activity. However, the most clinically relevant mutant allele is TPMT*3A which is characterized by two SNPs; G460A at exon 7 and A719G at exon 10. These two SNPs result in Ala154Thr and Tyr240Cys amino acids substitutions. Heterologous expression of human TPMT*2, TPMT*3A in yeast showed enhanced proteolysis with a 15 min half live of the two mutant variants as compared with $18 \mathrm{hr}$ for the wild-type TPMT [43]. When expressed in mammalian cells, TPMT*3B and TPMT*3C variants, showed an enhanced degradation rate [125]. They consist of individual Ala154Thr and Tyr240Cys SNPs, respectively.

The frequency of mutant TPMT variants differs among various ethnic populations. For example, TPMT*3A is the most prevalent variant allele in American Caucasian, Germans, Italians and Jordanians [126-129]. In addition, TPMT*3A is the only detected variant allele in West Asia population [130]. However, TPMT*3A is predominant in African-Americans, Egyptians and Ghanaians [126,131,132].

Although TPMT genotypes are a good predictor of TPMT activity, non-genetic factors might also influence the enzyme activity. For example, thiopurines have been shown to induce TPMT activity [133]. On the other hand aspirin and sulfasalazine are potent inhibitors of TPMT when co-administered with thiopurines [134].

\section{TPMT Phenotypes}

TPMT activity is the best predictor of thiopurine efficacy and toxicity. TPMT deficient patients are at high risk of life threatening hematopoietic toxicity when treated with a standard dose of thiopurine drugs, due to accumulation of TGNs at high levels [44-46,135-137]. Weinshilboum and Sladek [106] reported a trimodal distribution in TPMT activity. The level of TPMT protein expression is directly correlated TPMT 
protein activity. Approximately $90 \%$ of the population are homozygous for wild-type (WT) TPMT alleles and have high TPMT activity, about $10 \%$ are hetrozygous with intermediate protein activity and $\sim 0.3 \%$ are homozygous for variant alleles, and lack detectable enzyme activity. Enhanced TPMT protein degradation, as compared to the WT TPMT, has been shown to be the mechanism that is responsible for low TPMT activity caused by variant alleles [43].

\section{TPMT Importance in the Clinical Settings}

Thiopurines are antimetabolites that have been used as anti-cancer agents and as immunosuppressants. Since the 1950s, thioguanine and mercaptopurine have been used to treat childhood acute lymphoblastic leukemia (ALL) $[55,56]$. Several studies have indicated inter-patient variability in TGN concentrations [137-140]. TPMT polymorphisms and differences in 6-MP absorption could in part explain inter-patient variability $[46,118,141,142]$. With regard to treatment outcome in children with ALL, high TGN concentrations have been associated with a better relapse-free survival compared to patients with lower TGN concentrations [137,143]. TPMT phenotype is a good predictor for thiopurine efficacy and toxicity. For example, a better outcome is associated with lower TPMT activity [41]. In general, mutant homozygous or heterozygous patients with low TPMT activity should be treated with lower thiopurine doses to avoid toxicity. Several studies have shown that TPMT deficient patients treated with 6-MP and receiving cranial irradiation have a high incidence of secondary brain tumors [47].

Azathioprine and 6-mercaptopurine are widely used to treat inflammatory bowel disease. It has been shown that TPMT SNPs are associated with thiopurine-induced adverse drug reactions and bone marrow toxicity but not hepatotoxicity or pancreatitis [144]. In the same line of evidence, TPMT polymorphisms have been associated with azathioprine toxicity in renal transplant recipient patients [145]. TPMT is constitutively expressed in several tissues including blood, kidney, liver, and brain [146-150]. The association of TPMT status with brain tumor risk after thiopurine exposure prompts the question of whether TPMT genotypes can predict thiopurine drug phenotypes in astroglial cells. The relevance of TPMT status to thiopurine-associated phenotypes in important brain cell populations has not been studied. Indeed, the first step to address the question of whether TPMT plays any role in brain cancer risk after thiopurine treatment is to determine whether TPMT phenotypes in the brain are similar to those from other tissues. Thiopurine drug-associated genotoxicity has been linked to mutagenesis and transformative events [151-153]. We hypothesize that TPMT deficiency can lead to greater genotoxicity (i.e. DNA damage) and cytotoxicity in astroglial cells after thiopurine exposure. To address this hypothesis we used primary astrocytes isolated from transgenic mice of each TPMT genotype and performed in vitro studies to compare thiopurine-induced cytotoxicity and DNA damage between TPMT genotypes. We also used established human glioma cell lines as model astroglial cells to validate the findings observed in primary mouse astrocytes. 


\section{Methods and Materials}

\section{Animals}

The TPMT knockout mice were a generous gift of Dr. Mary Relling (St. Jude Children's Research Hospital). The $\mathrm{TPMT}^{+/}$, and $\mathrm{TPMT}^{-/-}$mice were indistinguishable from TPMT ${ }^{+/+}$mice by appearance, life span, and organ histology (i.e. brain, liver, spleen, intestine, thymus, and lymph nodes). Animals were maintained in accordance to $\mathrm{NIH}$ guidelines for the care and use of laboratory animals and were housed in an AAALAC accredited facility. The University of Tennessee Health Science Center Institutional Animal Care and Use Committee approved all animal procedures.

\section{Primary Astrocyte Cultures and Cell Lines}

Astrocyte cultures were established from 2-5 day old pups of each TPMT genotype. The DNA extracted from tail snips was used to confirm TPMT genotype. A dissecting microscope was used to remove the meninges and hippocampus, and the cortices were mechanically dissociated. Subsequently, cultures were established and maintained in Dulbecco's modified Eagle's medium/Ham's F-12 50/50 mix (Cellgro, Hemdon, VA) supplemented with 10\% fetal bovine serum, $2 \mathrm{mM}$ L-glutamine, 100 $\mathrm{IU} / \mathrm{ml}$ penicillin, $100 \mu \mathrm{g} / \mathrm{ml}$ streptomycin and $20 \mathrm{ng} / \mathrm{ml}$ epidermal growth factor (Millipore Co., Bedford, MA) and grown in Primaria flasks, (BD Bioscience, San Jose, CA). Cells were refed with $20 \mathrm{ng} / \mathrm{ml}$ epidermal growth factor after three days of incubation, and the media was changed after five days. At passage 2 three individual primary cultures of the same genotype were pooled and seeded for experiments. Only cultures with greater than $80 \%$ astrocyte purity as determined by GFAP immunohistochemistry were included in experiments.

The T98 and A172 human glioma cell lines were obtained from the American Type Culture Collection (ATCC, Manassas, VA, USA). T98 cells were grown in Minimum Essential Medium Eagle (Cellgro, Hemdon, VA), supplemented with 10\% fetal bovine serum, $2 \mathrm{mM}$ L-glutamine, $0.1 \mathrm{mM}$ nonessential amino acids, $1 \mathrm{mM}$ sodium pyruvate, $100 \mathrm{IU} / \mathrm{ml}$ penicillin, and $100 \mu \mathrm{g} / \mathrm{ml}$ streptomycin. A172 cells were grown in Dulbecco's Modified Eagle's Medium (Cellgro, Hemdon, VA), supplemented with 10\% fetal bovine serum, $2 \mathrm{mM}$ L-glutamine, $100 \mathrm{IU} / \mathrm{ml}$ penicillin, and $100 \mu \mathrm{g} / \mathrm{ml}$ streptomycin. All cell cultures were maintained at $37^{\circ} \mathrm{C}$ in a $5 \% \mathrm{CO}_{2}$ humidified atmosphere.

\section{Lentiviral Transduction}

Human TPMT cDNA was obtained by digesting pCMV6-TPMT plasmid (OriGene Technologies, Inc., Rockville, MD) at the BamHI and EcoRI sites. TPMT cDNA was then amplified by PCR, purified through agarose gel electrophoresis, and 
subcloned into BamHI and EcoRI sites of pLenti-pgk-puro vector (Viral vector core, UTHSC) (Figure 3-2). The pLenti-TPMT-pgk-puro and pLenti-GFP-pgk-puro lentivial vectors were produced in 293T cells [154]. The lentiviral vectors were used to transfect the A172 cell line in the presence of $6 \mu \mathrm{g} / \mathrm{mL}$ polybrene (Sigma-Aldrich, St. Louis, MO). Transfection efficiency was monitored by GFP expression using fluorescent microscope. The mock- (A172 $\left.{ }^{\text {mock }}\right)$ and TPMT-transfected (A172 ${ }^{\text {TPMT }}$ ) cell lines were seeded for experiments at comparable passage numbers and TPMT activity was measured prior to the experiments.

\section{Cell Proliferation Comparison}

The doubling rates for primary astrocyte cultures were determined and used as an index of cell proliferation. At passage two, 75,000 cells/well were seeded in duplicate 6-well plates. The cells from one well of each plate were harvested daily using $0.05 \%$ Trypsin-EDTA (Gibco, Life Technologies Inc., Grand Island, NY) and viable cells were counted by trypan blue. Doubling rates were calculated based on Equation 3-1. T2 = Harvesting time, $\mathrm{T} 1=$ Initial time, $\mathrm{N}=$ Final cell concentration, and $\mathrm{N}_{0}=$ Initial cell concentration. The mean doubling rate was calculated from duplicate experiments.

$$
\text { Doubling time }(\mathrm{hr})=(\mathrm{T} 2-\mathrm{T} 1) /\left[\log 2 \times\left(\log \mathrm{N}-\log \mathrm{N}_{0}\right)\right]
$$

\section{TPMT Activity Assay}

Primary mouse astrocyte and human glioma cell lines were sonicated to perform the TPMT activity assay. The level of TPMT activity was determined using a non-chelated radiochemical assay $[105,147]$ with minor modifications. TPMT activity was quantitated by measuring the conversion of 6-mercaptopurine (Sigma-Aldrich, St. Louis, MO) to radioactively labeled 6-methylmercaptopurine with [14C] S-adenosyl-L-methionine (SAM) (Perkin Elmer, Waltham, MA). The protein concentration of each lysate was measured prior to performing the assay and was used to calculate TPMT activity. The mean activities for each genotype were calculated using data from triplicate assays.

\section{MTT Assay}

Cell viability was measured using 3-(4,5-dimethylthiazol-2-yl)-2,5-diphenyl tetrazolium bromide (MTT) (Sigma-Aldrich, St. Louis, MO). In brief, 1000 cells were seeded per well in 96-well plates. Plates were pre-coated with poly-L-ornithine (Sigma-Aldrich, St. Louis, MO) and laminin (Invitrogen, Carlsbad, CA) for primary astrocytes to facilitate cell attachment. A stock solution $(15 \mathrm{mM})$ of TG (Sigma-Aldrich, St. Louis, MO) was prepared by dissolving TG in $0.1 \mathrm{~N} \mathrm{NaOH}$. Cells were allowed to attach overnight and TG or vehicle control was added to the appropriate wells. After five days, MTT was added and allowed to incubate for $3 \mathrm{hr}$. The media was carefully 


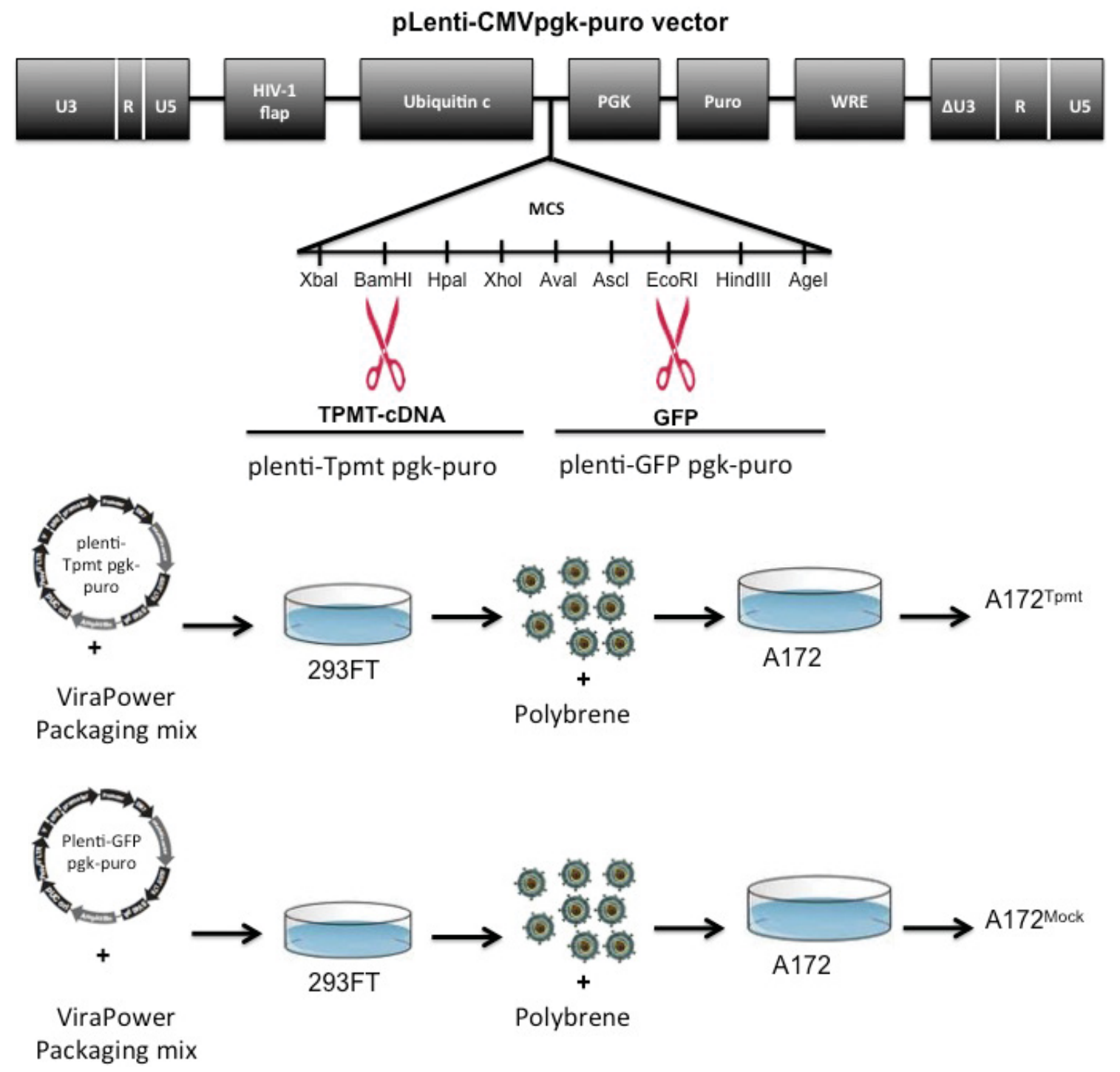

Figure 3-2. pLenti-pgk viral vector.

TPMT or GFP cDNA was subcloned into pLenti-pgk-puro vector using BamHI and EcoRI sites. The lentivial vectors were packaged in $293 \mathrm{~T}$ cells. The mock- (A172 ${ }^{\text {mock }}$ ) and TPMT-transduced (A172 ${ }^{\mathrm{TPMT}}$ ) cell lines were generated by transducing A172 cell line with either pLenti-TPMT-pgk-puro or pLenti-GFP-pgk-puro lentivial vector, respectively. 
aspirated and the purple formazan crystals were dissolved in $100 \mu 1$ DMSO (Fisher Scientific Co., Fair Lawn, NJ). Absorbance was measured using a FLx800 fluorescence microplate reader (BioTek Instruments, Inc., Burlington, VT).

\section{Alkaline Comet Assay}

The comet assay was performed according to the protocol established by the laboratory of Dr. Peter McKinnon (St. Jude Children's Research Hospital) [155]. In brief, 50,000 primary astrocyte or 30,000 human astroglial cells were seeded in Primaria 24-well plates (BD Bioscience, San Jose, CA) or in Falcon 24-well plates (BD Bioscience, San Jose, CA), respectively. Cells were allowed to attach overnight and then exposed to TG for three days. Thereafter, the cells were harvested and counted to prepare a $3 \times 10^{5}$ cells/ $\mathrm{ml}$ suspension in PBS. The cell suspension was mixed with $1.2 \%$ ultra-pure low melting point agarose (Invitrogen, Carlsbad, CA) and then casted onto chilled fully frosted glass slides (Fisher Scientific Co., Fair Lawn, NJ) pre-coated with $0.6 \%$ agarose (Bio-Rad, Richmond, CA). The cells were lysed for $1.5 \mathrm{hr}$ at $4{ }^{\circ} \mathrm{C}$ in lysis buffer (100 $\mu \mathrm{M}$ EDTA, $2.5 \mathrm{M} \mathrm{NaCl}, 10 \mathrm{mM}$ Tris, 1.3\% Triton X-100 and 3.3\% DMSO). The slides were washed twice with distilled $\mathrm{H}_{2} \mathrm{O}$ and incubated for $45 \mathrm{~min}$ at $4{ }^{\circ} \mathrm{C}$ in electrophoresis buffer (1 mM EDTA, $50 \mathrm{mM} \mathrm{NaOH}$ and 1\% DMSO). Electrophoresis was conducted at $12 \mathrm{~V}$ and $\sim 90 \mathrm{~mA}$ at $4^{\circ} \mathrm{C}$ for $25 \mathrm{~min}$. Slides were incubated for one hr in $400 \mathrm{mM}$ Tri-HCl neutralization buffer followed by a 20 min incubation in SYBR green I (Sigma-Aldrich, St. Louis, MO). Images were captured using a Nikon Eclipse TE300 fluorescent microscope. All experiments were replicated using two different sets of pooled cultures. The extent of DNA damage was expressed as the comet tail moment (the amount and distribution of DNA in the tail). The comet tail moment was measured in a minimum of 60 cells using CometScore version 1.5 (TriTek Corp., Sumerduck, VA).

\section{Immunohistochemistry}

Primary astrocytes were grown on poly-D-lysine chamber slides (BD Bioscience, San Jose, CA) for GFAP staining (to confirm astrocyte purity) or on glass coverslips and treated with 0 or $10 \mathrm{uM}$ of TG for $\gamma \mathrm{H} 2 \mathrm{AX}$ staining. Cells were fixed with $4 \% \mathrm{PFA} / \mathrm{PBS}$ for $10 \mathrm{~min}$, permeabilized with $0.5 \%$ Triton X-100/PBS for $5 \mathrm{~min}$. Cells were then washed with PBS and blocked with 3\% BSA/PBS for one hr. Cells were then immunostained with either anti-GFAP in PBS (1:500; Sigma-Aldrich, St. Louis, MO) for two hr or anti- $\gamma \mathrm{H} 2 \mathrm{AX}$ in 3\%BSA (1:1000, Millipore Co., Bedford, MA) for one hr. Subsequently, cells were washed with PBS and incubated for one hr with Cy2-conjugated donkey anti-mouse secondary antibody (1:250 in PBS containing 2\% donkey serum and 0.1\% Triton X-100; Jackson Immuno Research Laboratories, West Grove, PA) for GFAP or with donkey anti-mouse secondary antibody labeled with 1:800 Alexa 488 in 3\% BSA (Invitrogen, Carlsbad, CA) for one hr. Finally, vectashield mounting media (Vector Laboratories, Burlingame, CA) containing propidium iodide was used to counterstain nuclei. Slides were visualized using Nikon Eclipse TE300 fluorescent microscope. Slides stained for $\gamma \mathrm{H} 2 \mathrm{AX}$ were analyzed for $\gamma \mathrm{H} 2 \mathrm{AX}$ foci and a minimum of 100 cells were 
analyzed per genotype. Cells containing fewer than five foci were excluded to normalize for baseline staining observed in control treated cells. Cells with "pan-staining" were considered to have more than 5 foci per cell. Hence, the percentage of cells with $\gamma \mathrm{H} 2 \mathrm{AX}$ foci staining represents the percent of cells with greater than five foci per cell.

\section{Statistical Analysis}

Differences in cytotoxicity and DNA damage among all three groups were compared using Kruskal-Wallis analysis of ranks (Analysis of variance). Mann-Whitney $U$ test was used when only two groups were compared. Significance was declared when $p$ $<0.05$. All statistical analyses were performed using Statistica 8 (StatSoft Inc., Tusla, $\mathrm{OK})$.

\section{Results}

\section{Characterization of TPMT $^{+/+}$, TPMT $^{+/-}$, and TPMT ${ }^{-/-}$Primary Mouse Astrocyte Cultures}

The primary mechanism of thiopurine cytoxicity is believed to be through incorportation of TG nucleotide metabolites into DNA [39,152,156] (Figure 3-1), and therefore is dependent on the rate of cell proliferation. Hence, we first compared the doubling rates between TPMT $^{+/+}$, TPMT $^{+/}$, and TPMT $^{-/}$primary astrocyte cultures. Primary cultures were established from mouse cortices of each TPMT genotype and astrocyte purity was confirmed by GFAP (glial fibrillary acid protein, an astrocyte marker) fluorescent staining (Figure 3-3A) [157]. We found that there was no significant difference in growth rates of the different TPMT genotypes (Figure 3-3B). The mean doubling rates for $\mathrm{TPMT}^{+/+}, \mathrm{TPMT}^{+/-}$and $\mathrm{TPMT}^{-/-}$cultures were 33.9, 34.2, and 29.6 $\mathrm{hr}$, respectively $(\mathrm{p}=0.89)$. Next, we measured the level of TPMT protein activity using a well-established radiochemical assay that measures the conversion of 6-mercaptopurine to 6-methylmercaptopurine using [14C] S-adenosyl-L-methionine (SAM) as a methyl donor [105]. In agreement with previous studies comparing protein activity in hematological cells of different TPMT genotypes [121,150,158], protein activities in astrocytes were significantly different according to TPMT genotype (Figure 3-3C). $\mathrm{TPMT}^{+/+}, \mathrm{TPMT}^{+/-}$, and $\mathrm{TPMT}^{-/-}$primary astrocytes displayed high $\left(7.6 \mathrm{unit} / 10^{9} \mu \mathrm{g}\right.$ protein), intermediate (3.9 unit $/ 10^{9} \mu \mathrm{g}$ protein), and low $\left(0.25 \mathrm{unit} / 10^{9} \mu \mathrm{g}\right.$ protein) levels of protein activity, respectively $(\mathrm{p}=0.018)$. Based on these data, we next determined whether TPMT could modulate the cytotoxic effects of TG in primary astrocytes. 
A

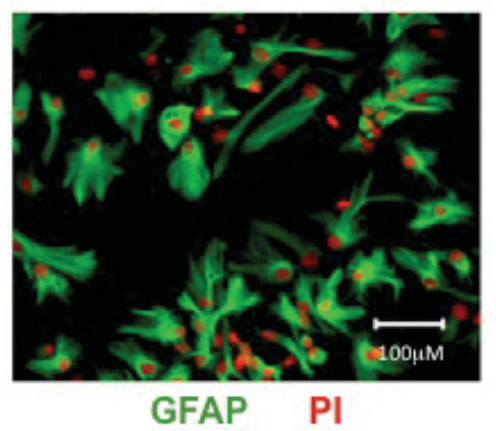

C

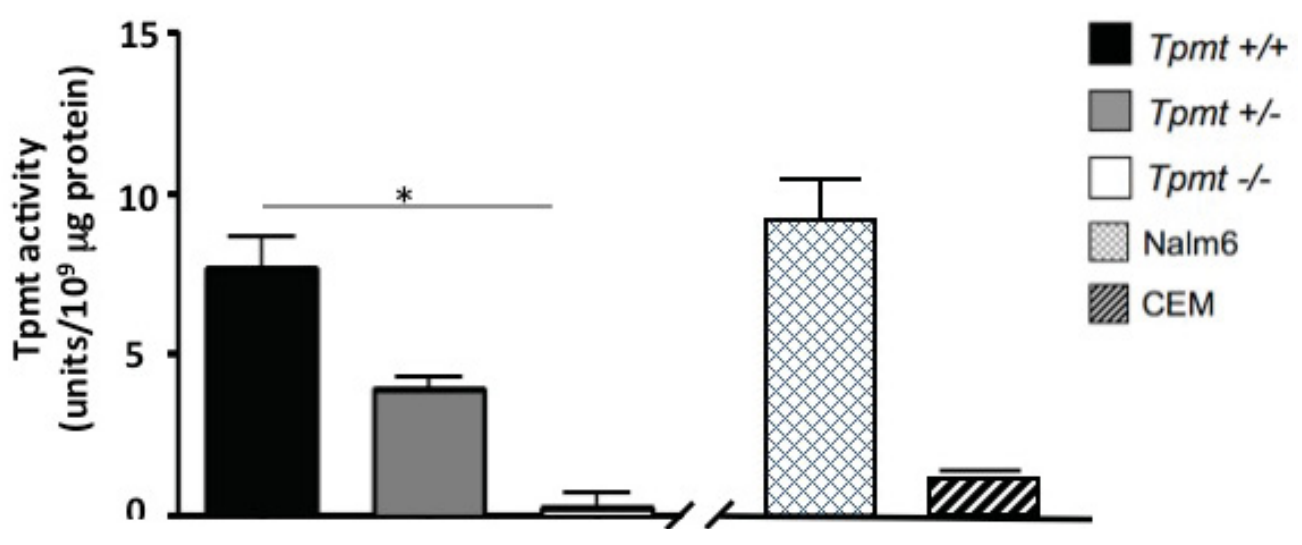

Figure 3-3. Characterization of TPMT $^{+/}$, TPMT $^{+/}$, and TPMT $^{-/-}$primary astrocyte cultures.

A, The image (200x) of primary astrocytes showing high astrocyte purity determined by GFAP (green) and propidium iodide (red) fluorescent staining. B, There was no significant difference in growth rates between astrocyte cultures of each TPMT genotype $(\mathrm{p}=0.89)$. $\mathbf{C}$, The level of TPMT protein activity was significantly different between the three TPMT genotypes $(p=0.018)$; and levels were comparable to Nalm6 and CEM control lymphoblastoid cell lines. Bars represent the means; whiskers represent the standard deviation $( \pm) ; * p<0.05$. Adapted with permission from Hosni-Ahmed A, Barnes JD, Wan J, Jones TS (2011) Thiopurine methyltransferase predicts the extent of cytotoxicty and DNA damage in astroglial cells after thioguanine exposure. PLoS ONE 6: e29163. 


\section{Comparison of TG-Associated Cytotoxicity in TPMT ${ }^{+/+}$, TPMT $^{+/}$, and TPMT $^{-/}$Primary Astrocyte Cultures}

To determine whether TPMT could predict thiopurine cytotoxicity in primary astrocytes, the MTT colorimetric assay was employed to compare TG sensitivity between astrocytes of each TPMT genotype. This assay measures cell viability by quantitating the extent at which metabolically active cells can reduce tetrazolium salt (MTT) to form purple formazan crystals. Astrocytes were exposed to escalating concentrations of TG $(0.125,1.25,12.5,25,50$, and $100 \mu \mathrm{M})$ for five days and then subjected to the MTT assay. Cell viability data at each $\mathrm{TG}$ concentration was used to calculate $\mathrm{IC}_{50}$ values. As expected, TG treatment resulted in cytotoxicity in a dose dependant manner (Figure 3-4A). Cell viability was significantly lower in $\mathrm{TPMT}^{+/-}$and $\mathrm{TPMT}^{-/-}$cultures at four concentrations of TG $(12.5,25,50$, and $100 \mu \mathrm{M})$ when compared to TPMT $^{+/+}$cultures (p $<0.04)$. Interestingly, $\mathrm{TPMT}^{+/}$but not $\mathrm{TPMT}^{-/}$astrocytes were significantly more sensitive at $1.25 \mu \mathrm{M}$ of TG than TPMT ${ }^{+/+}$astrocytes $(\mathrm{p}=0.004)$; and this finding was reflected in corresponding $\mathrm{IC}_{50}$ values. There was a 2.9 fold reduction in $\mathrm{IC}_{50}$ in $\mathrm{TPMT}^{+/-}$ $\left(3.5 \mu \mathrm{M}\right.$ TG) versus a 1.2 fold reduction in $\mathrm{TPMT}^{-/}(8.6 \mu \mathrm{M} \mathrm{TG})$ when compared TPMT ${ }^{+/+}$astrocytes $(10.3 \mu \mathrm{M} \mathrm{TG})$; and the $\mathrm{TPMT}^{+/-}$and $\mathrm{TPMT}^{-/-} \mathrm{IC}_{50}$ values were significantly different from TPMT ${ }^{+/+}(\mathrm{p}<0.05)$ (Figure 3-4B). Studies have shown that genotoxicity (i.e. DNA strand breaks) correlates with cytotoxicity after TG exposure $[116,156]$. Hence, we investigated whether the degree of TG-induced DNA strand breaks was associated with the level of cytotoxicity in primary astrocytes.

\section{Comparison of the Extent of DNA Damage Between TPMT $^{+/+}$, TPMT $^{+/}$, and TPMT $^{-/-}$Primary Astrocytes}

To address the question of whether the level of TG-induced cytotoxicity is correlated with DNA damage, we employed the alkaline comet assay. This assay quantifies DNA damage [i.e. single strand breaks (SSBs), double strand breaks (DSBs) and alkali labile sites] using fluorescent microscopy to visualize the extent of DNA migration from the nucleus of individual cells embedded in agarose [159]. We exposed astrocytes to $5 \mu \mathrm{M}$ of TG for $72 \mathrm{hr}$. $\mathrm{IC}_{50}$ data was used to select a dose that would allow us to capture damage in each genotype. We observed a significant difference in DNA damage between the three genotypes as measured by mean comet tail moment. Both TPMT $^{+/}$and TPMT $^{-/}$astrocytes had a significantly greater degree of DNA damage than $\mathrm{TPMT}^{+/+}$astrocytes (mean comet tail moment $=2.64,1.26$, and 0.4 , respectively; $\mathrm{p}=$ 0.033) (Figure 3-5A). In agreement with MTT studies, TPMT $^{+/-}$astrocytes were more sensitive to TG, as evidenced by a greater degree of DNA damage, than were TPMT $^{-/}$ astrocytes. The difference in DNA damage between TPMT $^{+/}$and TPMT $^{-/-}$astrocytes was not significantly different $(\mathrm{p}=0.083)$. There was no difference in DNA damage between TPMT genotypes of control treated astrocytes.

Studies have shown that the predominant DNA lesions caused by TG are SSBs although some DSB lesions are formed [156]. Therefore, we then compared $\gamma \mathrm{H} 2 \mathrm{AX}$ (an established DSB marker) foci staining after TG exposure to determine the extent of the 

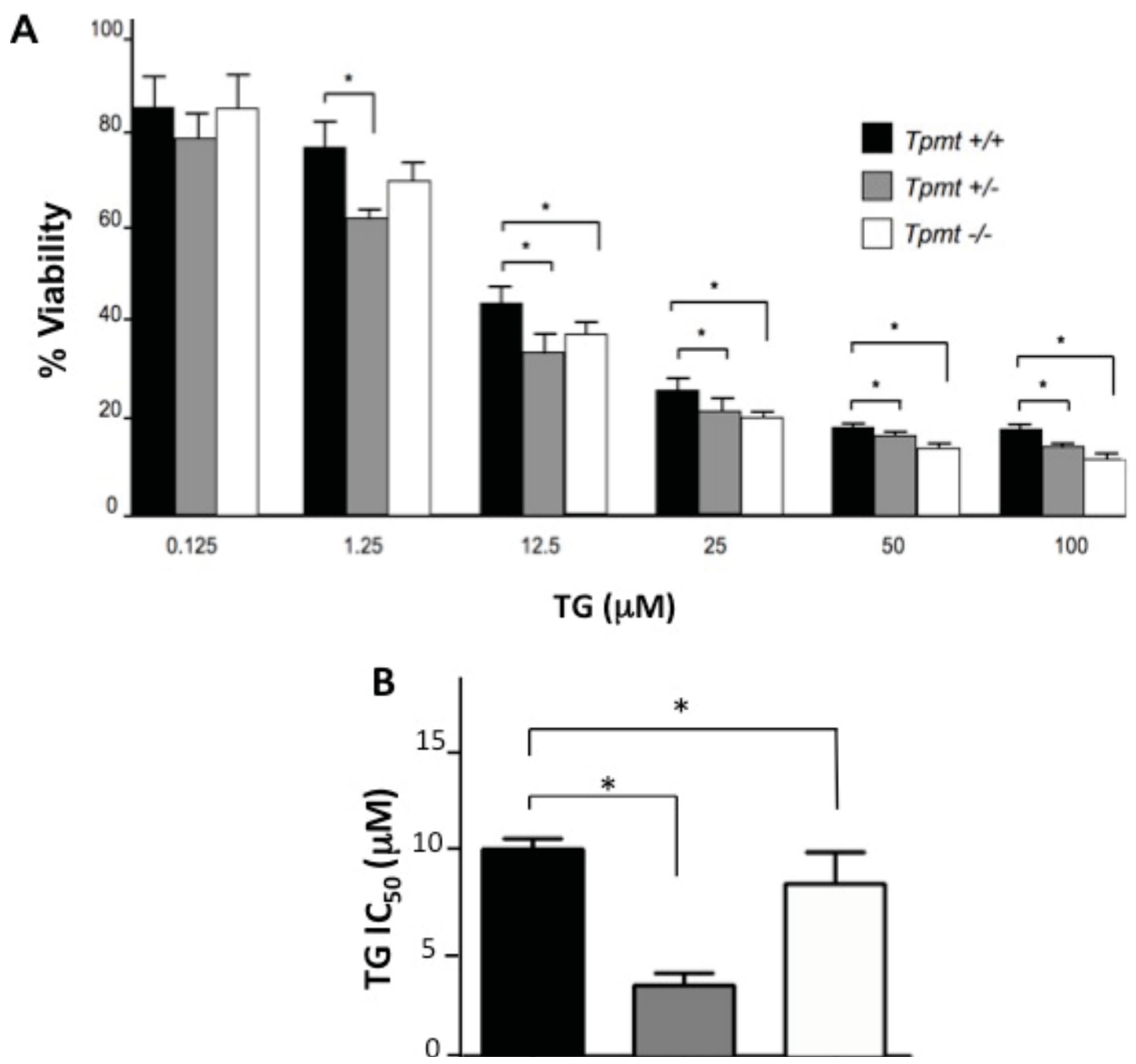

Figure 3-4. Comparison of TG-induced cytotoxicity in primary astrocyte cultures of each TPMT genotype.

A, Primary astrocytes of each TPMT genotype were exposed to thioguanine (TG) and cell viability was determined using the MTT assay. TPMT $^{+/}$and TPMT $^{-/-}$astrocytes were significantly more sensitive to TG $(12.5-100 \mu \mathrm{M})$ than $\mathrm{TPMT}^{+/+}(\mathrm{p}<0.04)$. B, $\mathrm{IC}_{50}$ values were significantly different between the three genotypes $(\mathrm{p}=0.027)$ and TPMT $^{+/}$ astrocytes were the most sensitive. Bars represent the means; whiskers represent the standard deviation $( \pm) ; * \mathrm{p}<0.05$. Adapted with permission from Hosni-Ahmed A, Barnes JD, Wan J, Jones TS (2011) Thiopurine methyltransferase predicts the extent of cytotoxicty and DNA damage in astroglial cells after thioguanine exposure. PLoS ONE 6: e29163. 

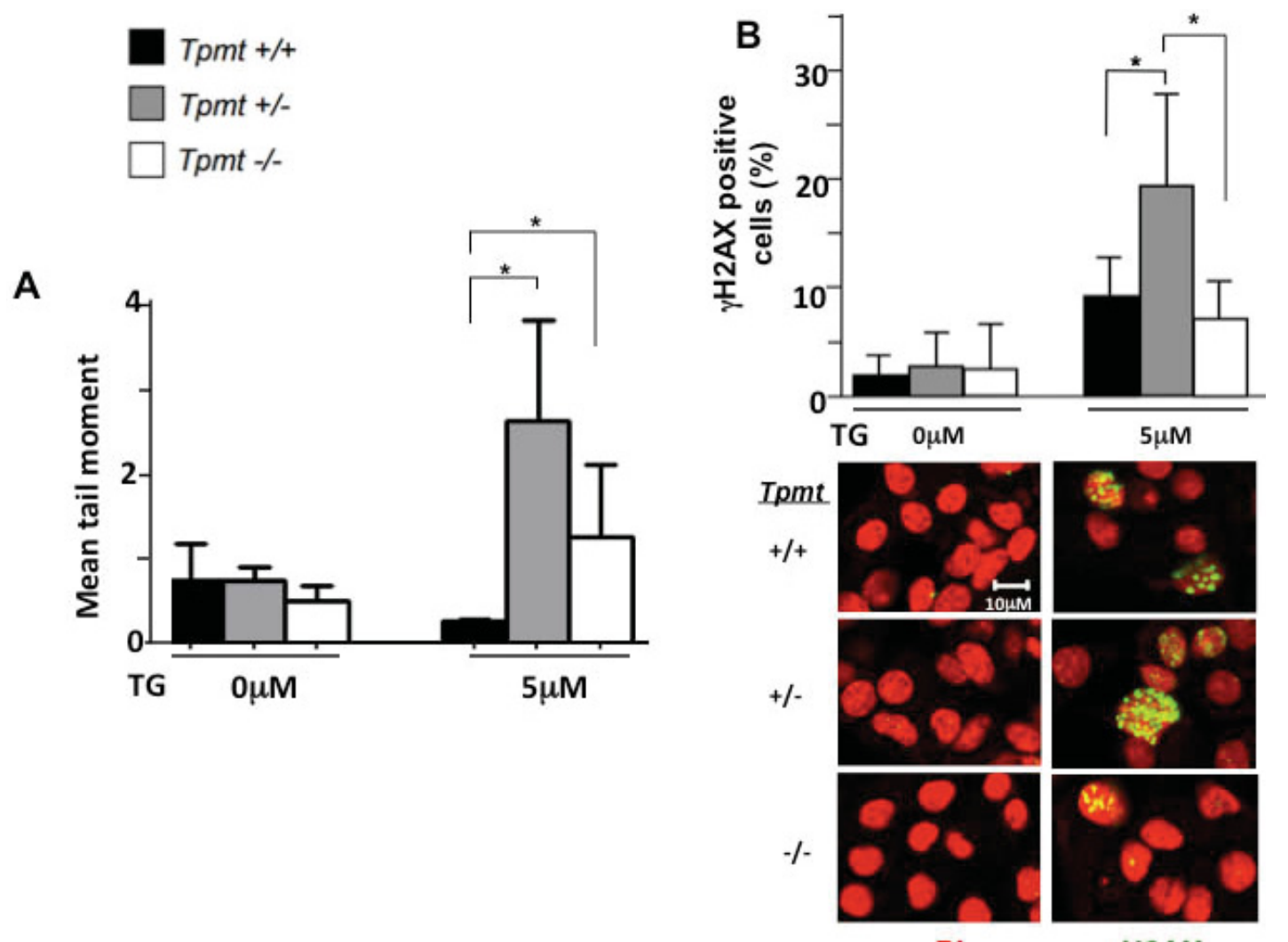

PI

$\gamma \mathrm{H} 2 \mathrm{AX}$

Figure 3-5. Comparison of TG-induced genotoxicity in primary astrocyte cultures of each TPMT genotype.

A, The alkaline comet assay was used to compare TG-induced DNA damage between TPMT genotypes. TPMT ${ }^{+/}$and TPMT $^{-/}$astrocytes had significantly higher mean comet tail moments than TPMT ${ }^{+/+}$astrocytes $(\mathrm{p}=0.033)$. B, Immunoflourescence staining for $\gamma \mathrm{H} 2 \mathrm{AX}$ confirms $\mathrm{IC}_{50}$ and comet data. TPMT ${ }^{+/}$astrocytes had greater damage than $\mathrm{TPMT}^{+/+}(\mathrm{p}=0.03)$. Below are representative images of $\gamma \mathrm{H} 2 \mathrm{AX}$ staining for control treated (left) and TG treated (right) astrocytes. $\gamma \mathrm{H} 2 \mathrm{AX}$ foci stained in green; nuclei stained in red (Zeiss LSM 710 Laser Scanning Microscope, 400x magnification). Bars represent the means; whiskers represent the standard deviation $( \pm) ; * p<0.05$. Adapted with permission from Hosni-Ahmed A, Barnes JD, Wan J, Jones TS (2011) Thiopurine methyltransferase predicts the extent of cytotoxicty and DNA damage in astroglial cells after thioguanine exposure. PLoS ONE 6: e29163. 
DNA lesions that are in the form of DSBs and whether this damage is different between the TPMT genotypes. Astrocytes were seeded on glass coverslips and exposed to either 0 or $5 \mu \mathrm{M}$ of TG. We found that TPMT ${ }^{+/}$astrocytes had the greatest degree of $\gamma \mathrm{H} 2 \mathrm{AX}$ foci staining (19.4\%) compared to TPMT ${ }^{+/+}(9.3 \%)$, and TPMT $^{-/-}(7.22 \%)$ (Figure 3-5B). In agreement with comet data, $\mathrm{TPMT}^{+/}$was the most sensitive genotype. There was a significant difference between TPMT ${ }^{+/}$and TPMT $^{+/+}(\mathrm{p}=0.03)$, but no significant difference was observed between TPMT $^{-/}$and TPMT $^{+/+}$cells.

\section{Comparison of TG Phenotypes Between Human Glioma Cell Lines with Different TPMT Protein Activities}

Next, we conducted conducted MTT and comet assays to assess TG-induced cytotoxicity and genotoxicity in human glioma cell lines with high and low TPMT activity and compared TG-induced cytotoxicity and DNA damage between them. First, we measured TPMT protein activity in four human glioma cell lines (T98, A172, U87, and LN18) to identify high and low TPMT expressers. We chose T98 (10.48 unit/10 $\mu \mathrm{g}$ protein) and A172 (6.15 unit/10 $\mu \mathrm{g}$ protein) as the high and low expressers, respectively (Figure 3-6A). We then subjected T98 and A172 to the MTT assay to measure TG-induced cytotoxicity. Both cell lines were exposed to increasing concentrations of TG $(0.15,0.75,1.5,6.25,12.5$ and $25 \mu \mathrm{M})$ for five days. The cell viability was determined for each concentration and these data were used to calculate $\mathrm{IC}_{50}$ values. As predicted by TPMT activity and in agreement with MTT studies performed in primary astrocytes, A172, the low TPMT expressing cell line, had significantly lower cell viabilities at each TG concentration as compared to T98 $(\mathrm{p}<0.005)$ (Figure 3-6B). The higher TG sensitivity of $\mathrm{A} 172$ was also reflected in the $\mathrm{IC}_{50}$ values. A172 displayed a 6.9 fold lower $\mathrm{IC}_{50}(1.2 \mu \mathrm{M})$ when compared to $\mathrm{T} 98(8.3 \mu \mathrm{M})$ (Figure 3-6C).

Next, we employed the comet assay to compare DNA damage between T98 and A172 cultures exposed to $3 \mu \mathrm{M}$ TG for $72 \mathrm{hr}$. In agreement with the primary astrocyte data, cells with low level of TPMT activity had significantly more DNA damage compared to high TPMT activity (mean comet tail moment: $\mathrm{A} 172=1$, T98 $=0.3 ; \mathrm{p}=$ 0.02) (Figure 3-6D). DNA damage in control treated cells was not significantly different between the two cell lines.

\section{Comparison of TG Phenotypes Between $\mathrm{A172}^{\text {mock }}$ and $\mathrm{A172}^{\mathrm{TPMT}}$ Isogenic Cell Lines}

To further validate our findings, TG-induced toxicity was compared using isogenic cell lines with different TPMT activities. A172 cells were the lowest TPMT expresser, hence we over-expressed TPMT protein in this cell line using lentiviral vector and compared TG-induced cytotoxicity and DNA damage between the mock transduced $\left(\mathrm{A} 172^{\text {mock }}\right.$ ) and TPMT transduced (A172 $2^{\text {TPMT }}$ ) cell lines. Transduction efficiency was monitored by GFP expression (Figure 3-7A). In addition, the level of TPMT protein activity in $\mathrm{A} 172^{\mathrm{TPMT}}$ was significantly higher as compared to $\mathrm{A} 172^{\text {mock }}$ (151.23 unit/109 $\mu \mathrm{g}$ protein versus $9.47 \mathrm{unit} / 10^{9} \mu \mathrm{g}$ protein, respectively; $\mathrm{p}<0.05$ ) (Figure 3-7B). We 

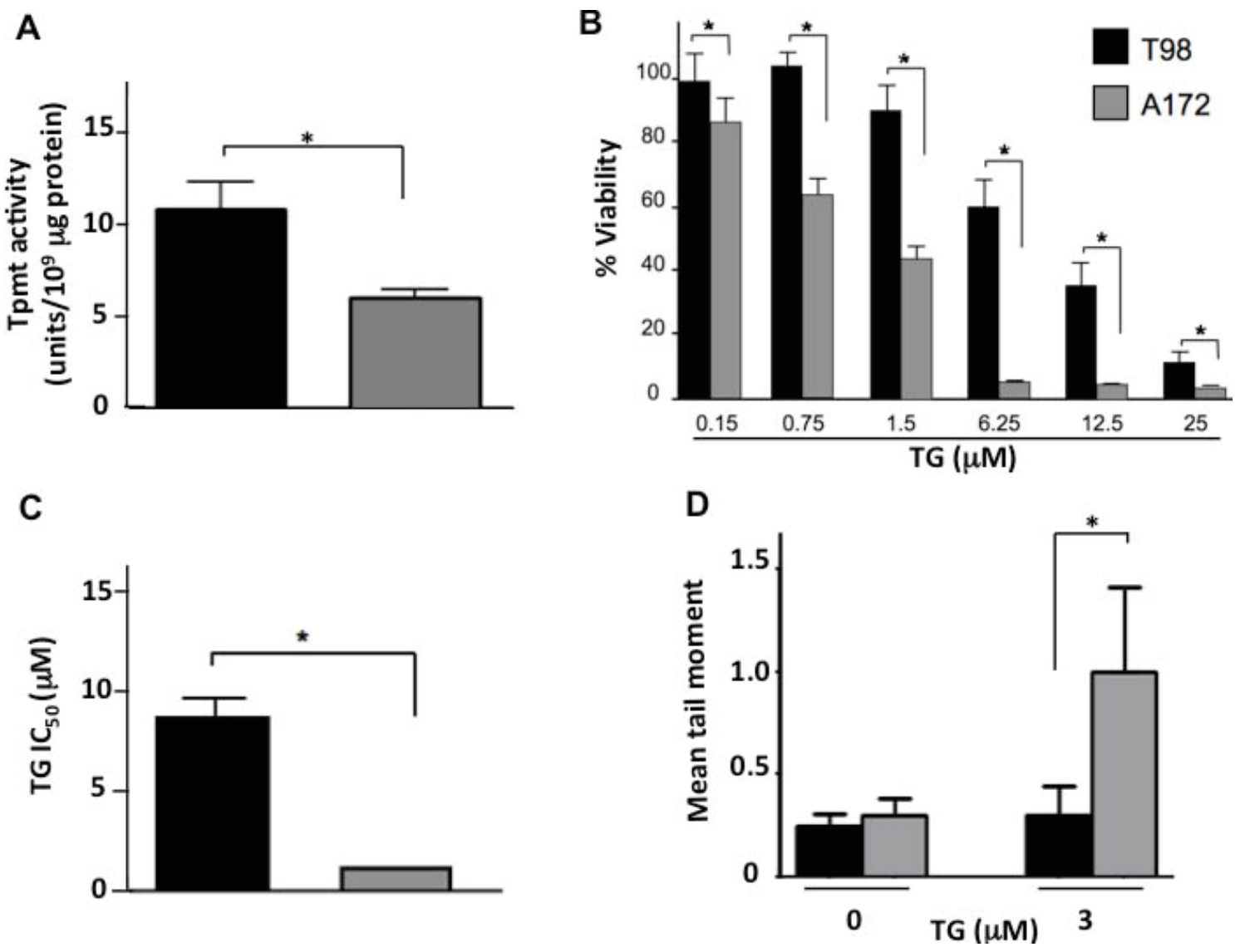

Figure 3-6. Assessment of TPMT-associated phenotypes in human glioma cell lines.

TPMT protein activity was measured in T98 and A172 glioma cell lines. A, T98 displayed a significantly higher level of protein activity than A172 $(p<0.05)$. B, As observed in primary astrocyte cultures, low TPMT protein activity is associated with greater cytotoxicity as determined by the MTT assay; $\mathbf{C}$, and reflected in $\mathrm{IC}_{50}$ values. A172 cells had a significantly more sensitive at all TG concentrations and lower $\mathrm{IC}_{50}$ than T98 ( $\mathrm{p}<0.05)$. D, The alkaline comet assay was used to compare TG-induced DNA damage. A172 displayed a significantly greater degree of TG-induced DNA strand breaks than T98 $(p=0.02)$ as measured by mean comet tail moment. Bars represent the means; whiskers represent the standard deviation $( \pm) ;{ }^{*} p<0.05$. Adapted with permission from Hosni-Ahmed A, Barnes JD, Wan J, Jones TS (2011) Thiopurine methyltransferase predicts the extent of cytotoxicty and DNA damage in astroglial cells after thioguanine exposure. PLoS ONE 6: e29163. 
Figure 3-7. TPMT-associated phenotypes in A172 isogenic cells.

TPMT was overexpressed in A172 cells using lentivial vector. A, Transduction efficiency was monitored by GFP expression. B, TPMT protein activity was measured in A172 ${ }^{\text {mock }}$ and $\mathrm{A} 172^{\mathrm{TPMT}}$ cell lines. TPMT protein activity was significantly higher in $\mathrm{A} 172^{\mathrm{TPMT}}$ ( $\mathrm{p}$ $<0.05)$. C, There were no significant differences in doubling times between A172 ${ }^{\text {TPMT }}$ and $\mathrm{A} 172^{\text {mock }}(\mathrm{p}=0.38)$. $\mathbf{D}, \mathrm{A} 172^{\mathrm{TPMT}}$ showed a significantly higher resistance to TG-induced toxicity when compared to $\mathrm{A} 172^{\text {mock }}$ as determined by the MTT cell viability data $(\mathrm{p}<0.005)$ and $\mathbf{E}$, by $\mathrm{IC}_{50}$ values $(\mathrm{p}<0.05)$. F , The alkaline comet assay was used to compare TG-induced DNA damage. A $172^{\text {mock }}$ displayed a significantly higher DNA damage than $\mathrm{A} 172^{\mathrm{TPMT}}(\mathrm{p}=0.03)$ as measured by mean comet tail moment. Bars represent the means; whiskers represent the standard deviation $( \pm) .{ }^{*} p<0.05$. Adapted with permission from Hosni-Ahmed A, Barnes JD, Wan J, Jones TS (2011) Thiopurine methyltransferase predicts the extent of cytotoxicty and DNA damage in astroglial cells after thioguanine exposure. PLoS ONE 6: e29163. 
A

\section{Bright field}

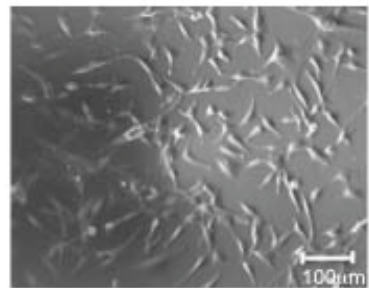

\section{GFP}

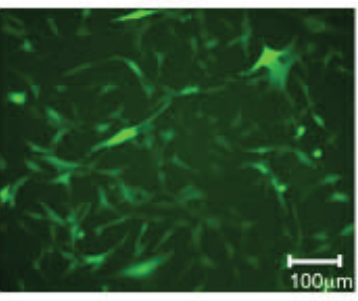

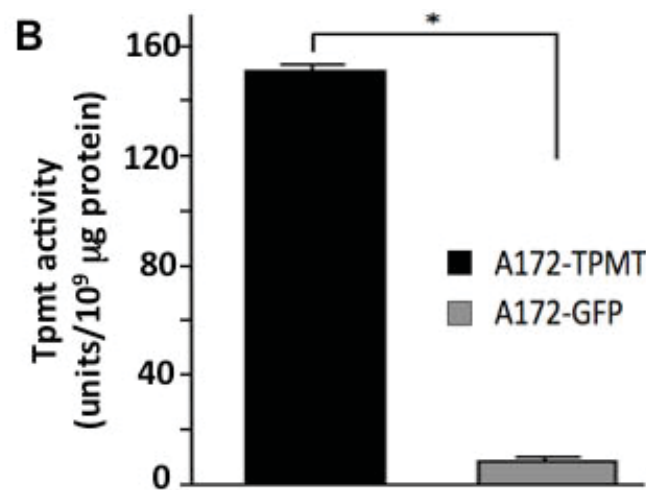

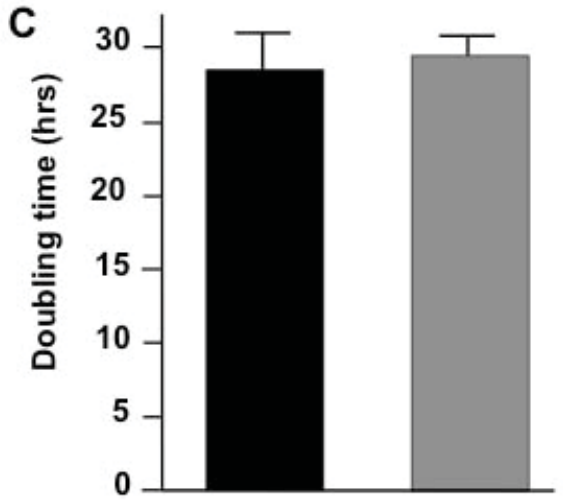




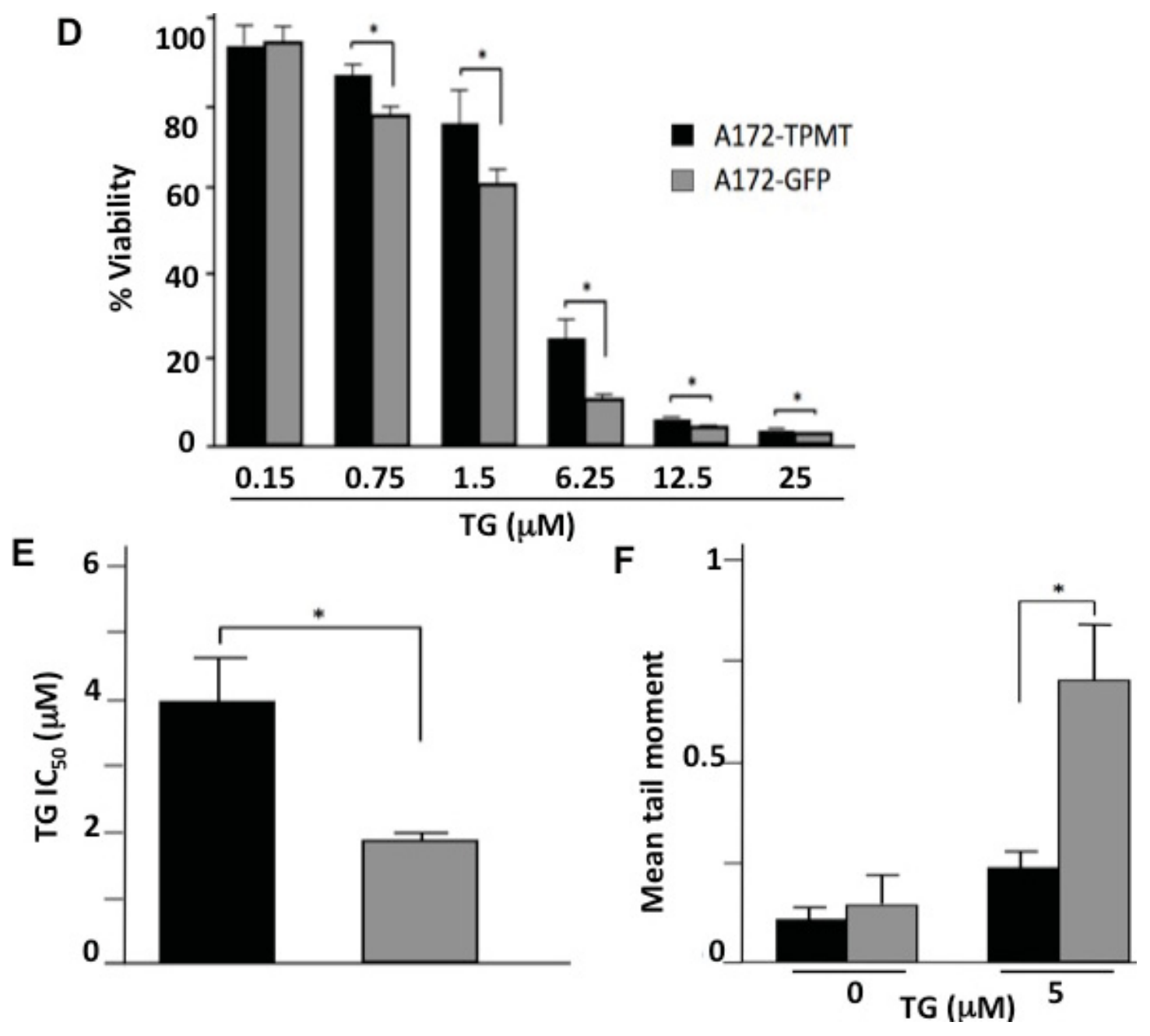

Figure 3-7. Continued. 
performed growth rate studies and found that there were no difference in doubling times between $\mathrm{A} 172^{\mathrm{TPMT}}$ and $\mathrm{A} 172^{\text {mock }}(28.5$ and $29.6 \mathrm{hr}$, respectively; $\mathrm{p}=0.38$ ) (Figure 3-7C). When $A 172^{\text {mock }}$ and $A 172^{\text {TPMT }}$ were exposed to increasing concentrations of TG we found that $\mathrm{A} 172^{\text {mock }}$ had significantly lower cell viabilities at $0.75,1.5,6.25,12.5$ and $25 \mu \mathrm{M}$ of TG as compared to $\mathrm{A} 172^{\mathrm{TPMT}}(\mathrm{p}<0.005)$ (Figure 3-7D). These findings were reflected in the $\mathrm{A} 172^{\mathrm{TPMT}} \mathrm{IC}_{50}$ value that was approximately two-fold higher than A172 ${ }^{\text {mock }}(3.9 \mu \mathrm{M}$ versus $1.9 \mu \mathrm{M}$, respectively; $\mathrm{p}<0.05)$ (Figure 3-7E).

Next, we exposed A172 $2^{\text {TPMT }}$ and $\mathrm{A} 172^{\text {mock }}$ to $5 \mu \mathrm{M}$ of TG for $72 \mathrm{hr}$ and compared the extent of DNA damage using the comet assay. We selected $5 \mu \mathrm{M}$ based on the IC50 value of $\mathrm{A} 172^{\mathrm{TPMT}}$. As expected, $\mathrm{A} 172^{\text {mock }}$ had a significantly greater degree of DNA damage when compared to A172 ${ }^{\mathrm{TPMT}}$ (mean comet tail moment: $\mathrm{A} 172^{\text {mock }}=0.7$, $\mathrm{A} 172^{\mathrm{TPMT}}=0.24 ; \mathrm{p}=0.03$ ) (Figure 3-7F). DNA damage in control treated cells was not significantly different between the two cell lines $(p=0.35)$. These data support our findings observed in both the primary mouse astrocytes and the glioma cell lines (T98 and wild-type A172) that when TPMT protein activity is low a greater degree of cytotoxicity and DNA damage can occur after TG exposure.

\section{Discussion}

TPMT is a cytoplasmic enzyme responsible for deactivating thiopurine drugs and a low TPMT phenotype can lead to life-threatening hematological toxicities when thiopurines are given. Indeed, chronic administration of thiopurines has been linked to an increased risk of cancer including brain tumors [47,48,50,52,67]. Unfortunately, not all patient populations that are treated with thiopurines are evaluated for their TPMT status prior to initiating thiopurine therapy; hence, the importance of TPMT to thiopurine-associated cancer risk is not clear. Since TPMT deficiency is relatively rare across populations, it is difficult to assess the importance of TPMT status to thiopurine therapy-related cancer risk. However, in a report by Relling et al. it was shown that whether patients developed a brain tumor as a late complication of post-antileukemic therapy depended on their TPMT status [47]. Patients who were determined to be at high risk for treatment relapse received prophylactic cranial irradiation concurrent with antimetabolite therapy; and those patients with a low TPMT had a surprisingly high incidence of brain tumors as a late complication. Indeed, ionizing radiation is a risk factor for brain tumorigenesis. However, in this report, patients with low TPMT had a $42.9 \%$ incidence of brain tumors compared to $15.8 \%$ for those with high TPMT.

Astrocytomas are the most common brain tumor type that has been reported in patients after thiopurine therapy $[47,48,50,67]$. To our knowledge, the importance of TPMT status to thiopurine-associated phenotypes in astroglial cells has not been investigated. We assessed TPMT and TG phenotypes in astroglial cells using primary mouse astrocytes from different TPMT genotypes and human glioma cells that differ in TPMT activity. In this study we have shown that TG induces cytotoxicity in a dose-dependent manner in astroglial cells; and cells with a low TPMT phenotype $\left(\mathrm{TPMT}^{+/-}\right.$and $\mathrm{TPMT}^{-/-}$astrocytes and $\mathrm{A} 172^{\mathrm{mock}}$ ) were significantly more sensitive to $\mathrm{TG}$. 
We also showed that TG exposure induced more DNA damage in the form of SSBs in astroglial cells with low TPMT than in cells with high TPMT. DNA damage in the form of DSBs was significantly different between astroglial cells with low and high TPMT. SSBs have been shown to be the primary DNA lesion induced by thiopurine drugs [156].

Interestingly, we found that low but not the complete absence of TPMT sensitized primary astrocytes to a higher degree of cytotoxicity and genotoxicity (i.e. $\mathrm{IC}_{50}$, $\mathrm{SSBs}$ and DSBs) after TG exposure. This discordance could possibly be explained by the induction of a compensatory mechanism that deactivates thiopurines upon the complete loss of TPMT. Another possible explanation could be a defect in the mismatch repair (MMR) system's ability to recognize DNA-TG nucleotide lesions. The MMR system is responsible for recognizing DNA mis-pairing caused by DNA-TG nucleotides and the subsequent signaling for cell death [160-163]. The latter possibility suggests that astroglial cells with a defective TPMT activity could have DNA lesions persisting in the genome that could potentially lead to mutagenesis and cancer. Interestingly, some studies suggest that children with TPMT heterozygosity develop brain tumors and other cancers more frequently than children with mutant TPMT. However, the frequency of heterozygous or mutant TPMT is very low making it is difficult to make any definitive conclusions $[47,164]$. It would be interesting to investigate whether TPMT status influences the extent of TG nucleotide incorporation into DNA and damage recognition by the MMR system as well as whether ionizing irradiation alone or in combination with thiopurines results in similar findings.

In summary, chronic thiopurine therapy has been associated with the development of brain cancer and TPMT status has been linked to brain tumor risk. We found that TPMT is an important factor for thiopurine drug-induced cytotoxicity and genotoxicity in astroglial cells. Because of the limited availability of human astrocytes we used human glioma cell lines. Although glioma cells can exhibit a number of genetic alternations that could contribute to the phenotypes observed, our study shows that TPMT is indeed an important determinant of thiopurine toxicity.

Low TPMT can increase the level of TG-induced genotoxic damage in both normal and in neoplastic astroglial cells. The observation that glioma cells can have variable degrees of TPMT protein activity and that low protein activity can sensitize cells to TG may suggest that thiopurines may potentially have a therapeutic role in low TPMT expressing glial tumors. However, since thiopurine drugs are linked with secondary brain tumors, we do not recommend the use of these agents in brain tumor treatment. Furthermore, In vitro studies have shown that thiopurine drugs are associated with mutagenic events in a variety of cell lines [116,151]. It is possible that the DNA damage caused by low TPMT function can ultimately contribute to transformative events over time. One study found that TPMT was not an important factor for cancer risk after thiopurine therapy [164]; however in this study only TPMT genotype was determined which may have excluded patients with discordance between TPMT genotype and activity. Individuals who are wild-type for TPMT can display a wide variation in their TPMT activity [127]. Further studies are needed to understand the importance of TPMT in the brain when thiopurines are given. Whether these findings can be replicated in vivo 
and whether factors important to DNA repair mechanisms are responsible for the TG phenotypes described in this study should also be investigated. 


\title{
CHAPTER 4. SCREENING OF NOVEL ANTI-GLIOMA SMALL MOLECULES*
}

\author{
Introduction
}

\section{Significance of Glioma Disease}

According to CBTRUS report, gliomas represent $80 \%$ of the malignant brain tumor incidence in the United States [2]. Furthermore, the overall survival rate is 3 years for less than $8 \%$ for grade IV GBM. On the global scale, the incidence of malignant brain tumors is approximately 3 in 100,000 per year, with a higher rate in the developed countries compared to the developing countries [4,5]. GBM is the most aggressive subtype of brain tumor and is characterized by diffuse infiltration into normal brain parenchyma making its resection very challenging. Consequently, these tumors can repopulate after surgery, causing cancer relapse [3]. Typical treatment involves surgical resection followed by cranial irradiation, which can alleviate the neurological symptoms and seizures in glioma patients. In general, patients receive 54-60 Gy radiotherapy regimen in 30 fractions during 6 weeks of treatment [3]. Usually concurrent TMZ therapy of $75 \mathrm{mg} / \mathrm{m}^{2} /$ day is given during the irradiation therapy followed by 28 cycles of adjuvant TMZ therapy $\left(150 \mathrm{mg} / \mathrm{m}^{2} /\right.$ day for 5 days/ week). Despite this aggressive therapy, there has not been a significant improvement in either the prognosis or the survival of patients. Therefore, there is an urgent need to find new therapeutical approaches to improve patients' survival and outcome. The current study focused on assessing the potential anti-cancer activity of 2 classes of small molecules; tetrahydroisoquinoline (THIQ) and chromene derivatives. A series of in vitro studies were conducted as part of the screening process to find the most potent derivatives with least toxicity to normal stromal cells.

\section{Tetrahydroisoquinoline Derivatives}

The laboratory of Dr. Duane Miller at UTHSC focuses on the synthesis of novel compounds against a wide variety of diseases and disorders including gliomas. In 2006, Mohler et al. have discovered novel THIQ derivatives that reduced cell viability in a rat glioma cell model [165]. According to this study, compound 1 (EDL-155) was the most toxic analog in glioma as well as lung, breast and cervical cancer cell lines. The $\mathrm{IC}_{50}$ values ranged from approximately 3-10 $\mu \mathrm{M}$, depending on the cancer cell line. Therefore,

\footnotetext{
*Adapted with permission. Patil R, Hosni-Ahmed A, Jones T, Patil S, Asres L, et al. (2014) Synthesis and In Vitro Evaluation of Novel 1,2,3,4-Tetrahydroisoquinoline Derivatives as Potent Antiglioma Agents. Anticancer Agents Med Chem (In Press). Patil SA, Wang J, Li XS, Chen J, Jones TS, et al. (2012) New substituted 4H-chromenes as anticancer agents. Bioorg Med Chem Lett 22: 4458-4461.
} 
we were interested in investigating the anti-cancer activity of other THIQ analogs in a panel of human glioma cell lines [166].

\section{Chromene Derivatives}

One of the limiting factors of cancer therapeutics is poor drug penetration. Previous studies showed an improvement in drug penetration as well as efficacy when Arginine-Glycine-Aspartic Acid (RGD) peptides were co-injected with anti-cancer drugs [167]. Drs. Miller and Patil research groups used RGD and similar analogs to search online libraries for similar anti-tumor small molecules [168]. From the 2 hits obtained, GRI-394837, a chromene analog was subsequently investigated. Chromenes are analogs of podophyllotoxin, a natural plant product, that have been isolated from Podophyllum species [169]. Whereas, podophyllotoxin function in plants is not fully understood, its semisynthetic analogs exhibit anti-cancer activity. Chromenes demonstrated high efficiency as apoptosis inducers by inhibiting microtubule assembly and as vascular disrupting agents (VDA) [170,171]. In the current study, several chromene derivatives have been synthesized and their activities in glioma were assessed in vitro and in vivo.

\section{Materials and Methods}

\section{Drug Preparation}

THIQ and chromene small molecules have been synthesized in Dr. Miller and Dr. Patil's laboratories respectively at UTHSC. For in vitro screening, a freshly prepared 50 $\mu \mathrm{M}$ stock solution was prepared by dissolving EDL-360 in DMSO. EDL-291 was dissolved in PBS to prepare $10 \mu \mathrm{M}$ stock solution. Other THIQs, chromenes and TMZ compounds were dissolved in DMSO. A freshly prepared $100 \mu \mathrm{L}$ stock solution was prepared by dissolving the compound in DMSO. For treatment of tumor-bearing mice, EDL-360 was dissolved in 0.9\% saline, whereas SP-6-27 was dissolved in glacial acetic acid.

\section{Glioma Cell Lines and Primary Astrocyte Cultures}

The human glioma cell lines, T98, U87 and LN18, were obtained from the American Type Culture Collection (Manassas, VA, USA). A172 glioma culture was a generous gift from Dr. C. Duntsch (Department of Neurosurgery, UTHSC). T98 and U87 cultures were grown in Minimum Essential Medium Eagle (Cellgro, Hemdon, VA), supplemented with 10\% fetal bovine serum, $2 \mathrm{mM}$ L-glutamine, $0.1 \mathrm{mM}$ nonessential amino acids, $1 \mathrm{mM}$ sodium pyruvate, $100 \mathrm{IU} / \mathrm{ml}$ penicillin, and $100 \mu \mathrm{g} / \mathrm{ml}$ streptomycin. A172 cells were grown in Dulbecco's Modified Eagle's Medium (Cellgro, Hemdon, VA), supplemented with 10\% fetal bovine serum, $2 \mathrm{mM}$ L-glutamine, $100 \mathrm{IU} / \mathrm{ml}$ 
penicillin, and $100 \mu \mathrm{g} / \mathrm{ml}$ streptomycin. All cell cultures were maintained at $37^{\circ} \mathrm{C}$ in a $5 \%$ $\mathrm{CO}_{2}$ humidified atmosphere.

Astrocyte cultures were established from 2-5 day old mice. A dissecting microscope was used to remove the meninges and hippocampus, and the cortices were mechanically dissociated. Subsequently, cultures were established and maintained in Dulbecco's modified Eagle's medium/Ham's F-12 50/50 mix (Cellgro, Hemdon, VA) supplemented with $10 \%$ fetal bovine serum, $2 \mathrm{mM}$ L-glutamine, $100 \mathrm{IU} / \mathrm{ml}$ penicillin, $100 \mu \mathrm{g} / \mathrm{ml}$ streptomycin and $20 \mathrm{ng} / \mathrm{ml}$ epidermal growth factor (Millipore Co., Bedford, MA) and grown in Primaria flasks, (BD Bioscience, San Jose, CA). After three days of incubation, cultures were fed with $20 \mathrm{ng} / \mathrm{ml}$ epidermal growth factor and the media was changed after five days. At passage 2 three individual primary cultures of the same genotype were pooled and seeded for experiments. Only cultures with greater than $80 \%$ astrocyte purity as determined by GFAP immunohistochemistry were included in experiments.

\section{MTT Assay}

Cell viability was measured using 3-(4,5-dimethylthiazol-2-yl)-2,5-diphenyl tetrazolium bromide (MTT) (Sigma-Aldrich, St. Louis, MO). In brief, 1000 cells were seeded per well in 96-well plates. Plates were pre-coated with poly-L-ornithine (Sigma-Aldrich, St. Louis, MO) and laminin (Invitrogen, Carlsbad, CA) for primary astrocytes to facilitate cell attachment. Cells were allowed to attach overnight and small molecules or vehicle control was added to the appropriate wells. After five days, MTT was added and allowed to incubate for $3 \mathrm{hr}$. The media was carefully aspirated and the purple formazan crystals were dissolved in $100 \mu 1$ DMSO (Fisher Scientific Co., Fair Lawn, NJ). Absorbance was measured at 570/690 nm using a FLx800 fluorescence microplate reader (BioTek Instruments, Inc., Burlington, VT). A series of experiments were conducted to determine the concentration range for each compound to generate a half-maximal inhibitory concentration $\left(\mathrm{IC}_{50}\right)$.

\section{Lentiviral Transduction}

Luciferase cDNA was amplified by PCR, purified through agarose gel electrophoresis, and subcloned into BamHI and EcoRI sites of pLenti-pgk-puro vector (Viral vector core, UTHSC) (Figure 4-1A). The pLenti-Luc-mKate-pgk-puro and pLenti-pgk-puro lentiviral vectors were packaged in 293T cells [154]. The lentiviral vectors were used to transduce the U87 glioma cell line in the presence of $6 \mu \mathrm{g} / \mathrm{mL}$ polybrene (Sigma-Aldrich, St. Louis, MO) (Figure 4-1B). Stably transfected cells were then selected by growing the cells in $1 \mu \mathrm{g} / \mathrm{ml}$ puromycin. Transduction efficiency was monitored by using fluorescent microscope. 

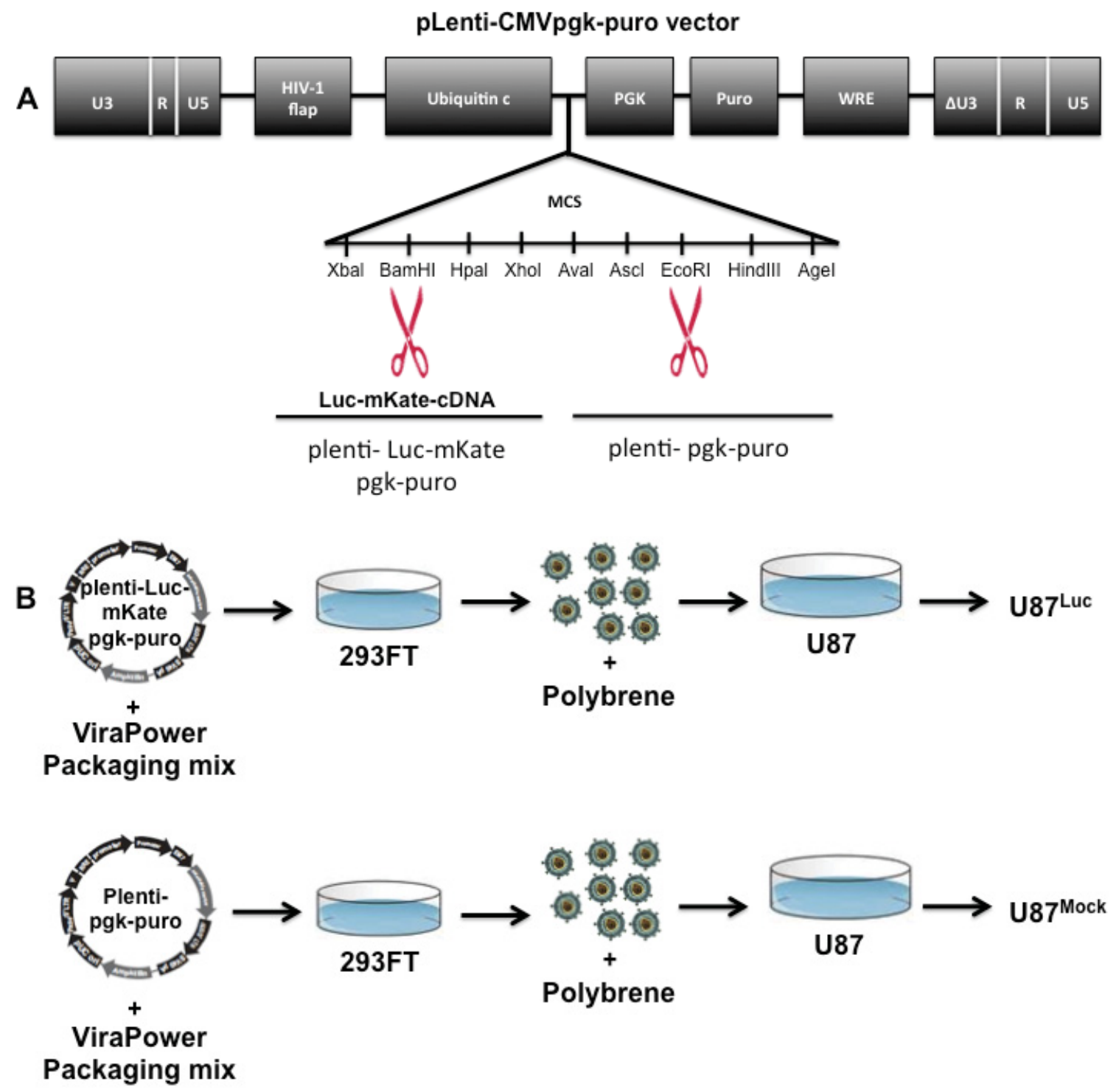

\section{Figure 4-1. pLenti-pgk viral vector.}

A, Luciferase and mkate cDNA were subcloned into pLenti-pgk-puro vector using BamHI and EcoRI sites. B, The lentivial vectors were packaged in $293 \mathrm{~T}$ cells. The mock- (U87 ${ }^{\text {mock }}$ ) and Luciferase-transduced (U87 ${ }^{\text {Luc }}$ ) cell lines were generated by transducing U87 cells with either pLenti-Luc-mkate-pgk-puro or pLenti-pgk-puro lentivial vector (empty vector), respectively. 


\section{Luciferase Activity}

Luciferase activity was measured in U87 transduced cells using dual-luciferase reporter assay (Promega, Madison, WI). U87 ${ }^{\text {Luc }}$ and U87 ${ }^{\text {mock }}$ were seeded in 6-well plates and allowed to reach $70 \%$ confluency. After removing cell medium and washing once with PBS, the cells were lysed in $500 \mu \mathrm{L} 1 \mathrm{X}$ passive lysis buffer (PLB) for $15 \mathrm{~min}$ at room temperature with gentle shaking. Subsequently, $10 \mu \mathrm{L}$ of PLB lysate was mixed with $50 \mu \mathrm{L}$ LARII (luciferase substrate) and firefly luciferase activity was determined using GloMax- Multi Jr Single-Tube Multimode Reader (Promega, Madison, WI).

\section{In Vivo Drug Testing}

A glioma xenograft mouse model was generated by injecting $\mathrm{U} 87^{\mathrm{Luc}}$ cells

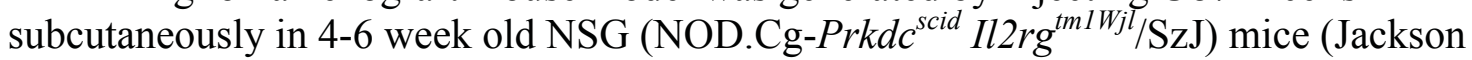
Laboratory, Bar Harbor, ME. The mice were anesthetized using isoflurane and subcutaneously injected with $100 \mu \mathrm{L}$ containing $10^{7}$ U87 ${ }^{\mathrm{Luc}}$ cells in the right flank of the mice. Once the tumors were palpable, the mice were divided randomly into groups of 5 mice (i.e. untreated, vehicle treated, and drug treated). Subsequently, $100 \mu \mathrm{L}$ of the test compounds were administered intratumorally. Tumor size, body weight and bioluminescence signal were obtained twice/week. In addition, non-invasive bioluminescent imaging was acquired biweekly using the IVIS-200 Imaging System (Xenogen Corporation, Berkeley, CA). The studies were terminated when the tumors reached $10 \%$ of total body weight.

\section{Statistical Analysis}

Mann-Whitney $U$ test was used when only two groups were compared. The results were considered significant when $\mathrm{p}<0.05$. All statistical analyses were performed using Statistica 8 (StatSoft Inc., Tusla, OK).

\section{Results}

\section{In Vitro Screening of THIQ Analogs}

The anti-cancer activity of THIQ analogs in human glioma cell lines was investigated in vitro using MTT cell viability assays. In order to determine whether the compounds had toxicity in normal brain cells, astrocyte cultures were generated from the cortex of normal mice. Both glioma and astrocytes cultures were plated and treated under the same conditions. A series of MTT assay were performed to find the dosing range for each compound to calculate the $\mathrm{IC}_{50}$ values.

TMZ is the standard chemotherapy for treating glioma and was used in the current study as a reference to evaluate the anti-cancer efficacy of our compounds. T98, U87, 
LN18 and A172 glioma cell lines were more sensitive to TMZ compared to normal astrocytes (Figure 4-2A). At a concentration $1000 \mu \mathrm{M}$, TMZ did not induce $50 \%$ death of normal astrocytes. T98 and LN18 cells were more resistant to TMZ compared to U87 and A172 cells. When glioma cell lines were treated with THIQ compounds, cell death was induced in a dose dependent manner. EDL-155 was very potent against glioma cell lines induced $\sim 95 \%$ cell death at $20 \mu \mathrm{M}$ (Figure 4-2B). However, it was quite toxic to normal astrocytes as well $\left(\mathrm{IC}_{50}=5.2 \mu \mathrm{M}\right)$. LN18 was the only cell line that EDL-155 markedly inhibited cell viability at all the concentrations tested and had a significantly lower $\mathrm{IC}_{50}$ compared to astrocytes $(\mathrm{p}<0.05)$. EDL-323 was significantly more toxic to LN18 and A172 cells than normal astrocytes $(\mathrm{p}<0.05)$ and completely inhibiting their viability at $80 \mu \mathrm{M}$ (Figure 4-2C). Similar to EDL-323, EDL-291 analog significantly reduced the viability of $\mathrm{LN} 18$ and $\mathrm{A} 172$ cells $\left(\mathrm{IC}_{50}=37\right.$ and $41.2 \mu \mathrm{M}$, respectively) compared to normal astrocytes $\left(\mathrm{IC}_{50}=51.1 \mu \mathrm{M}\right)(\mathrm{p}<0.05)$ (Figure 4-2D). EDL-355 showed better anti-cancer activity compared to EDL-291 and EDL-323 (Figure 4-2E). It had significantly lower $\mathrm{IC}_{50}$ value in $\mathrm{LN} 18$ cells $\left(\mathrm{IC}_{50}=7.3 \mu \mathrm{M}\right)$ compared to normal astrocytes $\left(\mathrm{IC}_{50}=10.3 \mu \mathrm{M}\right)$. However, EDL-358 was more selective toxicityto glioma cell lines (Figure 4-2F). It had a significantly higher $\mathrm{IC}_{50}$ in normal astrocytes compared to all glioma cell lines $(\mathrm{p}<0.05)$. EDL-360 showed a higher cytotoxic action in glioma cell lines $\left(\mathrm{IC}_{50}=5.4-10,4\right) \mu \mathrm{M}$ compared to EDL-358 $\left(\mathrm{IC}_{50}=8.25-24.6\right)$. In addition, it significantly induced cell death in all glioma cell lines compared to normal astrocytes $(\mathrm{p}$ $<0.05$ ) (Figure 4-2G). Further modification in EDL-361 resulted in an increased toxicity in normal astrocytes, and hence a decrease in selective toxicity for glioma cell lines (Figure 4-2H).

\section{In Vitro Screening of Chromene Analogs}

Next, we assessed the anti-glioma toxicity of chromene analogs in vitro. MTT cell viability assay showed high anti-glioma activity of SP-6-19. Greater than $90 \%$ of glioma cells were dead at a $10 \mu \mathrm{M}$ concentration, whereas the same dose killed $<50 \%$ of normal astrocyte cells (Figure 4-3A). The observed cytotoxicity in glioma cell lines was in nanomolar range $\left(\mathrm{IC}_{50}<80 \mathrm{nM}\right)$. In contrast, a concentration of $9.5 \mu \mathrm{M}$ was required to reach an $\mathrm{IC}_{50}$ in normal astrocytes. It appeared to be most toxic in A172 cell line compared to LN18 and U87 cells. SP-6-21 was less toxic compared to SP-6-19. However, the observed cytotoxicity remained in the submicromolar range in glioma cell lines (Figure 4-3B). All the test glioma cell lines appear to respond equally to the toxic action of this analog. SP-6-23 exhibited even less toxicity compared to SP-6-21. IC 50 values ranged from 0.7-0.9 $\mu \mathrm{M}$ in all glioma cell lines (Figure 4-3C). Approximately, $90 \%$ cell death was observed in T98 and U87 cells at $20 \mu \mathrm{M}$, whereas, in LN18 and A172 was $\sim 5 \%$ at the same concentration. Chromene analog, SP-6-25, exerts anti-glioma activity in micromolar range $(\sim 1.2 \mu \mathrm{M})$ (Figure 4-3D). Interestingly, astrocytic cell death was only observed at the $100 \mu \mathrm{M}$ concentration. Approximately, a 60-fold higher concentration is needed to reach $\mathrm{IC}_{50}$ in normal astrocytes. Interestingly, $\mathbf{S P - 6 - 2 7}$ was the most potent chromene analog (Figure 4-3E). There was an approximately $90 \%$ reduction of glioma cell viability at $0.1 \mu \mathrm{M}$, while in astrocytes the $\mathrm{IC}_{50}$ was $\sim 2 \mu \mathrm{M}$. All tested glioma cell lines had a significantly lower $\mathrm{IC}_{50}$ values compared to normal astrocytes $(\mathrm{P}<$ 
Figure 4-2. The effect of THIQ analogs and TMZ on the cell viability of gliomas and astrocytes.

A, The glioma cell lines were more sensitive to $\mathbf{T M Z}$ compared to normal astrocytes. $\mathbf{B}$, THI analog; EDL-155 was more toxic to both normal astrocytes and human glioma cell lines compared to TMZ. C, EDL-323 derivative showed less potency against glioma cell lines when compared to EDL-155. However, LN18 and A172 had a significant less viabilities and $\mathrm{IC}_{50}$ compared to normal astrocytes. D, EDL-291 analog exhibited a similar activity profile to EDL-323 in terms of toxicity and selectivity. $\mathbf{E}$, Methoxy meta-substitution (EDL-355) enhanced the toxicity, however the selectivity remained poor. F, Methoxy para-substitution (EDL-358) had similar toxicity pattern against glioma lines compared to EDL-155. More importantly, EDL-358 had a significant higher $\mathrm{IC}_{50}$ in normal astrocytes compared to for all glioma cell lines. $\mathbf{G}$, Adding one carbon atom to the carbon linker between biphenyl and THI ring (EDL-360; $n=1)$ improved both toxicity and selectivity of EDL-360 analog. H, Further increasing the length of the carbon linker (EDL-361; $\mathrm{n}=2$ ) reduced the toxicity against glioma cell lines when compared to EDL-360 and showed almost equal potency to normal astrocytes. MTT cell viability using escalated doses of the test compounds (left panels). Bar graphs comparing the $\mathrm{IC}_{50}$ data for normal mouse astrocytes and human glioma cell lines (right panels). Bars represent the means; whiskers represent the standard deviation $( \pm),{ }^{*} \mathrm{p}<0.05$. 


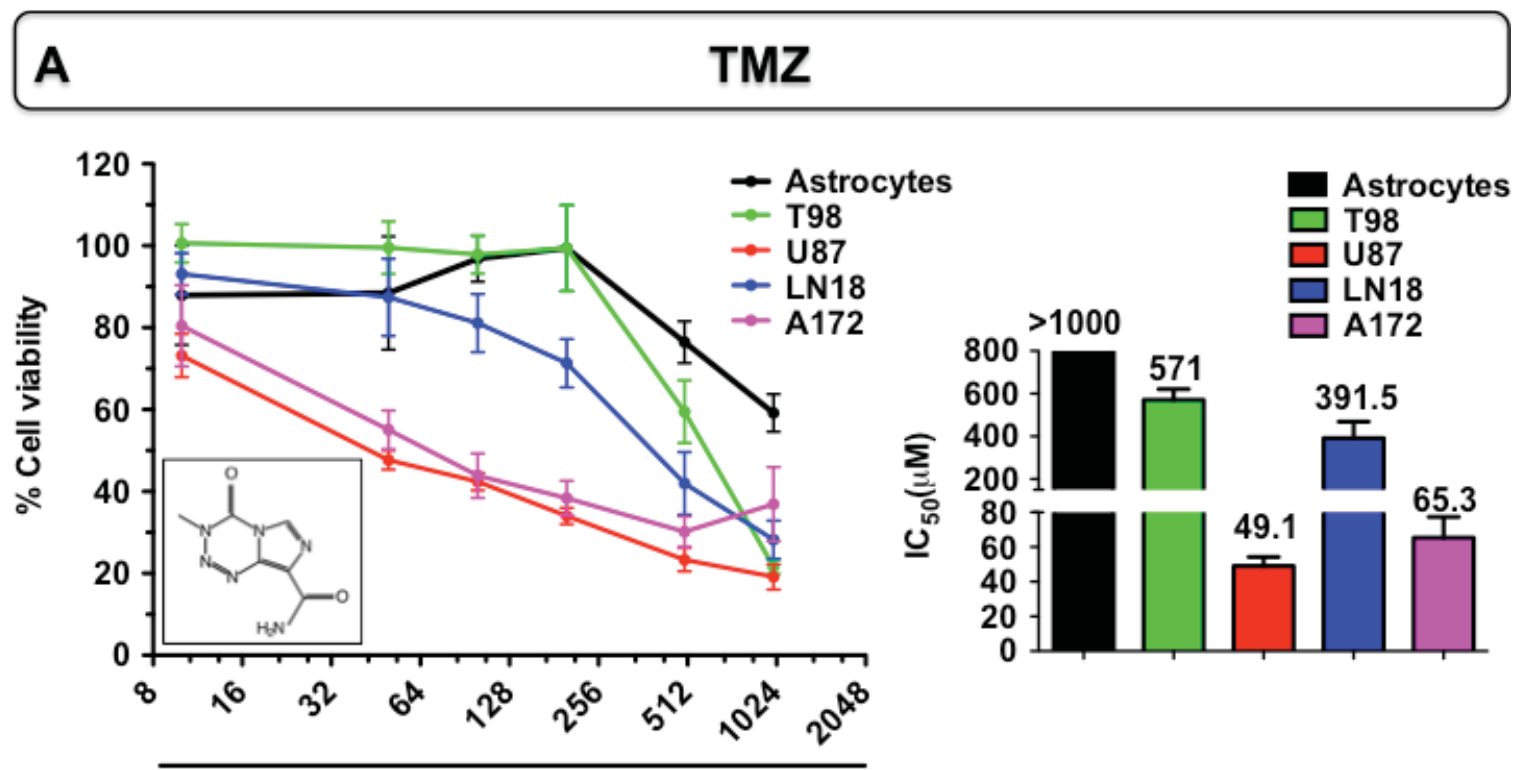

TMZ $(\mu M)$

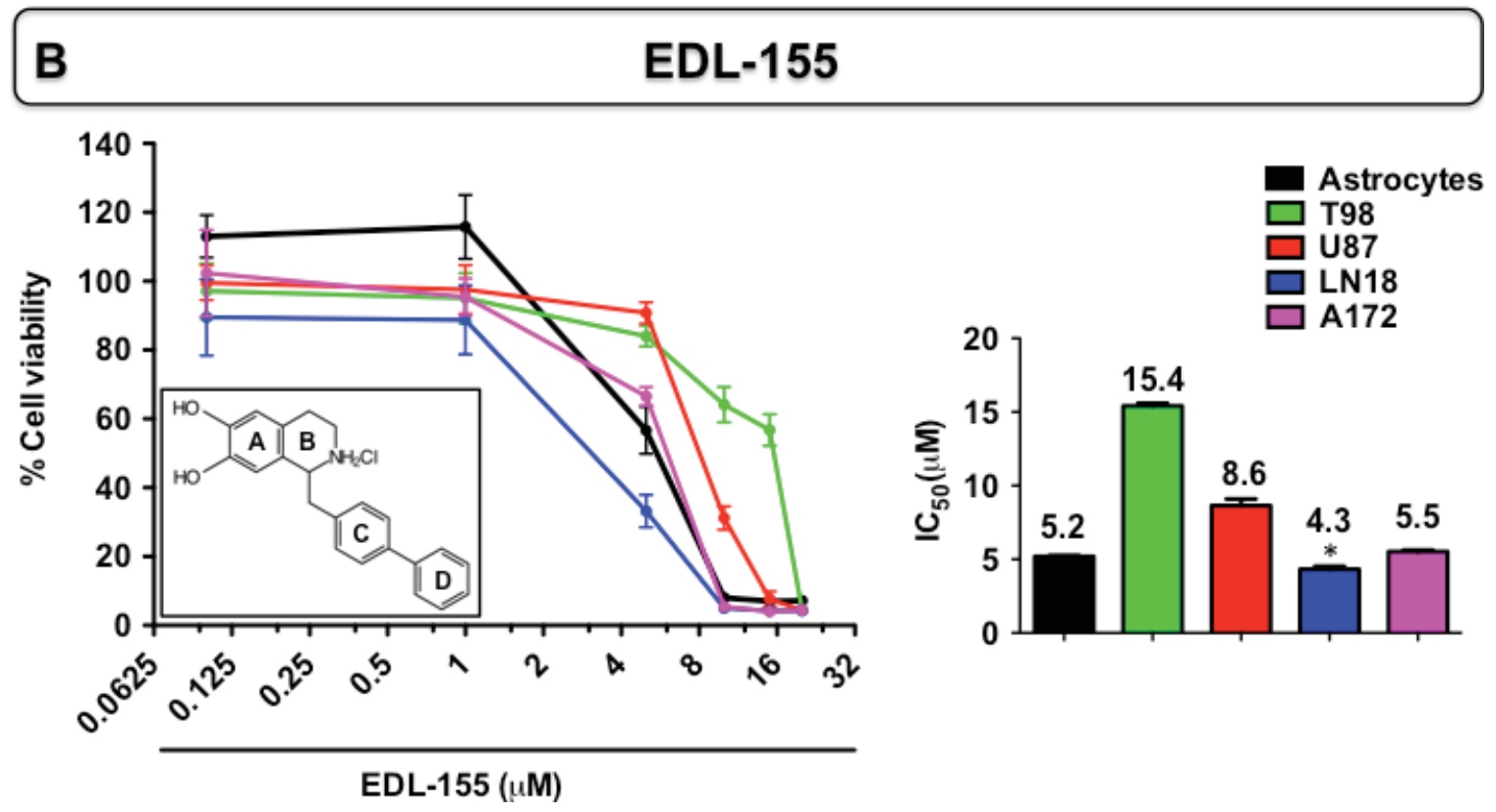



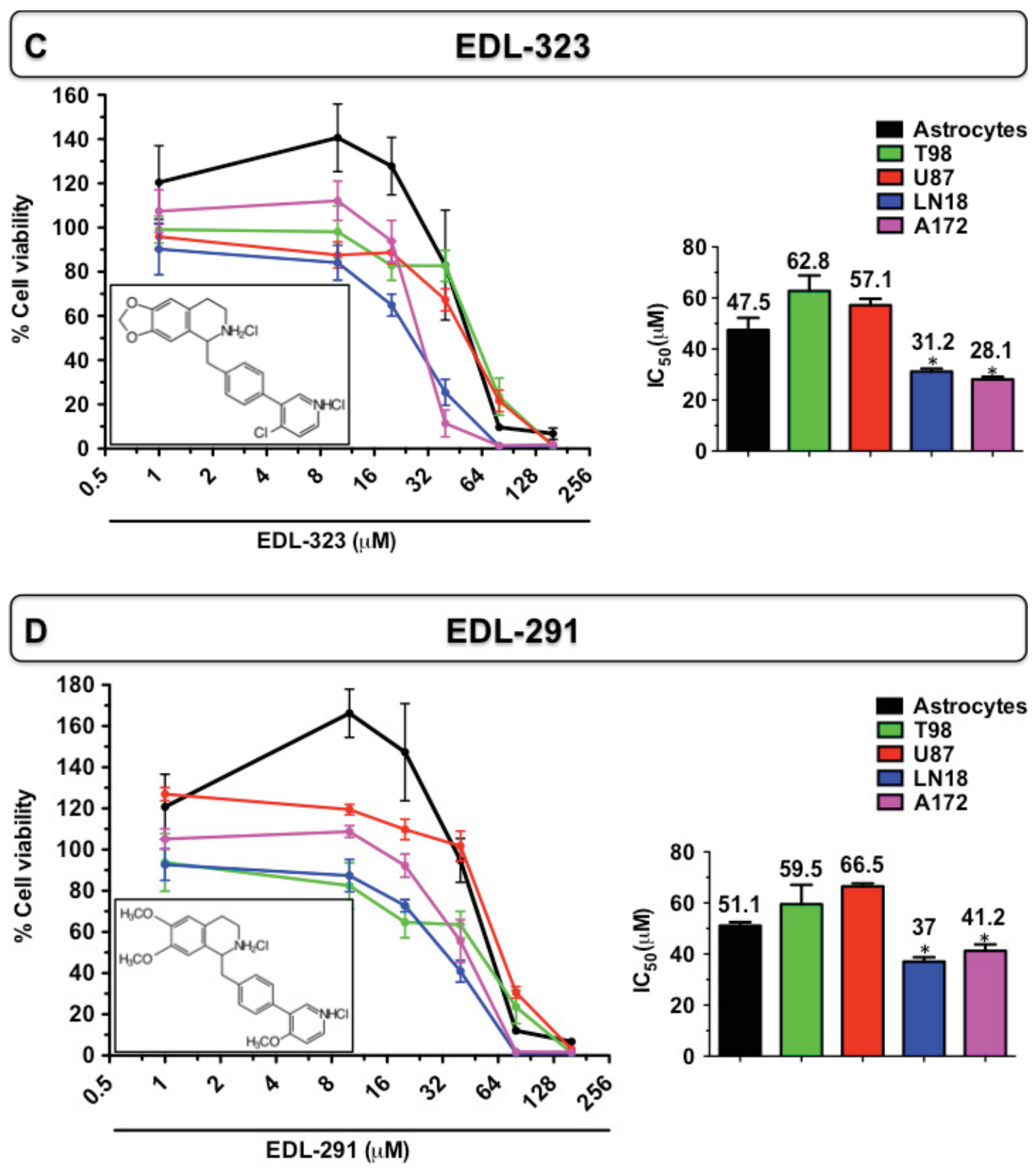

Figure 4-2. Continued. 

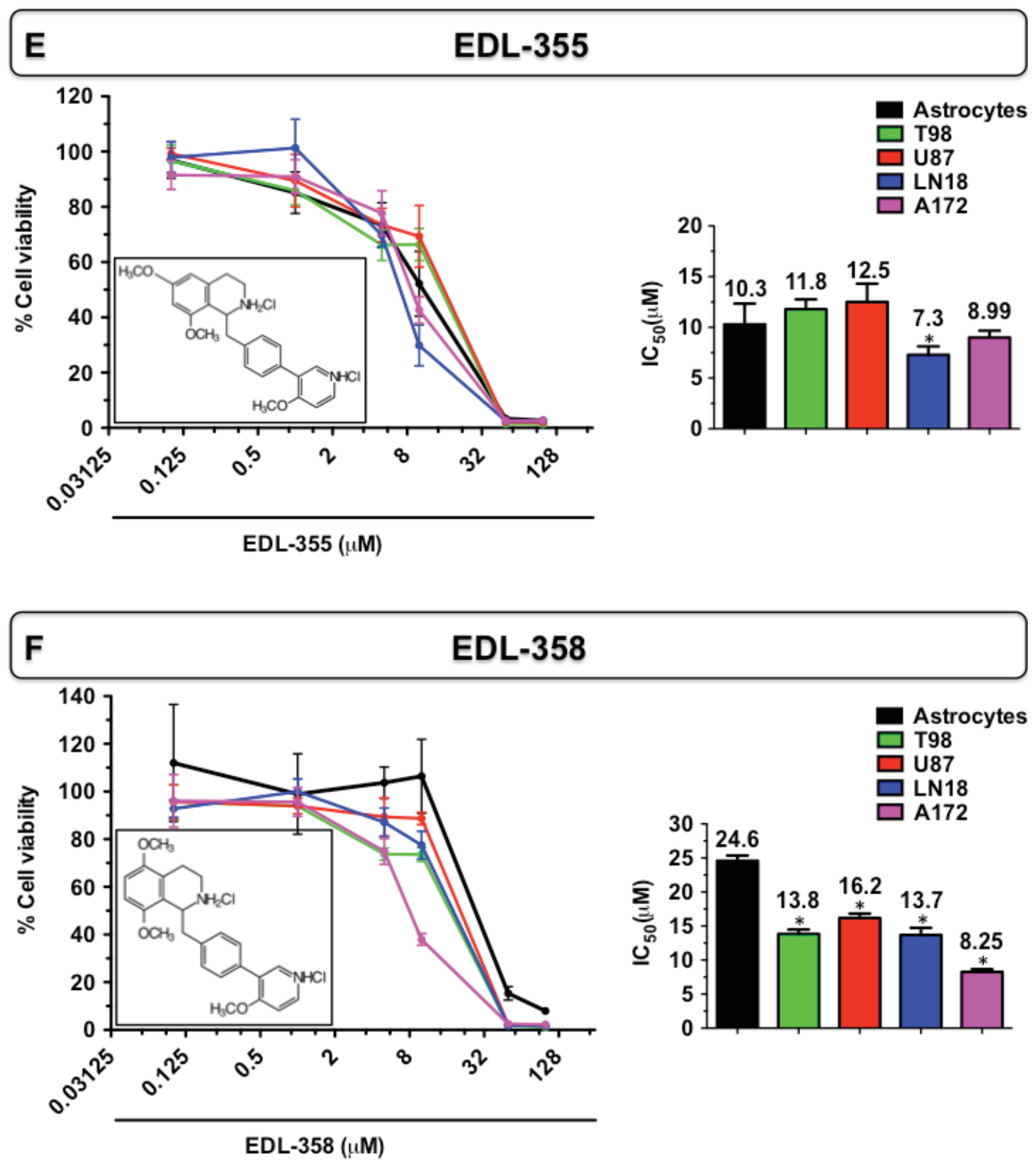

Figure 4-2. Continued. 

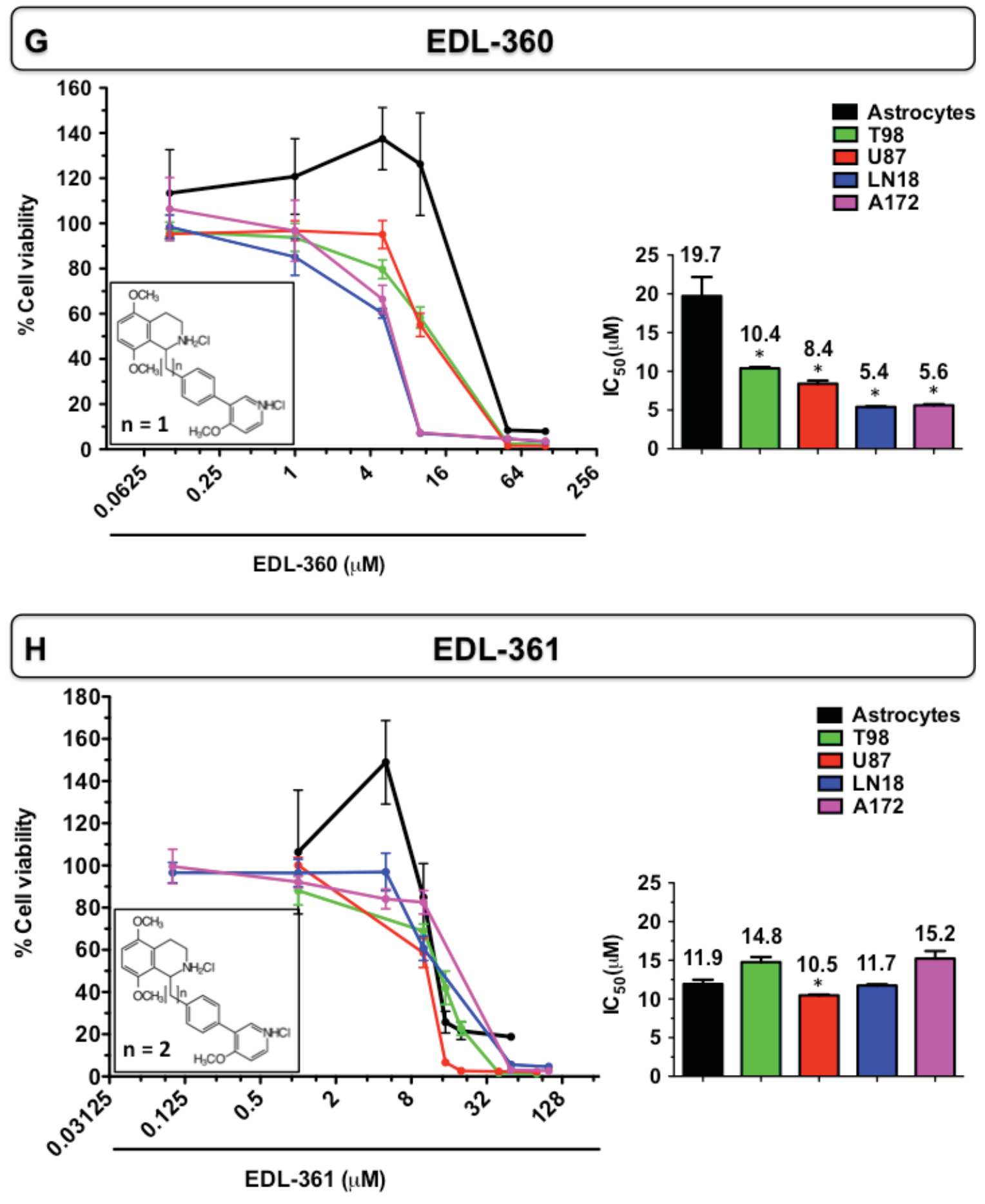

Figure 4-2. Continued. 
Figure 4-3. The effect of chromene analogs on the cell viability of glioma and astrocytes.

A, LN18, U87 and A172 had significantly lower $\mathrm{IC}_{50}$ values for SP-6-19 compared to normal astrocytes. B, SP-6-21 was less toxic compared to SP-6-19. However, the observed cytotoxicity remained in the submicromolar range in glioma cell lines. $\mathbf{C}$, SP-6-23 exhibited even less toxicity compared to SP-6-21. D, Chromene analog, SP-6-25, exerts anti-glioma activity in micromolar range. Interestingly, astrocytes were quite insensitive to its cytotoxicity. E, SP-6-27 showed an enhanced activity and selectivity. All tested glioma cell lines had significantly lower $\mathrm{IC}_{50}$ values compared to normal astrocytes. F, SP-6-35 appeared to be less active compared to SP-6-27 and it selectively induced cell death in T98 and LN18 compared to U87 and A172. G, Similarly, SP-6-37 exhibited a selective toxicity in LN18 and A172 compared to T98 and U87. Up to $100 \mu \mathrm{M}$ of SP-6-37 did not cause significant cell death in astrocytes. $\mathbf{H}$, There is a large decrease in SP-6-41 activity compare to any other chromene compound tested thus far. However, it was not quite toxic to astrocytes compared to glioma cell lines. I, SP-6-43 showed a slightly higher anti-glioma effect compared to SP-6-41. MTT cell viability using escalated doses of the test compounds (left panels). Bar graphs comparing the $\mathrm{IC}_{50}$ data for normal mouse astrocytes and human glioma cell lines (right panels). Bars represent the means; whiskers represent the standard deviation $( \pm),{ }^{*} p<$ 0.05. Adapted with permission from Patil R, Hosni-Ahmed A, Jones T, Patil S, Asres L, et al. (2014) Synthesis and In Vitro Evaluation of Novel 1,2,3,4-Tetrahydroisoquinoline Derivatives as Potent Antiglioma Agents. Anticancer Agents Med Chem (In Press). 

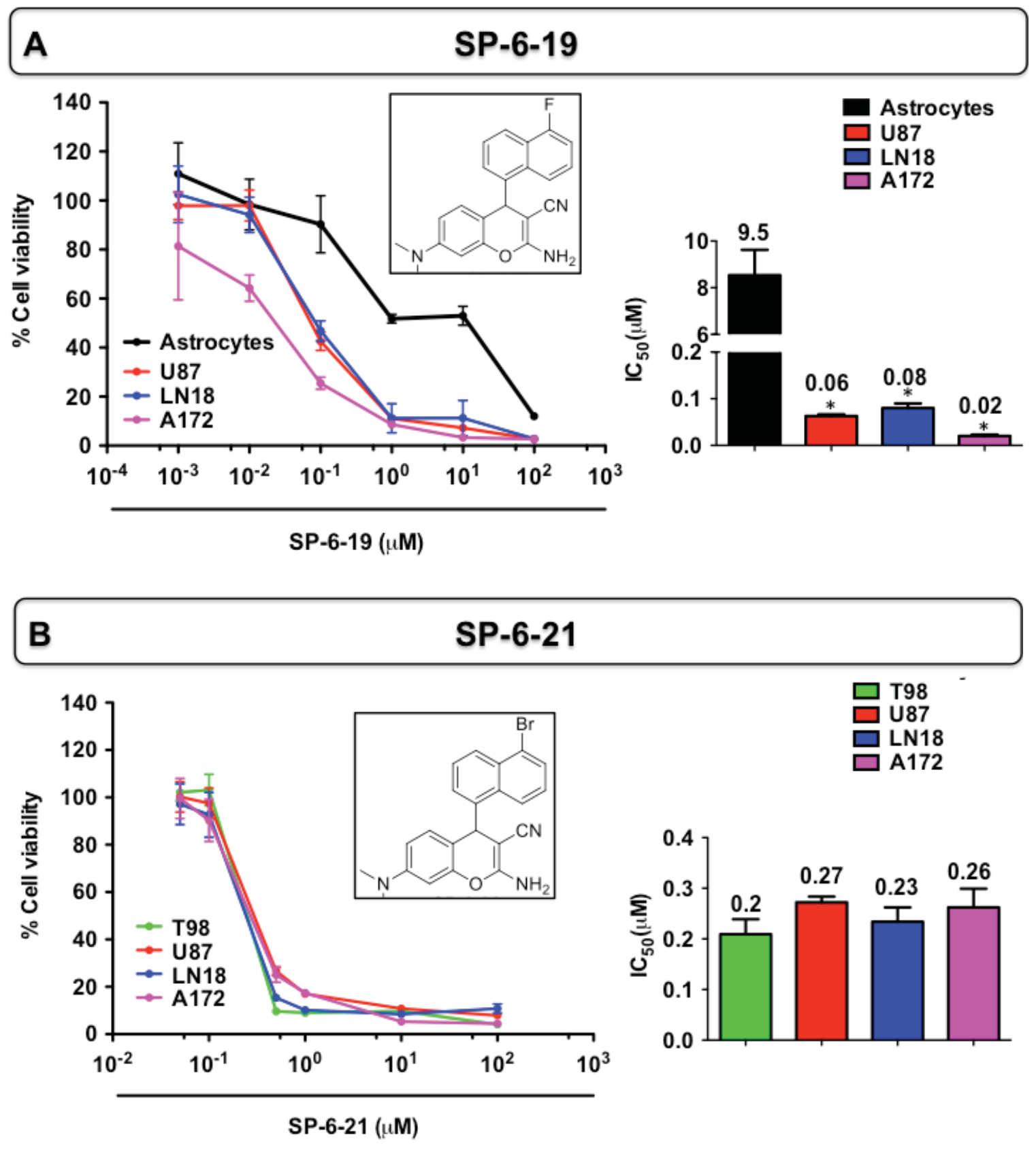

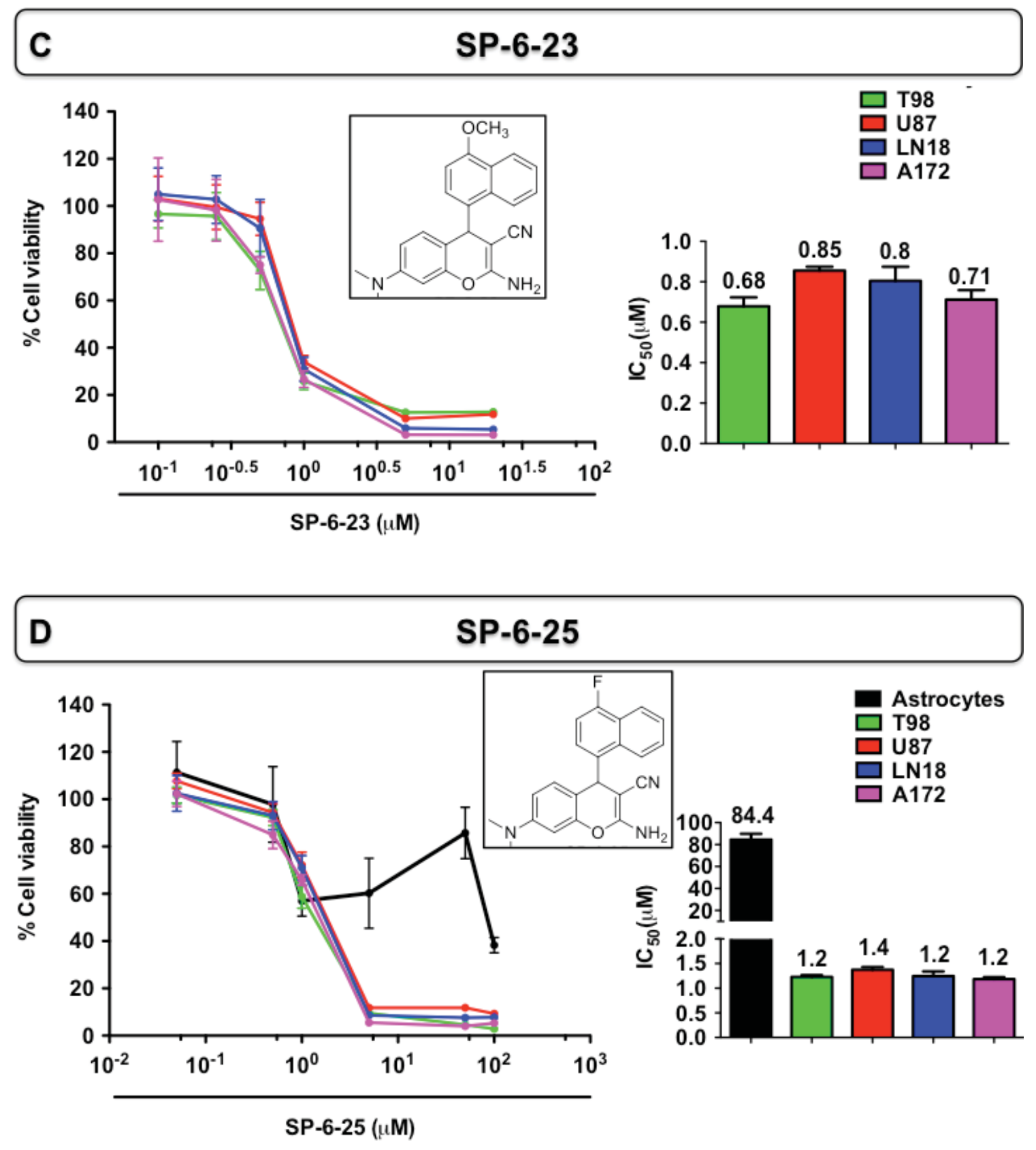

Figure 4-3. Continued. 

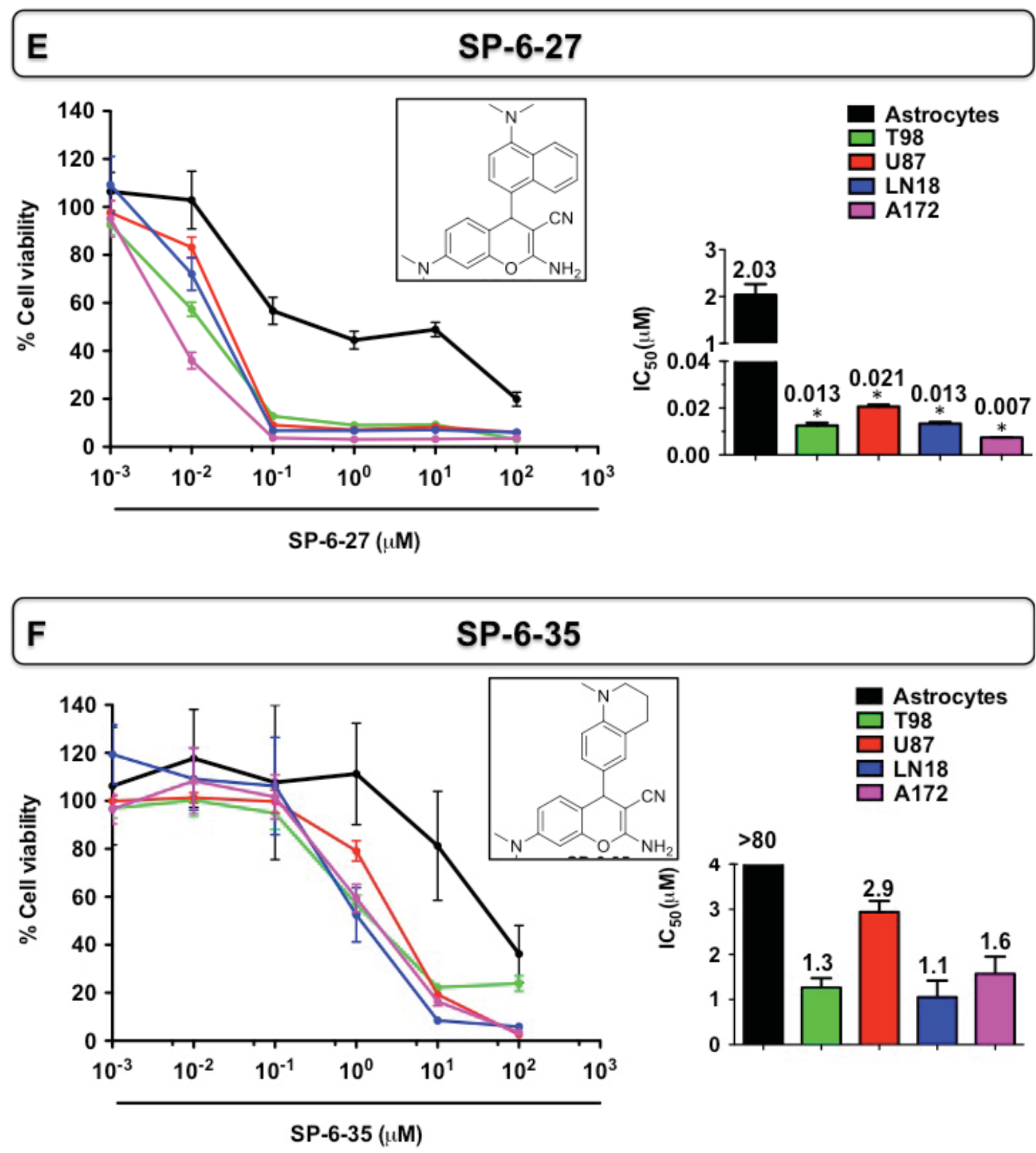

Figure 4-3. Continued. 

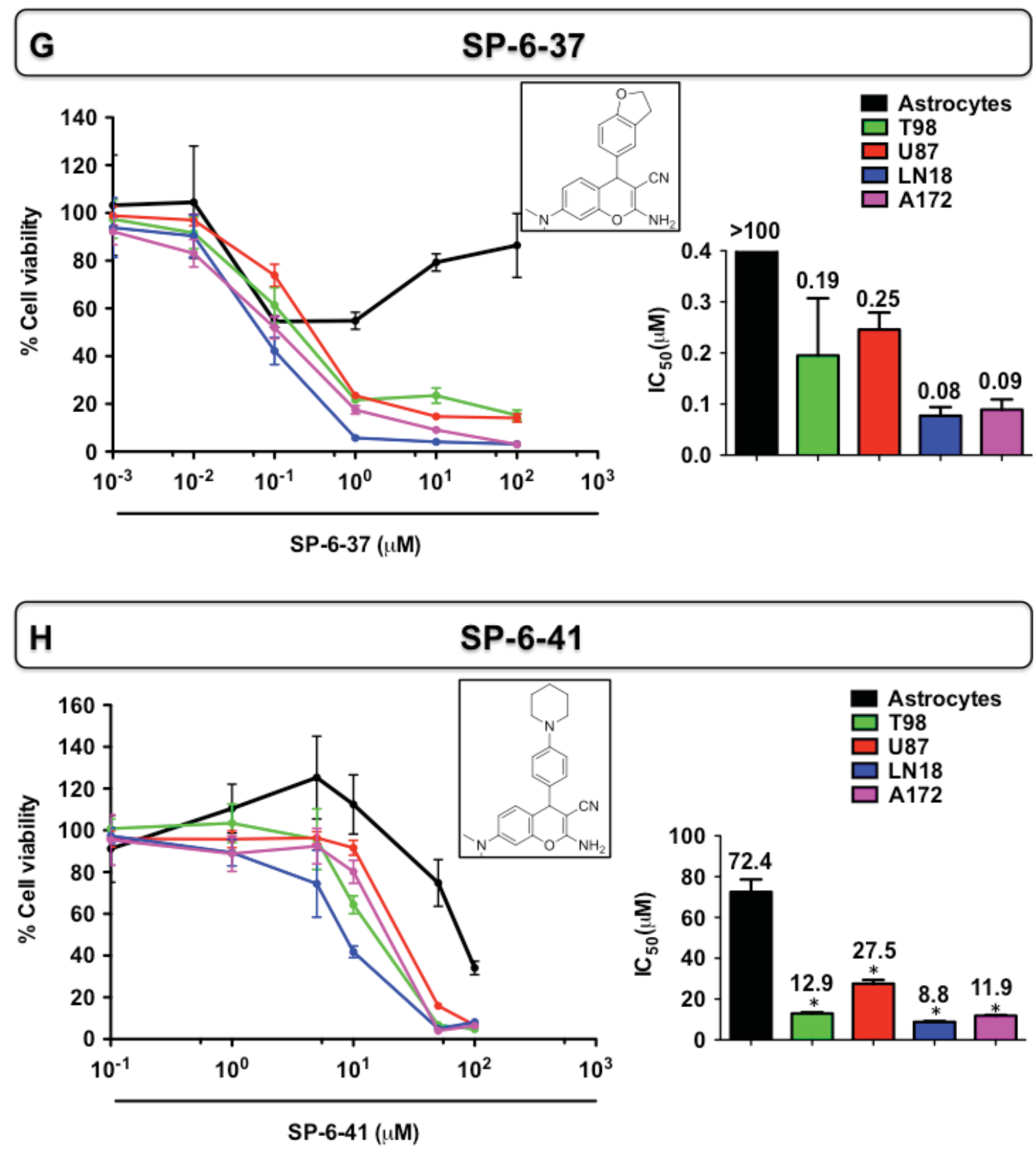

Figure 4-3. Continued. 


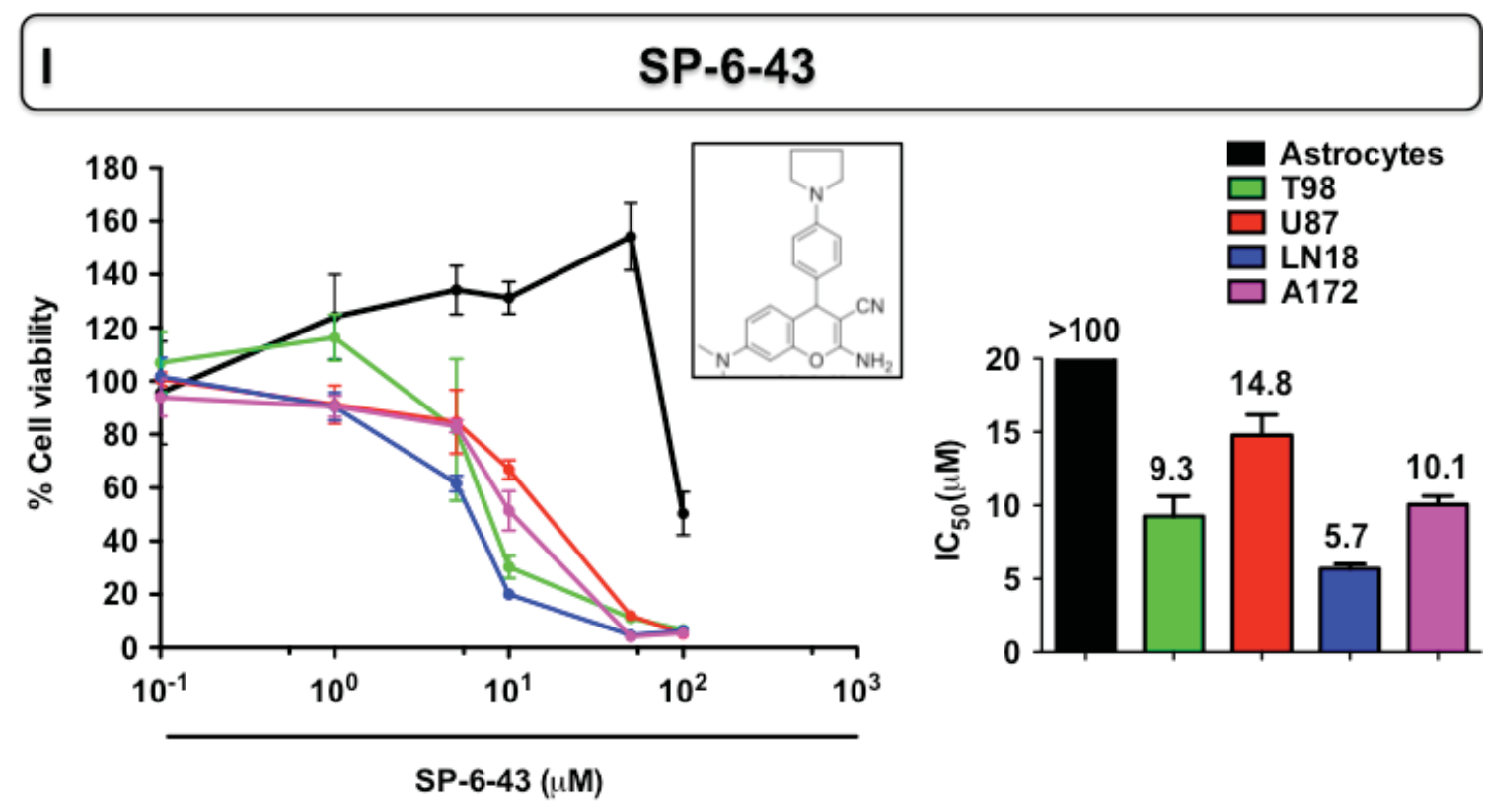

Figure 4-3. Continued. 
0.05). In contrast, SP-6-35 had a much lower activity compared to SP-6-27 (Figure 4-3F). SP-6-35 selectively induced cell death in T98 and LN18 cells compared to U87 and $\mathrm{A} 172$ cells. The $\mathrm{IC}_{50}$ values ranged from 1-3 $\mu \mathrm{M}$ in glioma cell lines, and it was approximately $90 \mu \mathrm{M}$ in normal mouse astrocytes. Similarly, SP-6-37 exhibited selective toxicity in LN18 and A172 compared to T98 and U87 cells (Figure 4-3G). Up to $100 \mu \mathrm{M}$ of SP-6-37 did not cause significant cell death in astrocytes. There is a large decrease in SP-6-41 and SP-6-43 activity compared to any other chromene compounds tested thus far (Figure 4-3H and Figure 4-3I). However, it was not as quite toxic in astrocytes as compared to glioma cell lines. Astrocytes had a higher viability at 1, 5, 10, 50 and 100 $\mu \mathrm{M}$ of SP-6-41 and the $\mathbf{I C}_{\mathbf{5 0}}$ in astrocytes was significantly higher than on glioma cell lines. Finally, SP-6-43 showed a slightly higher anti-glioma effect compared to SP-6-41. The $\mathrm{IC}_{50}$ value for glioma cell lines ranged from 6 to $15 \mu \mathrm{M}$, whereas in astrocytes it was $>100 \mu \mathrm{M}$.

\section{Effect of EDL-360 in Human Glioma Xenograft Mouse Model}

Based on in vitro screening results, EDL-360 was found to be the most potent THIQ analog. To assess its anti-glioma activity in vivo, mice bearing U87 glioma xenografts were treated with a daily single dose of EDL-360, and tumor engraftment examined by caliper and xenogen animal imaging. For bioluminescence imaging, U87 glioma cell line was transduced with the pLenti-Luc-mKate-pgk-puro viral vector, and the cells were grown in puromycin to select stably transfected cells. Luciferase activity was measured in $U 87^{\text {luc }}$ and $U 87^{\text {mock }}$ cultures using dual-luciferase reporter assay. Mean luciferase luminescence was $1.79 \times 10^{7} / 8.3 \times 10^{5}$ cells $\left( \pm 6.4 \times 10^{5}\right)$ and $173 / 7.8 \times 10^{5}$ cells $( \pm 19)$ relative light units (RLU) in $U 87^{\text {luc }}$ and $U 87^{\text {mock }}$, respectively. To generate the glioma mouse model, U87 $7^{\text {luc }}$ cells were injected subcutaneously into the right flank of 4-6 week old BALBc (NSG) mice. Once the tumor became palpable the mice were randomly assigned into groups of 5 mice. The treated group received $5 \mu \mathrm{M}$ of EDL-360 daily, whereas the control group did not receive any treatment. Mice were monitored daily for any signs or symptoms of drug related toxicity. In addition, mice were weighed twice a week. There was no sign of toxicity or weight loss in the EDL-360 treated group compared to the control mice (Figure 4-4A).

Tumor dimensions (width, length and height) were obtained using a digital caliper to determine tumor volume $\left(\mathrm{mm}^{3}\right)$. Tumor volume of each mouse was normalized to the initial volume before starting EDL-360 treatment (day 0). A slight attenuation of tumor growth was observed as early as 3 days post-treatment $(\mathrm{P}=0.077)$ (Figure 4-4B). The tumors in the EDL-360 treated mice were significantly smaller compared to control mice after 7,10 and 14 days of treatment $(\mathrm{P}=0.03,0.02$, and 0.02 respectively). The difference in tumor size between EDL-360 treated tumors and the controls increased with time of treatment. For instance, 3 days after treatment the EDL-360 treated tumors were $1 / 3$ the size of the control group (Mean $=1.5$ vs 5 normalized tumor volume). After 14 days of treatment, the treated tumors were 1/6 the size of control group (Mean $=12.5 \mathrm{vs}$ 74 normalized tumor volume). In addition, non-invasive live imaging was employed to evaluate tumor growth in EDL-360 and control mice. Bioluminescence signal showed an 
Figure 4-4. Intratumoral treatment with EDL-360 in human glioma xenograft mouse model.

U87 $7^{\text {luc }}$ cells were injected SQ into the right flank of the mice. Once the tumor become palpable the mice were randomly placed in the study. A, Mouse weight was obtained twice a week. There was no sign of toxicity or weight loss in EDL-360 treated group compared to the control mice. B, Tumor dimensions (width, length and height) were obtained using digital caliper to determine tumor volume. The tumors of the control mice were growing significantly faster compared to EDL-360 treated mice $(\mathrm{P}<0.03)$. C, Bioluminescence signal from control and treated mice showed an obvious decrease in tumor viable cells after 17 days of treatment. D, Quantification of bioluminescence signals showed a continuous tumor growth in the control mice. In addition, there was a significant decrease in tumor growth in the drug treated mice compared to the controls ( $\mathrm{P}$ $<0.03)$. Bars represent the means; whiskers represent the standard deviation $( \pm)$; ${ }^{*} \mathrm{p}<$ 0.05 . 

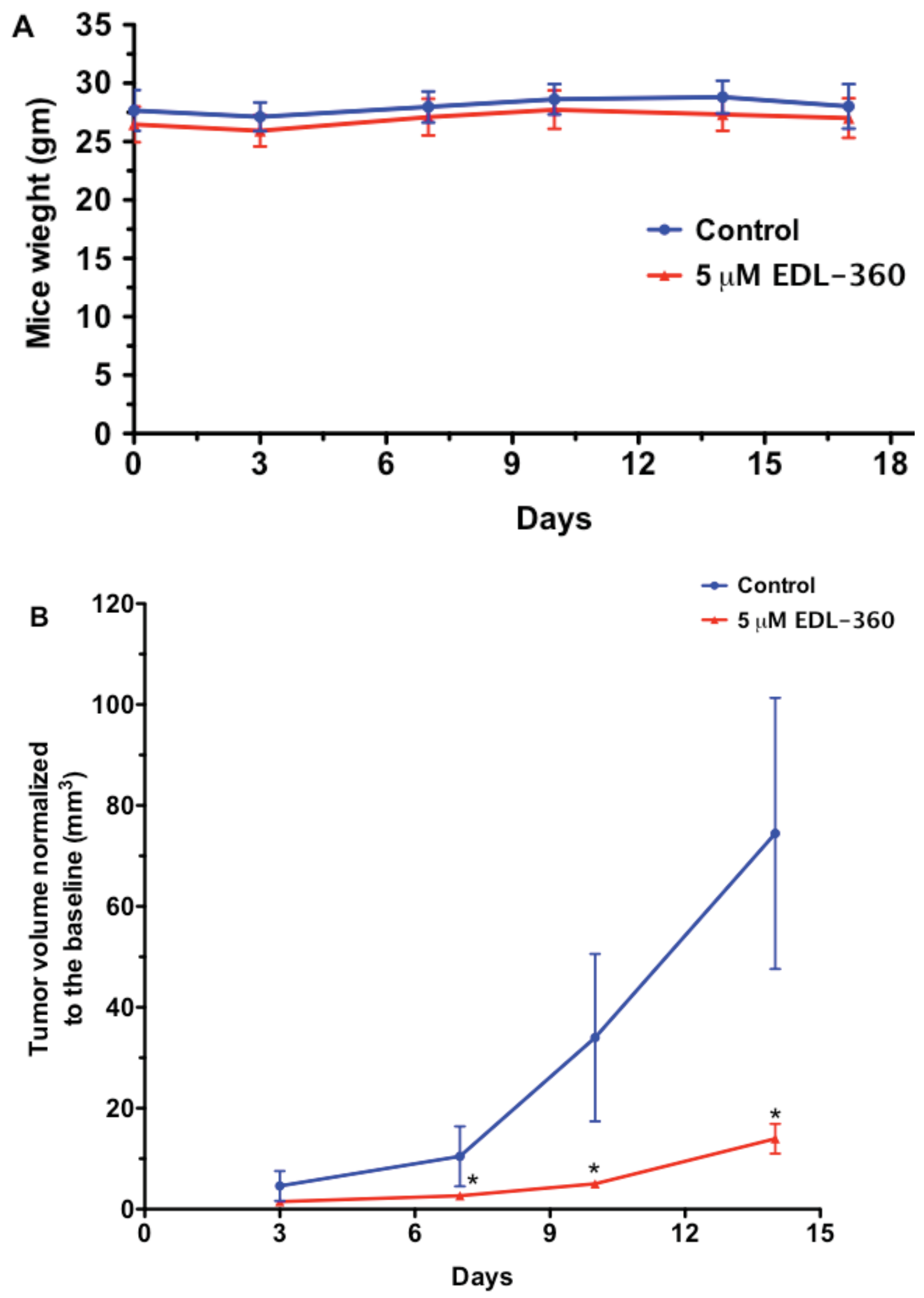

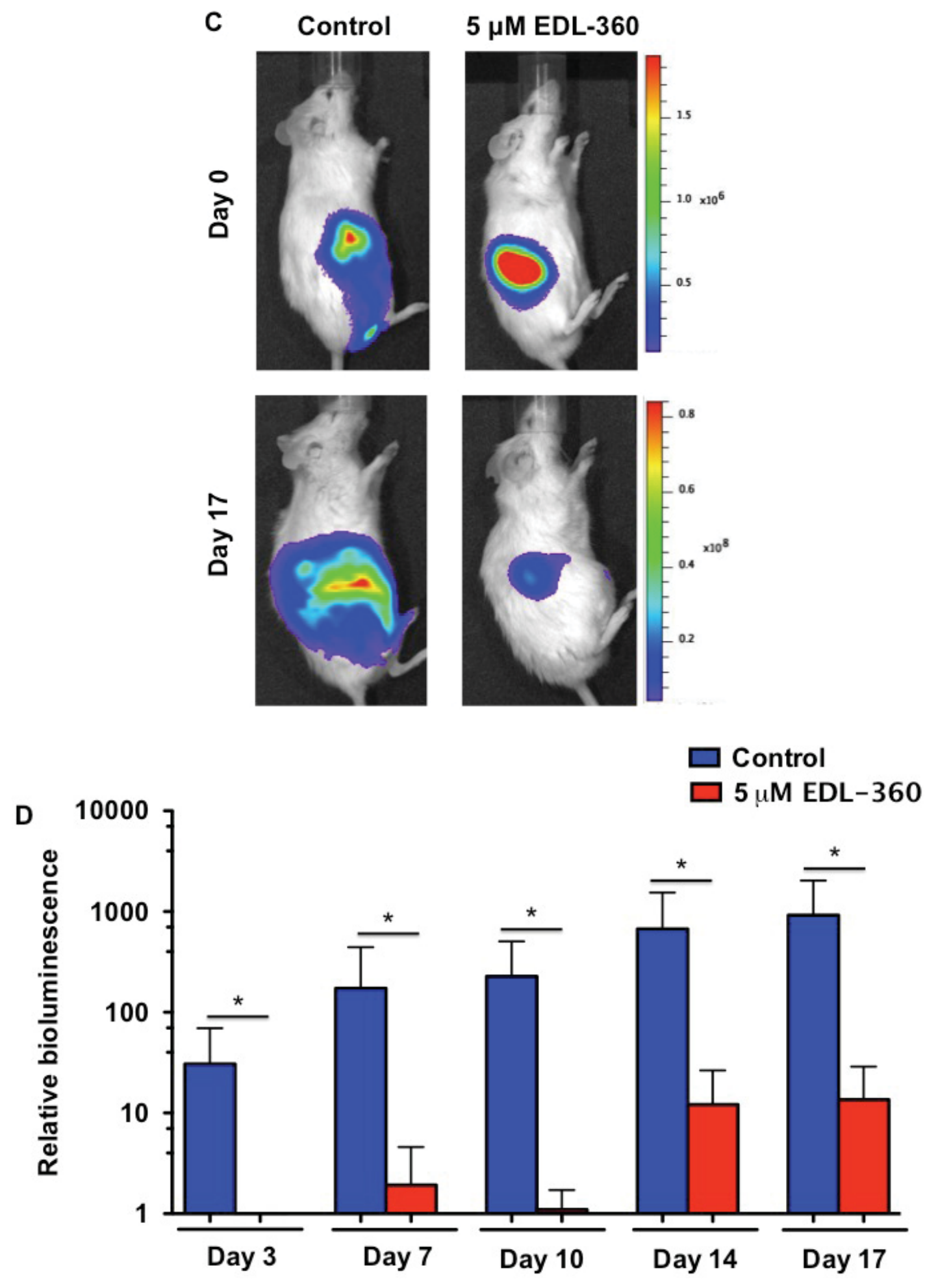

Figure 4-4. Continued. 
obvious decrease in tumor viable cells at 17 days of treatment compared to the controls (Figure 4-4C). Quantification of photons showed a continuous tumor growth in the control mice (Figure 4-4D). In addition, EDL-360 treated tumors had a significantly lower growth rate compared to the controls $(\mathrm{P}<0.03)$.

\section{Effect of SP-6-27 in Human Glioma Xenograft Mouse Model}

SP-6-27 was the most potent chromene analog in glioma cell lines in vitro and its activity was assessed in U87 $7^{\text {luc }}$ human glioma xenograft mouse model. Glioma-bearing mice were randomly divided into 3 groups. Treated groups received $100 \mu \mathrm{L}$ daily dose of 0.2 or $2 \mu \mathrm{M}$ SP-6-27/0.1\% acetic acid, whereas the control group received $100 \mu \mathrm{L}$ of $0.1 \%$ acetic acid. Mice were monitored daily for any signs or symptoms of drug related toxicity. Total body weight, tumor volume, and bioluminescence imaging were obtained twice a week during the study period. However, it appeared that the low $\mathrm{pH}$ environment created by the use of acetic acid affected the bioluminescence signal [172]. Therefore, the bioluminescence imaging results were excluded from the study. We observed no signs of drug related toxicity in the treated groups. In addition, there was no weight loss in SP-6-27 or vehicle treated groups (Figure 4-5). Drug treated groups had a smaller tumor volume compared to the vehicle treated group. Mice receiving $2 \mu \mathrm{M} \mathrm{SP-6-27}$ had significantly smaller tumors after 4 and 11 days of treatment $(p=0.016)$. As expected, tumor size decreased in a dose dependent manner.

\section{Discussion}

The THIQ analog, EDL-155, was found to be the most potent analog in rat glioma model [165]. When compared to other anti-cancer agents such as 5-fluorouracil, BCNU and melphalan, EDL-155 most effectively induced glioma cell death. In agreement with previous studies, EDL-155 was found to be very potent. The $\mathrm{IC}_{50}$ values ranged from 6-15 $\mu \mathrm{M}$ depending on the glioma cell line [165]. In the current study, we showed that all the THIQ analogs tested were more potent than TMZ, a standard chemotherapeutic agents for treating glioma. Interestingly, LN18 cells were resistant to the cytotoxic action of TMZ $(\sim 390 \mu \mathrm{M})$ and in our study EDL-155 was found to be highly toxic to LN18 cultures $(\sim 4 \mu \mathrm{M})$ (Figure 4-2B). This could indicate a potential use of our THIQ derivatives in combination with TMZ for TMZ-resistant gliomas. Further modifications in the $\mathrm{A}$ and $\mathrm{D}$ rings were made in attempt to improve the toxicity as well as the selectivity. The activity was lost in EDL-232 and partially recovered by adding 2 ortho-methoxy groups in A ring. Consequently, structure activity relationship showed that changing the methoxy groups to meta- (EDL-355) and para-position (EDL-358) (Figure 4-2E and Figure 4-2F), resulted in an obvious improvement of the cytotoxicity (EDL-355) as well as selectivity (EDL-358). Additionally, we lengthened the carbon linker between biphenyl and THI ring to 2-carbon atoms (EDL-360), which resulted in a further increase in the potency of the compound while maintaining low toxicity in astrocytes. However, adding an additional carbon atom (EDL-361) led to decreased in the cytotoxicity (Figure 4-2H). In conclusion, in vitro screening suggested that EDL-360 
Figure 4-5. Intratumoral treatment with SP-6-27 in human glioma xenograft mouse model.

The mice were divided in 3 groups, 5 mice/group. Control mice were injected daily with $0.1 \%$ acetic acid and treated groups were injected daily with $0.2 \mu \mathrm{M}$ or $2 \mu \mathrm{M}$ SP-6-27 in $0.1 \%$ acetic acid. A, Mice weight was obtained twice a week. There was no sign of toxicity or weight loss in SP-6-27 treated group compared to the vehicle control mice. B, Tumor dimensions (width, length and height) were obtained using digital caliper to determine tumor volume. Tumors in the SP-6-27 treated groups were growing at a slower rate compared to the control mice. Mice that received $2 \mu \mathrm{M}$ SP-6-27 had significantly smaller tumors after 4 and 11 days of treatment $(\mathrm{p}=0.016)$. Bars represent the means; whiskers represent the standard deviation $( \pm) ; * p<0.05$. 

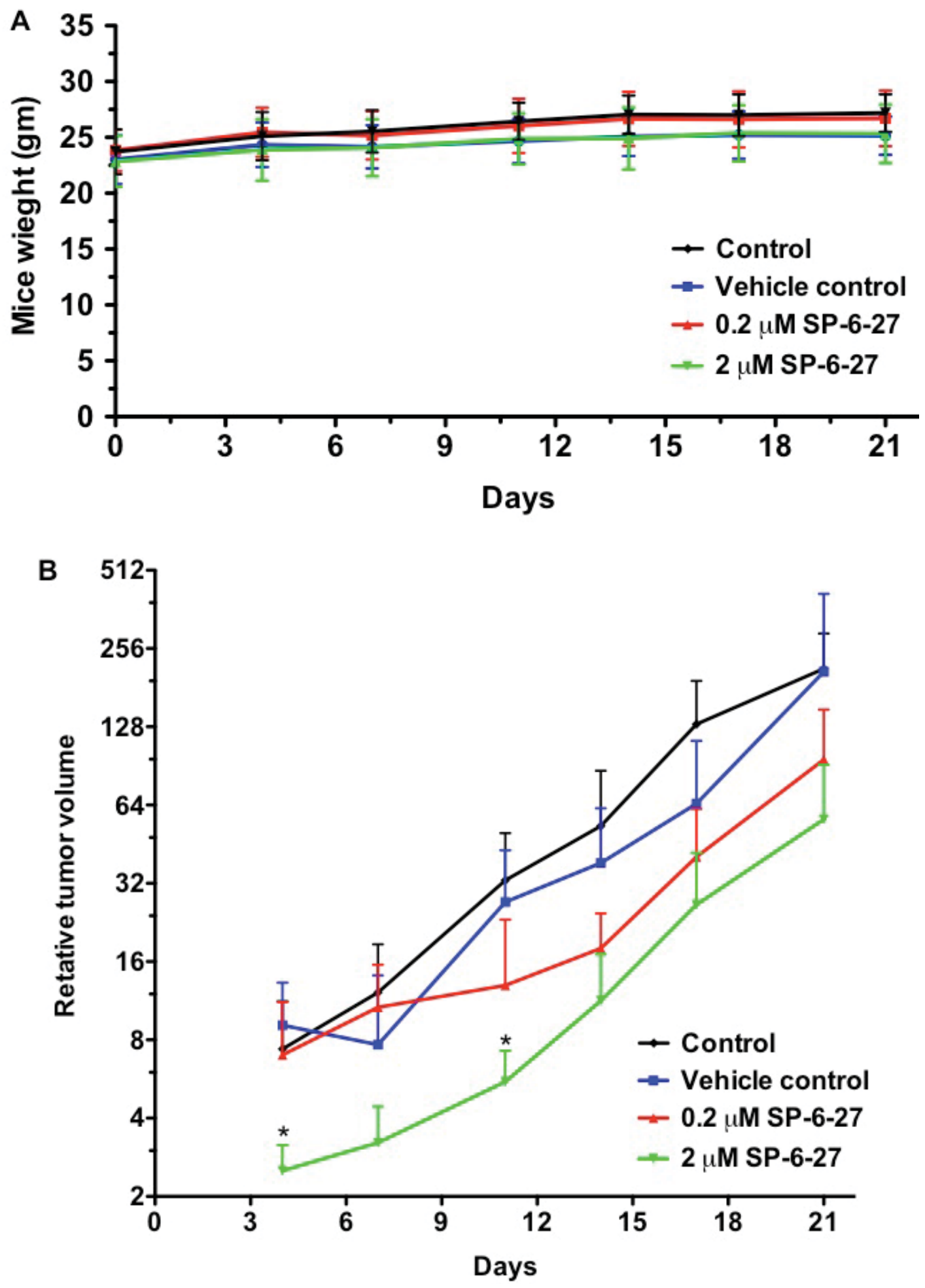
is the most potent THIQ analog. Interestingly, it appears that EDL-360 exhibited a selective toxicity in LN18 and A172 cultures when compared to T98 and U87 cultures raising the potential of use of this analog in the clinical setting for gliomas that are resistant to $\mathrm{TMZ}$.

Chromene analogs were a second group of compounds that were assessed for their potency against glioma. Chromenes are analogs of podophyllotoxin, a natural plant product, that have been isolated from Podophyllum species [169]. Early clinical trials revealed that podophyllotoxins induce cell death by inhibiting microtubule assembly [169]. In addition podophyllotoxins can inhibit purine biosynthesis and its incorporation into the RNA as well as decreasing the activity of mitochondrial enzymes such as cytochrome oxidase and succinoxidase. More importantly, podophyllotoxin derivatives are potent inhibitors of DNA topoisomerase II and hence induce cell death [173]. Consequently, several studies reported these derivatives as potent anti-cancer agents [174-177]. In vitro screening of various chromene analogs revealed that SP-6-25, SP-6-35, SP-6-41 and SP-6-43 are the least potent analogs (Figure 4-3). The $\mathrm{IC}_{50}$ of these compounds was in micromolar range. However, SP-6-27 followed by SP-6-19 were the most potent chromene analogs tested in the current study. More importantly, these compounds demonstrated very low activity in normal astrocytes compared to glioma cell lines, implying a potential benefit with least toxicity. Based on structure activity relationship, it appears that the substitution of electron withdrawal atom on D-ring and/or electron donor atom on C-ring resulted in an improvement of chromene potency [168].

Based on our in vitro drug screening findings, we selected the most potent THIQ (EDL-360) and chromene (SP-6-27) analogs for in vivo evaluation in human glioma xenograft model to address this question. EDL-360 caused partial regression in tumor size (Figure 4-4B) without causing a notable toxicity in the treated mice (Figure 4-4A). Tumor size was determined by digital caliper measurement and live animal bioluminescence imaging (Figure 4-4C and Figure 4-4D). However, it is still unclear whether EDL-360 could penetrate the BBB, and would exert a similar anti-cancer effect when the tumors were grown intracranially. Previous studies showed that EDL-155 could penetrate the BBB, with $1.4 \%$ brain to plasma exposure ratio [178]. However, it is not possible to expect that EDL-360 would exhibit good penetrance across the BBB as well. Slight variation in the chemical structure could lead to great changes in the biological responses. Similarly, tumor-bearing mice that were treated with SP-6-27 did not lose weight or showed signs of toxicity (Figure 4-5A). Additionally, SP-6-27 treatment caused a partial regression in tumor size (Figure 4-5B) similar to EDL-360 treated tumors. However, treatment with the vehicle (acetic acid) resulted in in a slight decrease in tumor growth, which in part could mask the effect of SP-6-27. In conclusion, EDL-360 and SP-6-27 have potential as anti-cancer agents. However, the limited solubility of SP-6-27 in aqueous solutions may be a limiting factor. Further investigations are required to test the anti-cancer activity of these small molecules in pre-clinical glioma mouse models. 


\section{CHAPTER 5. DISCUSSION AND CONCLUDING REMARKS}

Over the past 10 years, despite improvement in diagnosis and therapies for cancer, the survival rate for high-grade glioma patients remains low. In the current study, we investigated the effects of corticosteroids and thiopurines in the brain because they have been associated with increased resistance to chemotherapy or gliomagenesis, respectively.

\section{Corticosteroids Might Reduce the Efficacy of Chemotherapy and Radiotherapy}

Vasogenic edema develops in aggressive gliomas and corticosteroids are the first clinician choice to alleviate the neurological symptoms $[9,72]$. Despite the intensive use of Dex in the clinic, its effect on glioma is not precisely identified. Whether Dex has anti-proliferative or anti-neoplastic properties, or whether it stimulates cell growth has been a long debate in the literature $[35,36,76,77]$. We have found that Dex treatment decreases tumor cell proliferation as shown by PCNA and Ki67 IHC staining and hence it exhibits an anti-proliferative effect. Since cancer therapeutics often target rapidly growing cells, it is quite possible that Dex might compromise the responsiveness of tumor cells to chemotherapy and/or radiotherapy via inhibiting tumor cell growth. Indeed, several studies suggested that Dex might compromise the responsiveness of tumor cells to chemotherapy and/or radiotherapy[37,92,93]. Pre-treatment with Dex induces Bcl-xL expression and inhibited cell death induced by staurosporine, camptothecin and etoposide in astrocytoma cultures [93]. In breast and ovarian xenograft animal model, pretreatment with $1 \mathrm{mg} / \mathrm{kg}$ of Dexwas found to reduce the anti-cancer action of paclitaxel by 20-25\% [179]. Such findings highlight the desperate need to understand the exact effect of Dex on tumor cells especially for patients undergoing chemotherapy or radiotherapy concurrently with corticosteroids, and raises the concern that Dex treatment should not be combined with DNA damaging agents such as chemo- and radio-therapy.

\section{Corticosteroids Decrease Microglial Cell Number and M2-Like Microglial Cells}

The mechanism of how Dex alleviates cerebral edema is mostly unknown. However, it has been proposed that Dex might improve peritumoral edema through its anti-inflammatory effect or by inhibiting microglial cells proliferation and pro-inflammatory cytokine production $[68,73]$. In rat glioma, DEX was found to decrease microglial cell proliferations infiltration into the tumor [73]. In the current study, we found that Dex decreases microglial cell content in tumor sections as confirmed by anti-Iba-1 primary antibody in PDGF driven glioma mouse model. (Figure 2-6A). However, two different phenotypes of microglial cells exists; M1 and M2. M1-like microglial cells are known to possess pro-inflammatory properties. In contrast, various studies suggested that tumor-associated microglia could support the tumor proliferation and growth, and possess an M2-like anti-inflammatory phenotype [78,97,98]. In 
agreement with previous findings, we found that tumor associated microglia exhibit an M2-phenotype in a PDGF induced-glioma model (Figure 2-7). This implies that inflammatory microglial cells may not play a major role in edema formation, and Dex might alleviate peritumoral edema by a mechanism other than inhibiting the M1-pro-inflammatory microglial cells.

Several studies found a decrease in the production of several pro-inflammatory cytokines such as COX2, NO, IFN $\gamma$ and TNF $\alpha$ in lipopolysaccharide induced upon Dex treatment [180-182]. However, lipopolysaccharide induces the classical activation of microglial cells to M1-pro-inflammatory phenotype and it is well defined that microglial cell exhibit M2-pro-tumor phenotype in tumor microenvironment $[78,86]$. To our knowledge, this is the first study that investigates the effect of Dex on the gene expression of M2-like microglial cells using a clinically relevant glioma model. We found a shift in the microglia phenotype towards less M2 (MMP9 and IL-1ra) in both in vitro and in vivo samples upon Dex treatment. MMP9 is a member of matrix metalloproteinases family, which is produced primarily by immune cells such as macrophages/microglias and neutrophils [101]. MMP9 plays a pivotal role in tumor progression and invasion [97]. MMP9 deficient mice have less lung metastases in both melanoma and lung carcinoma animal models [102]. More importantly, MMP9 plays an important role in angiogenesis and induction of cell proliferation [101,103,104]. It is plausible that targeting M2-microglial cells could have a potential therapeutic benefit for treating glioma [183]. However, the use of corticosteroids in managing glioma is problematic, since it is associated with a decreased response to anti-cancer therapy. Consequently, we addressed the question of whether this decrease in M2-pro-tumor phenotype could play a role in tumor cell proliferation in Dex treated samples. Therefore, we investigated the anti-proliferative effect of Dex using primary glioma cultures. In contrast to our in vivo results, we could not detect a cell proliferation inhibition following Dex treatment. These contradicting findings highlight the difference between in vitro and in vivo conditions and this does not eliminate the possibility that microglial cell play an important role in supporting tumor cell proliferation.

Taken together, Dex might act against glioma favorable conditions in our mouse model. However, this effect might or might not be recapitulated when Dex is combined with DNA damaging agents such as ionizing radiation. Finally, the current study highlights the urgent need to find better therapeutic alternatives to corticosteroids that have less adverse effects and interfere less with anti-cancer therapy. Further studies should focus on identifying the mechanism of action by which Dex inhibits cell proliferation. In addition it is important to investigate whether Dex alleviates preitumoral edema by inhibiting the expression of VEGF and hence decreases the permeability of the BBB.

\section{Low TPMT Activity Leads to Increases in TG-Induced Toxicity}

Thiopurine drugs are commonly used in the treatment of cancer, autoimmune disorders, and transplantation. TPMT is a cytoplasmic enzyme that deactivates thiopurine 
drugs and a low TPMT phenotype can lead to life-threatening hematological toxicities when standard doses of thiopurines are given. The use of these agents has been linked with the development of secondary brain tumors in TPMT-deficient patients [42,47]. Astrocytomas are the most common brain tumor type that has been reported in patients after thiopurine therapy $[47,48,50,67]$. Unfortunately, not all patient populations that are treated with thiopurines are evaluated for their TPMT status prior to initiating thiopurine therapy; hence, the importance of TPMT to thiopurine-associated cancer risk is not clear. Since TPMT deficiency is relatively rare across populations, it is difficult to assess the importance of TPMT status to thiopurine therapy-related cancer risk. However, in a report by Relling et al. it was shown that whether patients developed a brain tumor as a late complication of post-antileukemic therapy depended on their TPMT status [47]. Patients who were determined to be at high risk for treatment relapse received prophylactic cranial irradiation concurrent with antimetabolite therapy; and those patients with a low TPMT had a surprisingly high incidence of brain tumors as a late complication. Indeed, ionizing radiation is a risk factor for brain tumorigenesis. However, in this report, patients with low TPMT had a $42.9 \%$ incidence of brain tumors compared to $15.8 \%$ for those with high TPMT. To our knowledge, the importance of TPMT status to thiopurine-associated phenotypes in the brain has not been investigated.

We found that low TPMT phenotype was associated with significantly higher sensitivity to thiopurine than a high TPMT phenotype. In agreement with previous studies, we found that thiopurines induced significantly more cell death and DNA damage (SSB and DSB) [156]. Interestingly, we found that low but not the complete absence of TPMT sensitized primary astrocytes to a higher degree of cytotoxicity and genotoxicity (i.e. IC50, SSBs and DSBs) after TG exposure. These results suggested that heterozygosity in TPMT may lead to the greatest genotoxic effects of thiopurine therapy. In part this could be explained by the induction of compensatory mechanism that deactivates thiopurines upon the complete loss of TPMT. The loss of TPMT could have led to the overexpression of xanthine oxidase, which deactivates thiopurine agents to thiouric acid. Interestingly, some studies suggest that children with TPMT heterozygosity develop brain tumors and other cancers more frequently than children with mutant TPMT [164]. Another possible explanation could be a defect in the mismatch repair (MMR) system's ability to recognize DNA-TG nucleotide lesions in TPMT mutant cultures. The MMR system is responsible for recognizing DNA mis-pairing caused by DNA-TG nucleotides and the subsequent signaling for cell death [160-163]. The latter possibility suggests that astroglial cells with a defective TPMT activity could have DNA lesions persisting in the genome that could potentially lead to mutagenesis and cancer. However, the frequency of heterozygous or mutant TPMT is very low making it is difficult to make any definitive conclusions $[47,164]$. Indeed the current study raises the concern about thiopurine toxicity not only in TPMT mutant individuals but also in TPMT heterozygous. Therefore, a routine assessment of TPMT activity might be required for patients receiving thiopurine agents.

In summary, chronic thiopurine therapy has been associated with the development of brain cancer and TPMT status has been linked to brain tumor risk. We found that TPMT is an important factor for thiopurine drug-induced cytotoxicity and genotoxicity in 
astroglial cells. Because of the limited availability of human astrocytes we used human glioma cell lines. Although glioma cells can exhibit a number of genetic alternations that could contribute to the phenotypes observed, our study shows that TPMT is indeed an important determinant of thiopurine toxicity. The observation that glioma cells can have variable degrees of TPMT protein activity and that low protein activity can cause cells to be more sensitive to TG may suggest that thiopurines may potentially have a therapeutic role in low TPMT expressing glial tumors. However, since thiopurine drugs are linked with secondary brain tumors, we do not recommend the use of these agents in brain tumor treatment. Furthermore, In vitro studies have shown that thiopurine drugs are associated with mutagenic events in a variety of cell lines [116,151].

Further studies are needed to understand the importance of TPMT in the brain when thiopurines are given. Whether these findings can be replicated in vivo and whether factors important to DNA repair mechanisms are responsible for the TG phenotypes described in this study should also be investigated. Finally, It would be interesting to investigate whether TPMT status influences the extent of TG nucleotide incorporation into DNA and damage recognition by the MMR system as well as whether ionizing irradiation alone or in combination with thiopurines results in similar findings.

\section{EDL-360 and SP-6-27 Exhibit Anti-Glioma Activity In Vitro and In Vivo}

Despite aggressive therapeutic approaches to treat glioma, there is no significant improvement in either the prognosis or the survival of patients. Therefore, there is an urgent need to find new therapeutic approaches to improve patients' survival and outcome. The current study focused on assessing the potential anti-cancer activity of two categories of small molecules; THIQ and chromenes.

The anti-cancer activity of our compounds has been tested in vitro using MTT cell viability assays. Since glioma is a heterogeneous tumor, the in vitro drug screening was performed in four human glioma cell lines (T98, U87, LN18 and A172). Although normal human astrocyte cultures may be more appropriate as a model of normal brain cells, normal murine astrocyte cultures were used due to their availability in our laboratory. Based on our in vitro screening, we identified EDL-360 and SP-6-27 as the most potent derivatives with lowest toxicity to normal stromal cells. Interestingly, it appears that EDL-360 exhibited a selective toxicity in LN18 and A172 cells when compared to T98 and U87 cells raising the potential use of this analog in the clinical setting for gliomas that are resistant to TMZ. SP-6-27 exhibits cytotoxicity in nanomolar range (IC50 $=7-21 \mathrm{nM}$ ). Tubulin polymerization inhibition could in part play a role in SP-6-27 induced toxicity [168]. However, inhibition of tubulin polymerization by SP-6-27 was weak and other mechanism of actions might play a major role in SP-6-27 induced toxicity.

The anti-cancer activity of EDL-360 or SP-6-27 was further tested in glioma-bearing mice generated by injecting human glioma $\mathrm{U} 87^{\text {luc }}$ cells subcutaneously in immunocompromised mice. Despite the fact that U87 cell produces glioma characterized 
by leaky BBB similar to human glioma, the tumor does not have infiltrative nature [19]. There was no sign of toxicity as indicated by the absence of weight loss in the treated groups (EDL-360 and SP-6-27) compared to the control mice. The tumors of EDL-360 treated mice were significantly smaller compared to control mice after. In contrast SP-6-27 treatment resulted in a partial regression of tumor size. However, it is still unclear whether EDL-360 or SP-6-27 could penetrate the BBB and exerts a similar toxicity when the tumors grown intracranially. Previous studies showed that EDL-155 (THIQ analog) could penetrate the BBB, with $1.4 \%$ brain to plasma exposure ratio [178]. However, it is possible to expect that EDL-360 would exhibit better penetration of the BBB since it is a methoxy rather that a penolic substituted compound. Slight variation in the chemical structure could lead to great changes in the biological responses. Similarly, SP-6-27 treatment caused a partial regression in tumor size. However, acetic acid treated tumors did not grow as fast as non-treated tumors and this in part masking the effect of SP-6-27. It is also plausible that the stromal cells provide growth support to the tumor cells, rendering SP-6-27 ineffective.

In conclusion, EDL-360 and SP-6-27 have potential as anti-cancer agents.

However, the limited solubility of SP-6-27 in aqueous solutions could be a limiting factor for its use. The synthesis of the corresponding sodium or phosphate salts of SP-6-27 may enhance its water solubility. Probing the structure activity relationship of EDL-360 may improve its anti-cancer activity. In addition, further investigations are required to test the anti-cancer activity of these small molecules in an intracranial xenograft model, which would allow tumor development in the native microenvironment of the brain, and thus allows more reliable assessment of anti-glioma novel therapies. 


\section{LIST OF REFERENCES}

1. Holland EC (2000) Glioblastoma multiforme: the terminator. Proc Natl Acad Sci U S A 97: 6242-6244.

2. Dolecek TA, Propp JM, Stroup NE, Kruchko C (2012) CBTRUS statistical report: primary brain and central nervous system tumors diagnosed in the United States in 2005-2009. Neuro Oncol 14 Suppl 5: v1-49.

3. Burnet N, Bulusu V, Jefferies S (2004) Management of primary brain tumours; Booth S, Bruera E, editors. Oxford: Oxford University Press. xii, 166 p. p.

4. Parkin DM, Whelan SL, Ferlay J, Teppo L, Thomas DB (2002) Cancer incidence in five continents. Volume VIII. IARC Sci Publ: 1-781.

5. Bondy ML, Scheurer ME, Malmer B, Barnholtz-Sloan JS, Davis FG, et al. (2008) Brain tumor epidemiology: consensus from the Brain Tumor Epidemiology Consortium. Cancer 113: 1953-1968.

6. DeAngelis LM (2001) Brain tumors. N Engl J Med 344: 114-123.

7. Saul TG, Ducker TB (1982) Effect of intracranial pressure monitoring and aggressive treatment on mortality in severe head injury. J Neurosurg 56: 498-503.

8. Hildebrand J, Amoura Z, Baumert B (2003) Management of brain tumor edema. EANO-Guideline-Discussion peritumoral edema. 1-9 p.

9. Kaal EC, Vecht CJ (2004) The management of brain edema in brain tumors. Curr Opin Oncol 16: 593-600.

10. Koehler PJ (1995) Use of corticosteroids in neuro-oncology. Anticancer Drugs 6: 19-33.

11. Hsieh J (2009) Computed Tomography: Principles, Design, Artifacts, and Recent Advances. Bellingham, Washington USA: SPIE Press.

12. Chang AE, Matory YL, Dwyer AJ, Hill SC, Girton ME, et al. (1987) Magnetic resonance imaging versus computed tomography in the evaluation of soft tissue tumors of the extremities. Ann Surg 205: 340-348.

13. Bizzi A, De Stefano N, Gullapalli R, Lin D (2010) Clinical MR spectroscopy: techniques and applications. Cambridge, UK: Cambridge University Press. 1-256 p.

14. Preul MC, Caramanos Z, Collins DL, Villemure JG, Leblanc R, et al. (1996) Accurate, noninvasive diagnosis of human brain tumors by using proton magnetic resonance spectroscopy. Nat Med 2: 323-325. 
15. Louis DN, Ohgaki H, Wiestler OD, Cavenee WK, Burger PC, et al. (2007) The 2007 WHO classification of tumours of the central nervous system. Acta Neuropathol 114: 97-109.

16. Scherer HJ The forms of growth in gliomas and their practical significance. Brain 63: $1-35$.

17. Louis DN, Holland EC, Cairncross JG (2001) Glioma classification: a molecular reappraisal. Am J Pathol 159: 779-786.

18. Verhaak RG, Hoadley KA, Purdom E, Wang V, Qi Y, et al. (2010) Integrated genomic analysis identifies clinically relevant subtypes of glioblastoma characterized by abnormalities in PDGFRA, IDH1, EGFR, and NF1. Cancer Cell 17: 98-110.

19. Patil SA, Hosni-Ahmed A, Jones TS, Patil R, Pfeffer LM, et al. (2013) Novel approaches to glioma drug design and drug screening. Expert Opin Drug Discov 8: 1135-1151.

20. Network CGAR (2008) Comprehensive genomic characterization defines human glioblastoma genes and core pathways. Nature 455: 1061-1068.

21. Pang BC, Wan WH, Lee CK, Khu KJ, Ng WH (2007) The role of surgery in high-grade glioma--is surgical resection justified? A review of the current knowledge. Ann Acad Med Singapore 36: 358-363.

22. Do V, Gebski V, Barton MB (2000) The effect of waiting for radiotherapy for grade III/IV gliomas. Radiother Oncol 57: 131-136.

23. Stupp R, Mason WP, van den Bent MJ, Weller M, Fisher B, et al. (2005) Radiotherapy plus concomitant and adjuvant temozolomide for glioblastoma. N Engl J Med 352: 987-996.

24. Jones TS, Holland EC (2011) Animal models for glioma drug discovery. Expert Opin Drug Discov 6: 1271-1283.

25. Hegi ME, Diserens AC, Gorlia T, Hamou MF, de Tribolet N, et al. (2005) MGMT gene silencing and benefit from temozolomide in glioblastoma. N Engl J Med 352: 997-1003.

26. Wang M (2005) The role of glucocorticoid action in the pathophysiology of the Metabolic Syndrome. Nutr Metab (Lond) 2: 3.

27. Ryken TC, McDermott M, Robinson PD, Ammirati M, Andrews DW, et al. (2010) The role of steroids in the management of brain metastases: a systematic review and evidence-based clinical practice guideline. J Neurooncol 96: 103-114. 
28. Sandi C (2004) Stress, cognitive impairment and cell adhesion molecules. Nat Rev Neurosci 5: 917-930.

29. Barnes PJ (2005) Molecular mechanisms and cellular effects of glucocorticosteroids. Immunol Allergy Clin North Am 25: 451-468.

30. Mysliwiec J, Kretowski A, Szelachowska M, Mikita A, Kinalska I (1999) Serum pro- and anti-inflammatory cytokines in patients with Graves' disease with ophthalmopathy during treatment with glucocorticoids. Rocz Akad Med Bialymst 44: $160-169$.

31. Ray A, Prefontaine KE (1994) Physical association and functional antagonism between the p65 subunit of transcription factor NF-kappa B and the glucocorticoid receptor. Proc Natl Acad Sci U S A 91: 752-756.

32. Xu J, Kim GM, Ahmed SH, Xu J, Yan P, et al. (2001) Glucocorticoid receptor-mediated suppression of activator protein-1 activation and matrix metalloproteinase expression after spinal cord injury. J Neurosci 21: 92-97.

33. Barnes PJ, Adcock IM, Ito K (2005) Histone acetylation and deacetylation: importance in inflammatory lung diseases. Eur Respir J 25: 552-563.

34. Ito K, Barnes PJ, Adcock IM (2000) Glucocorticoid receptor recruitment of histone deacetylase 2 inhibits interleukin-1 beta-induced histone $\mathrm{H} 4$ acetylation on lysines 8 and 12. Mol Cell Biol 20: 6891-6903.

35. Shapiro WR, Posner JB (1974) Corticosteroid hormones. Effects in an experimental brain tumor. Arch Neurol 30: 217-221.

36. Freshney RI, Sherry A, Hassanzadah M, Freshney M, Crilly P, et al. (1980) Control of cell proliferation in human glioma by glucocorticoids. Br J Cancer 41: 857-866.

37. Mariotta M, Perewusnyk G, Koechli OR, Little JB, von Knebel Doeberitz M, et al. (1999) Dexamethasone-induced enhancement of resistance to ionizing radiation and chemotherapeutic agents in human tumor cells. Strahlenther Onkol 175: 392-396.

38. Torlakovic E, Lilleby W, Berner A, Torlakovic G, Chibbar R, et al. (2005) Differential expression of steroid receptors in prostate tissues before and after radiation therapy for prostatic adenocarcinoma. Int J Cancer 117: 381-386.

39. Tidd DM, Paterson AR (1974) A biochemical mechanism for the delayed cytotoxic reaction of 6-mercaptopurine. Cancer Res 34: 738-746.

40. Karran P (2006) Thiopurines, DNA damage, DNA repair and therapy-related cancer. Br Med Bull 79-80: 153-170.

41. Zhou S (2006) Clinical pharmacogenomics of thiopurine S-methyltransferase. Curr Clin Pharmacol 1: 119-128. 
42. Elion GB (1989) Nobel lecture in physiology or medicine--1988. The purine path to chemotherapy. In Vitro Cell Dev Biol 25: 321-330.

43. Tai HL, Krynetski EY, Schuetz EG, Yanishevski Y, Evans WE (1997) Enhanced proteolysis of thiopurine S-methyltransferase (TPMT) encoded by mutant alleles in humans (TPMT*3A, TPMT*2): mechanisms for the genetic polymorphism of TPMT activity. Proc Natl Acad Sci U S A 94: 6444-6449.

44. Evans WE, Horner M, Chu YQ, Kalwinsky D, Roberts WM (1991) Altered mercaptopurine metabolism, toxic effects, and dosage requirement in a thiopurine methyltransferase-deficient child with acute lymphocytic leukemia. J Pediatr 119: 985-989.

45. Schutz E, Gummert J, Mohr F, Oellerich M (1993) Azathioprine-induced myelosuppression in thiopurine methyltransferase deficient heart transplant recipient. Lancet 341: 436.

46. Evans WE, Hon YY, Bomgaars L, Coutre S, Holdsworth M, et al. (2001) Preponderance of thiopurine S-methyltransferase deficiency and heterozygosity among patients intolerant to mercaptopurine or azathioprine. J Clin Oncol 19: 2293-2301.

47. Relling MV, Rubnitz JE, Rivera GK, Boyett JM, Hancock ML, et al. (1999) High incidence of secondary brain tumours after radiotherapy and antimetabolites. Lancet 354: 34-39.

48. Hijiya N, Hudson MM, Lensing S, Zacher M, Onciu M, et al. (2007) Cumulative incidence of secondary neoplasms as a first event after childhood acute lymphoblastic leukemia. Jama 297: 1207-1215.

49. Kandiel A, Fraser AG, Korelitz BI, Brensinger C, Lewis JD (2005) Increased risk of lymphoma among inflammatory bowel disease patients treated with azathioprine and 6-mercaptopurine. Gut 54: 1121-1125.

50. Hiesse C, Larue JR, Kriaa F, Blanchet P, Bellamy J, et al. (1995) Incidence and type of malignancies occurring after renal transplantation in conventionally and in cyclosporine-treated recipients: single-center analysis of a 20 -year period in 1600 patients. Transplant Proc 27: 2450-2451.

51. Guenova E, Lichte V, Hoetzenecker W, Woelbing F, Moehrle M, et al. (2009) Nodular malignant melanoma and multiple cutaneous neoplasms under immunosuppression with azathioprine. Melanoma Res 19: 271-273.

52. Snanoudj R, Durrbach A, Leblond V, Caillard S, Hurault De Ligny B, et al. (2003) Primary brain lymphomas after kidney transplantation: presentation and outcome. Transplantation 76: 930-937. 
53. Penn I (1993) Incidence and treatment of neoplasia after transplantation. The Journal of heart and lung transplantation : the official publication of the International Society for Heart Transplantation 12: S328-336.

54. Brem R, Li F, Montaner B, Reelfs O, Karran P (2010) DNA breakage and cell cycle checkpoint abrogation induced by a therapeutic thiopurine and UVA radiation.

Oncogene 29: 3953-3963.

55. Elion GB, Burgi E, Hitchings GH (1952) Studies on Condensed Pyrimidine Systems. IX. The Synthesis of Some 6-Substituted Purines. J Am Chem Soc 74: 411-414.

56. Burchenal JH, Murphy ML, Ellison RR, Sykes MP, Tan TC, et al. (1953) Clinical evaluation of a new antimetabolite, 6-mercaptopurine, in the treatment of leukemia and allied diseases. Blood 8: 965-999.

57. Walter AW, Hancock ML, Pui CH, Hudson MM, Ochs JS, et al. (1998) Secondary brain tumors in children treated for acute lymphoblastic leukemia at St Jude Children's Research Hospital. J Clin Oncol 16: 3761-3767.

58. Rosso P, Terracini B, Fears TR, Jankovic M, Fossati Bellani F, et al. (1994) Second malignant tumors after elective end of therapy for a first cancer in childhood: a multicenter study in Italy. Int J Cancer 59: 451-456.

59. Nygaard R, Garwicz S, Haldorsen T, Hertz H, Jonmundsson GK, et al. (1991) Second malignant neoplasms in patients treated for childhood leukemia. A population-based cohort study from the Nordic countries. The Nordic Society of Pediatric Oncology and Hematology (NOPHO). Acta Paediatr Scand 80: 1220-1228.

60. Salvati M, Frati A, Caroli E, Russo N, Polli FM, et al. (2003) Glioblastoma in kidney transplant recipients. Report of five cases. J Neurooncol 63: 33-37.

61. Herrlinger U, Weller M, Dichgans J, Melms A (2000) Association of primary central nervous system lymphoma with long-term azathioprine therapy for myasthenia gravis? Ann Neurol 47: 682-683.

62. Beaugerie L, Brousse N, Bouvier AM, Colombel JF, Lemann M, et al. (2009) Lymphoproliferative disorders in patients receiving thiopurines for inflammatory bowel disease: a prospective observational cohort study. Lancet 374: 1617-1625.

63. Farrell RJ, Ang Y, Kileen P, O'Briain DS, Kelleher D, et al. (2000) Increased incidence of non-Hodgkin's lymphoma in inflammatory bowel disease patients on immunosuppressive therapy but overall risk is low. Gut 47: 514-519.

64. Chen SC, Cummings OW, Hartley MP, Filomena CA, Cho WK (2006) Hepatocellular carcinoma occurring in a patient with Crohn's disease treated with both azathioprine and infliximab. Dig Dis Sci 51: 952-955. 
65. Dayharsh GA, Loftus EV, Jr., Sandborn WJ, Tremaine WJ, Zinsmeister AR, et al. (2002) Epstein-Barr virus-positive lymphoma in patients with inflammatory bowel disease treated with azathioprine or 6-mercaptopurine. Gastroenterology 122: 72-77.

66. Schmiegelow K, Al-Modhwahi I, Andersen MK, Behrendtz M, Forestier E, et al. (2009) Methotrexate/6-mercaptopurine maintenance therapy influences the risk of a second malignant neoplasm after childhood acute lymphoblastic leukemia: results from the NOPHO ALL-92 study. Blood 113: 6077-6084.

67. Vancura RW, Kepes JJ, Newell KL, Ha TM, Arnold PM (2006) Secondary intracranial neoplasms exhibiting features of astrocytoma and neuroblastoma in 2 children treated for acute lymphoblastic leukemia: report of 2 cases. Surg Neurol 65: 490-494.

68. Jones TS, Holland EC (2011) Standard of care therapy for malignant glioma and its effect on tumor and stromal cells. Oncogene.

69. Stupp R, Hegi ME, Gilbert MR, Chakravarti A (2007) Chemoradiotherapy in malignant glioma: standard of care and future directions. J Clin Oncol 25: 4127-4136.

70. Sathornsumetee S, Reardon DA, Desjardins A, Quinn JA, Vredenburgh JJ, et al. (2007) Molecularly targeted therapy for malignant glioma. Cancer 110: 13-24.

71. Furnari FB, Fenton T, Bachoo RM, Mukasa A, Stommel JM, et al. (2007) Malignant astrocytic glioma: genetics, biology, and paths to treatment. Genes Dev 21: 2683-2710.

72. Jelsma R, Bucy PC (1967) The treatment of glioblastoma multiforme of the brain. J Neurosurg 27: 388-400.

73. Badie B, Schartner JM, Paul J, Bartley BA, Vorpahl J, et al. (2000) Dexamethasone-induced abolition of the inflammatory response in an experimental glioma model: a flow cytometry study. J Neurosurg 93: 634-639.

74. Badie B, Schartner JM, Hagar AR, Prabakaran S, Peebles TR, et al. (2003) Microglia cyclooxygenase-2 activity in experimental gliomas: possible role in cerebral edema formation. Clin Cancer Res 9: 872-877.

75. Rosenberg GA (2002) Matrix metalloproteinases in neuroinflammation. Glia 39: 279-291.

76. Grasso RJ, Johnson CE, Boler RK, Moore NA (1977) Combined growth-inhibitory responses and ultrastructural alterations produced by 1,3-bis(2-chloroethyl)-1-nitrosourea and dexamethasone in rat glioma cell cultures. Cancer Res 37: 585-594. 
77. Guner M, Freshney RI, Morgan D, Freshney MG, Thomas DG, et al. (1977) Effects of dexamethasone and betamethasone on in vitro cultures from human astrocytoma. Br J Cancer 35: 439-447.

78. Li W, Graeber MB (2012) The molecular profile of microglia under the influence of glioma. Neuro Oncol 14: 958-978.

79. Fries G, Perneczky A, Kempski O (1996) Glioblastoma-associated circulating monocytes and the release of epidermal growth factor. J Neurosurg 85: 642-647.

80. Markovic DS, Glass R, Synowitz M, Rooijen N, Kettenmann H (2005) Microglia stimulate the invasiveness of glioma cells by increasing the activity of metalloprotease-2. J Neuropathol Exp Neurol 64: 754-762.

81. Dai C, Celestino JC, Okada Y, Louis DN, Fuller GN, et al. (2001) PDGF autocrine stimulation dedifferentiates cultured astrocytes and induces oligodendrogliomas and oligoastrocytomas from neural progenitors and astrocytes in vivo. Genes Dev 15: 1913-1925.

82. Hambardzumyan D, Amankulor NM, Helmy KY, Becher OJ, Holland EC (2009) Modeling Adult Gliomas Using RCAS/t-va Technology. Transl Oncol 2: 89-95.

83. Reagan-Shaw S, Nihal M, Ahmad N (2008) Dose translation from animal to human studies revisited. Faseb J 22: 659-661.

84. Nestler U, Winking M, Boker DK (2002) The tissue level of dexamethasone in human brain tumors is about 1000 times lower than the cytotoxic concentration in cell culture. Neurol Res 24: 479-482.

85. Li L, Lin X, Staver M, Shoemaker A, Semizarov D, et al. (2005) Evaluating hypoxia-inducible factor-1alpha as a cancer therapeutic target via inducible RNA interference in vivo. Cancer Res 65: 7249-7258.

86. Komohara Y, Ohnishi K, Kuratsu J, Takeya M (2008) Possible involvement of the M2 anti-inflammatory macrophage phenotype in growth of human gliomas. J Pathol 216: $15-24$.

87. Gutin PH (1977) Corticosteroid therapy in patients with brain tumors. Natl Cancer Inst Monogr 46: 151-156.

88. Wen PY, MacDonald L, Gigas DC (2005) Management of non-neoplastic problems in brain tumor patients; Wen PY, MacDonald L, Gigas DC, Black PM, Loeffler JS, editors.

89. Korshunov A, Golanov A, Sycheva R, Pronin I (1999) Prognostic value of tumour associated antigen immunoreactivity and apoptosis in cerebral glioblastomas: an analysis of 168 cases. J Clin Pathol 52: 574-580. 
90. Thomas H, Nasim MM, Sarraf CE, Alison MR, Love S, et al. (1995) Proliferating cell nuclear antigen (PCNA) immunostaining-a prognostic factor in ovarian cancer? Br J Cancer 71: 357-362.

91. Bozlu M, Orhan D, Baltaci S, Yaman O, Elhan AH, et al. (2002) The prognostic value of proliferating cell nuclear antigen, Ki-67 and nucleolar organizer region in transitional cell carcinoma of the bladder. Int Urol Nephrol 33: 59-66.

92. Weller M, Schmidt C, Roth W, Dichgans J (1997) Chemotherapy of human malignant glioma: prevention of efficacy by dexamethasone? Neurology 48: 1704-1709.

93. Gorman AM, Hirt UA, Orrenius S, Ceccatelli S (2000) Dexamethasone pre-treatment interferes with apoptotic death in glioma cells. Neuroscience 96: 417-425.

94. Rutz HP, Mariotta M, von Knebel Doeberitz M, Mirimanoff RO (1998) Dexamethasone-induced radioresistance occurring independent of human papilloma virus gene expression in cervical carcinoma cells. Strahlenther Onkol 174: 71-74.

95. Badie B, Schartner J (2001) Role of microglia in glioma biology. Microsc Res Tech 54: 106-113.

96. Tambuyzer BR, Ponsaerts P, Nouwen EJ (2009) Microglia: gatekeepers of central nervous system immunology. J Leukoc Biol 85: 352-370.

97. Rao JS (2003) Molecular mechanisms of glioma invasiveness: the role of proteases. Nat Rev Cancer 3: 489-501.

98. Coniglio SJ, Eugenin E, Dobrenis K, Stanley ER, West BL, et al. (2012) Microglial stimulation of glioblastoma invasion involves epidermal growth factor receptor (EGFR) and colony stimulating factor 1 receptor (CSF-1R) signaling. Mol Med 18: 519-527.

99. Wu G, Morris SM, Jr. (1998) Arginine metabolism: nitric oxide and beyond. Biochem J 336 ( Pt 1): 1-17.

100. Arend WP, Malyak M, Guthridge CJ, Gabay C (1998) Interleukin-1 receptor antagonist: role in biology. Annu Rev Immunol 16: 27-55.

101. Chandrasekar N, Jasti S, Alfred-Yung WK, Ali-Osman F, Dinh DH, et al. (2000) Modulation of endothelial cell morphogenesis in vitro by MMP-9 during glial-endothelial cell interactions. Clin Exp Metastasis 18: 337-342.

102. Itoh T, Tanioka M, Matsuda H, Nishimoto H, Yoshioka T, et al. (1999) Experimental metastasis is suppressed in MMP-9-deficient mice. Clin Exp Metastasis 17: 177-181. 
103. Coussens LM, Tinkle CL, Hanahan D, Werb Z (2000) MMP-9 supplied by bone marrow-derived cells contributes to skin carcinogenesis. Cell 103: 481-490.

104. Bergers G, Brekken R, McMahon G, Vu TH, Itoh T, et al. (2000) Matrix metalloproteinase-9 triggers the angiogenic switch during carcinogenesis. Nat Cell Biol 2: 737-744.

105. Weinshilboum RM, Raymond FA, Pazmino PA (1978) Human erythrocyte thiopurine methyltransferase: radiochemical microassay and biochemical properties. Clin Chim Acta 85: 323-333.

106. Weinshilboum RM, Sladek SL (1980) Mercaptopurine pharmacogenetics: monogenic inheritance of erythrocyte thiopurine methyltransferase activity. Am J Hum Genet 32: 651-662.

107. Krynetski EY, Schuetz JD, Galpin AJ, Pui CH, Relling MV, et al. (1995) A single point mutation leading to loss of catalytic activity in human thiopurine S-methyltransferase. Proc Natl Acad Sci U S A 92: 949-953.

108. Krynetski E, Evans WE (2003) Drug methylation in cancer therapy: lessons from the TPMT polymorphism. Oncogene 22: 7403-7413.

109. Krynetski EY, Fessing MY, Yates CR, Sun D, Schuetz JD, et al. (1997) Promoter and intronic sequences of the human thiopurine S-methyltransferase (TPMT) gene isolated from a human PAC1 genomic library. Pharm Res 14: 1672-1678.

110. Szumlanski C, Otterness D, Her C, Lee D, Brandriff B, et al. (1996) Thiopurine methyltransferase pharmacogenetics: human gene cloning and characterization of a common polymorphism. DNA Cell Biol 15: 17-30.

111. Honchel R, Aksoy IA, Szumlanski C, Wood TC, Otterness DM, et al. (1993) Human thiopurine methyltransferase: molecular cloning and expression of T84 colon carcinoma cell cDNA. Mol Pharmacol 43: 878-887.

112. Warren DJ, Andersen A, Slordal L (1995) Quantitation of 6-thioguanine residues in peripheral blood leukocyte DNA obtained from patients receiving 6-mercaptopurine-based maintenance therapy. Cancer Res 55: 1670-1674.

113. Cuffari C, Li DY, Mahoney J, Barnes Y, Bayless TM (2004) Peripheral blood mononuclear cell DNA 6-thioguanine metabolite levels correlate with decreased interferon-gamma production in patients with Crohn's disease on AZA therapy. Dig Dis Sci 49: 133-137.

114. Ling YH, Chan JY, Beattie KL, Nelson JA (1992) Consequences of 6-thioguanine incorporation into DNA on polymerase, ligase, and endonuclease reactions. Mol Pharmacol 42: 802-807. 
115. Bodell WJ (1991) Molecular dosimetry of sister chromatid exchange induction in 9L cells treated with 6-thioguanine. Mutagenesis 6: 175-177.

116. Christie NT, Drake S, Meyn RE, Nelson JA (1984) 6-Thioguanine-induced DNA damage as a determinant of cytotoxicity in cultured Chinese hamster ovary cells. Cancer Res 44: 3665-3671.

117. Pan BF, Nelson JA (1990) Characterization of the DNA damage in 6-thioguanine-treated cells. Biochem Pharmacol 40: 1063-1069.

118. Lennard L, Van Loon JA, Lilleyman JS, Weinshilboum RM (1987) Thiopurine pharmacogenetics in leukemia: correlation of erythrocyte thiopurine methyltransferase activity and 6-thioguanine nucleotide concentrations. Clin Pharmacol Ther 41: 18-25.

119. Ford LT, Berg JD (2010) Thiopurine S-methyltransferase (TPMT) assessment prior to starting thiopurine drug treatment; a pharmacogenomic test whose time has come. J Clin Pathol 63: 288-295.

120. Yates CR, Krynetski EY, Loennechen T, Fessing MY, Tai HL, et al. (1997) Molecular diagnosis of thiopurine S-methyltransferase deficiency: genetic basis for azathioprine and mercaptopurine intolerance. Ann Intern Med 126: 608-614.

121. Otterness D, Szumlanski C, Lennard L, Klemetsdal B, Aarbakke J, et al. (1997) Human thiopurine methyltransferase pharmacogenetics: gene sequence polymorphisms. Clin Pharmacol Ther 62: 60-73.

122. Tai HL, Krynetski EY, Yates CR, Loennechen T, Fessing MY, et al. (1996) Thiopurine S-methyltransferase deficiency: two nucleotide transitions define the most prevalent mutant allele associated with loss of catalytic activity in Caucasians. Am J Hum Genet 58: 694-702.

123. Spire-Vayron de la Moureyre C, Debuysere H, Sabbagh N, Marez D, Vinner E, et al. (1998) Detection of known and new mutations in the thiopurine S-methyltransferase gene by single-strand conformation polymorphism analysis. Hum Mutat 12: 177-185.

124. Otterness DM, Szumlanski CL, Wood TC, Weinshilboum RM (1998) Human thiopurine methyltransferase pharmacogenetics. Kindred with a terminal exon splice junction mutation that results in loss of activity. J Clin Invest 101: 1036-1044.

125. Tai HL, Fessing MY, Bonten EJ, Yanishevsky Y, d'Azzo A, et al. (1999) Enhanced proteasomal degradation of mutant human thiopurine S-methyltransferase (TPMT) in mammalian cells: mechanism for TPMT protein deficiency inherited by TPMT*2, TPMT*3A, TPMT*3B or TPMT*3C. Pharmacogenetics 9: 641-650. 
126. Hon YY, Fessing MY, Pui CH, Relling MV, Krynetski EY, et al. (1999) Polymorphism of the thiopurine S-methyltransferase gene in African-Americans. Hum Mol Genet 8: 371-376.

127. Schaeffeler E, Fischer C, Brockmeier D, Wernet D, Moerike K, et al. (2004) Comprehensive analysis of thiopurine S-methyltransferase phenotype-genotype correlation in a large population of German-Caucasians and identification of novel TPMT variants. Pharmacogenetics 14: 407-417.

128. Rossi AM, Bianchi M, Guarnieri C, Barale R, Pacifici GM (2001) Genotype-phenotype correlation for thiopurine S-methyltransferase in healthy Italian subjects. Eur J Clin Pharmacol 57: 51-54.

129. Hakooz N, Arafat T, Payne D, Ollier W, Pushpakom S, et al. (2010) Genetic analysis of thiopurine methyltransferase polymorphism in the Jordanian population. Eur J Clin Pharmacol 66: 999-1003.

130. Collie-Duguid ES, Pritchard SC, Powrie RH, Sludden J, Collier DA, et al. (1999) The frequency and distribution of thiopurine methyltransferase alleles in Caucasian and Asian populations. Pharmacogenetics 9: 37-42.

131. Hamdy SI, Hiratsuka M, Narahara K, Endo N, El-Enany M, et al. (2003) Genotype and allele frequencies of TPMT, NAT2, GST, SULT1A1 and MDR-1 in the Egyptian population. Br J Clin Pharmacol 55: 560-569.

132. Ameyaw MM, Collie-Duguid ES, Powrie RH, Ofori-Adjei D, McLeod HL (1999) Thiopurine methyltransferase alleles in British and Ghanaian populations. Hum Mol Genet 8: 367-370.

133. Mircheva J, Legendre C, Soria-Royer C, Thervet E, Beaune P, et al. (1995) Monitoring of azathioprine-induced immunosuppression with thiopurine methyltransferase activity in kidney transplant recipients. Transplantation 60 : 639-642.

134. Szumlanski CL, Weinshilboum RM (1995) Sulphasalazine inhibition of thiopurine methyltransferase: possible mechanism for interaction with 6-mercaptopurine and azathioprine. Br J Clin Pharmacol 39: 456-459.

135. Anstey A, Lennard L, Mayou SC, Kirby JD (1992) Pancytopenia related to azathioprine--an enzyme deficiency caused by a common genetic polymorphism: a review. J R Soc Med 85: 752-756.

136. Lennard L, Maddocks JL (1983) Assay of 6-thioguanine nucleotide, a major metabolite of azathioprine, 6-mercaptopurine and 6-thioguanine, in human red blood cells. J Pharm Pharmacol 35: 15-18. 
137. Lennard L, Lilleyman JS, Van Loon J, Weinshilboum RM (1990) Genetic variation in response to 6-mercaptopurine for childhood acute lymphoblastic leukaemia. Lancet 336: 225-229.

138. Balis FM, Holcenberg JS, Poplack DG, Ge J, Sather HN, et al. (1998) Pharmacokinetics and pharmacodynamics of oral methotrexate and mercaptopurine in children with lower risk acute lymphoblastic leukemia: a joint children's cancer group and pediatric oncology branch study. Blood 92: 3569-3577.

139. Relling MV, Hancock ML, Boyett JM, Pui CH, Evans WE (1999) Prognostic importance of 6-mercaptopurine dose intensity in acute lymphoblastic leukemia. Blood 93: 2817-2823.

140. Schmiegelow K, Bruunshuus I (1990) 6-Thioguanine nucleotide accumulation in red blood cells during maintenance chemotherapy for childhood acute lymphoblastic leukemia, and its relation to leukopenia. Cancer Chemother Pharmacol 26: 288-292.

141. Lennard L, Lilleyman JS (1996) Individualizing therapy with 6-mercaptopurine and 6-thioguanine related to the thiopurine methyltransferase genetic polymorphism. Ther Drug Monit 18: 328-334.

142. Evans WE, Relling MV (2004) Moving towards individualized medicine with pharmacogenomics. Nature 429: 464-468.

143. Lilleyman JS, Lennard L (1994) Mercaptopurine metabolism and risk of relapse in childhood lymphoblastic leukaemia. Lancet 343: 1188-1190.

144. Dong XW, Zheng Q, Zhu MM, Tong JL, Ran ZH (2010) Thiopurine S-methyltransferase polymorphisms and thiopurine toxicity in treatment of inflammatory bowel disease. World J Gastroenterol 16: 3187-3195.

145. Kurzawski M, Dziewanowski K, Lener A, Drozdzik M (2009) TPMT but not ITPA gene polymorphism influences the risk of azathioprine intolerance in renal transplant recipients. Eur J Clin Pharmacol 65: 533-540.

146. Overall RW, Kempermann G, Peirce J, Lu L, Goldowitz D, et al. (2009) Genetics of the hippocampal transcriptome in mouse: a systematic survey and online neurogenomics resource. Front Neurosci 3: 55.

147. Szumlanski CL, Honchel R, Scott MC, Weinshilboum RM (1992) Human liver thiopurine methyltransferase pharmacogenetics: biochemical properties, liver-erythrocyte correlation and presence of isozymes. Pharmacogenetics 2: 148-159.

148. Van Loon JA, Weinshilboum RM (1982) Thiopurine methyltransferase biochemical genetics: human lymphocyte activity. Biochemical genetics 20: 637-658. 
149. Van Loon JA, Szumlanski CL, Weinshilboum RM (1992) Human kidney thiopurine methyltransferase. Photoaffinity labeling with S-adenosyl-L-methionine. Biochem Pharmacol 44: 775-785.

150. McLeod HL, Relling MV, Liu Q, Pui CH, Evans WE (1995) Polymorphic thiopurine methyltransferase in erythrocytes is indicative of activity in leukemic blasts from children with acute lymphoblastic leukemia. Blood 85: 1897-1902.

151. Yuan B, O'Connor TR, Wang Y (2010) 6-Thioguanine and S-methylthioguanine are mutagenic in human cells. ACS chemical biology 5: 1021-1027.

152. Karran P, Attard N (2008) Thiopurines in current medical practice: molecular mechanisms and contributions to therapy-related cancer. Nat Rev Cancer 8: 24-36.

153. Yuan B, Wang Y (2008) Mutagenic and cytotoxic properties of 6-thioguanine, S6-methylthioguanine, and guanine-S6-sulfonic acid. J Biol Chem 283: 23665-23670.

154. Yue J, Sheng Y, Ren A, Penmatsa S (2010) A miR-21 hairpin structure-based gene knockdown vector. Biochem Biophys Res Commun 394: 667-672.

155. Katyal S, el-Khamisy SF, Russell HR, Li Y, Ju L, et al. (2007) TDP1 facilitates chromosomal single-strand break repair in neurons and is neuroprotective in vivo. EMBO J 26: 4720-4731.

156. Yan T, Berry SE, Desai AB, Kinsella TJ (2003) DNA mismatch repair (MMR) mediates 6-thioguanine genotoxicity by introducing single-strand breaks to signal a G2-M arrest in MMR-proficient RKO cells. Clin Cancer Res 9: 2327-2334.

157. Hosni-Ahmed A, Barnes JD, Wan J, Jones TS (2011) Thiopurine methyltransferase predicts the extent of cytotoxicty and DNA damage in astroglial cells after thioguanine exposure. PLoS ONE 6: e29163.

158. Hartford C, Vasquez E, Schwab M, Edick MJ, Rehg JE, et al. (2007) Differential effects of targeted disruption of thiopurine methyltransferase on mercaptopurine and thioguanine pharmacodynamics. Cancer Res 67: 4965-4972.

159. Olive PL, Banath JP (2006) The comet assay: a method to measure DNA damage in individual cells. Nat Protoc 1: 23-29.

160. Kinsella TJ (2009) Coordination of DNA mismatch repair and base excision repair processing of chemotherapy and radiation damage for targeting resistant cancers. Clin Cancer Res 15: 1853-1859.

161. Chalastanis A, Penard-Lacronique V, Svrcek M, Defaweux V, Antoine N, et al. (2010) Azathioprine-induced carcinogenesis in mice according to Msh2 genotype. J Natl Cancer Inst 102: 1731-1740. 
162. Cooley N, Elder RH, Povey AC (2010) The effect of Msh2 knockdown on methylating agent induced toxicity in DNA glycosylase deficient cells. Toxicology 268: 111-117.

163. Karran P (2001) Mechanisms of tolerance to DNA damaging therapeutic drugs. Carcinogenesis 22: 1931-1937.

164. Stanulla M, Schaeffeler E, Moricke A, Coulthard SA, Cario G, et al. (2009) Thiopurine methyltransferase genetics is not a major risk factor for secondary malignant neoplasms after treatment of childhood acute lymphoblastic leukemia on Berlin-Frankfurt-Munster protocols. Blood 114: 1314-1318.

165. Mohler ML, Kang GS, Hong SS, Patil R, Kirichenko OV, et al. (2006) Discovery of antiglioma activity of biaryl 1,2,3,4-tetrahydroisoquinoline derivatives and conformationally flexible analogues. J Med Chem 49: 5845-5848.

166. Patil R, Hosni-Ahmed A, Jones T, Patil S, Asres L, et al. (2014) Synthesis and In Vitro Evaluation of Novel 1,2,3,4-Tetrahydroisoquinoline Derivatives as Potent Antiglioma Agents. Anticancer Agents Med Chem (In Press).

167. Sugahara KN, Teesalu T, Karmali PP, Kotamraju VR, Agemy L, et al. (2010) Coadministration of a tumor-penetrating peptide enhances the efficacy of cancer drugs. Science 328: 1031-1035.

168. Patil SA, Wang J, Li XS, Chen J, Jones TS, et al. (2012) New substituted 4H-chromenes as anticancer agents. Bioorg Med Chem Lett 22: 4458-4461.

169. Giri A, Lakshmi Narasu M (2000) Production of podophyllotoxin from Podophyllum hexandrum: a potential natural product for clinically useful anticancer drugs. Cytotechnology 34: 17-26.

170. Cai SX, Drewe J, Kemnitzer W (2009) Discovery of 4-aryl-4H-chromenes as potent apoptosis inducers using a cell- and caspase-based Anti-cancer Screening Apoptosis Program (ASAP): SAR studies and the identification of novel vascular disrupting agents. Anticancer Agents Med Chem 9: 437-456.

171. Kemnitzer W, Drewe J, Jiang S, Zhang H, Crogan-Grundy C, et al. (2008) Discovery of 4-aryl-4H-chromenes as a new series of apoptosis inducers using a cell- and caspase-based high throughput screening assay. 4. Structure-activity relationships of $\mathrm{N}$-alkyl substituted pyrrole fused at the 7,8-positions. J Med Chem 51: 417-423.

172. Khalil AA, Jameson MJ, Broaddus WC, Lin PS, Dever SM, et al. (2013) The Influence of Hypoxia and $\mathrm{pH}$ on Bioluminescence Imaging of Luciferase-Transfected Tumor Cells and Xenografts. Int J Mol Imaging 2013: 287697. 
173. Zhu XK, Guan J, Tachibana Y, Bastow KF, Cho SJ, et al. (1999) Antitumor agents. 194. Synthesis and biological evaluations of 4-beta-mono-, -di-, and -trisubstituted aniline-4'-O-demethyl-podophyllotoxin and related compounds with improved pharmacological profiles. J Med Chem 42: 2441-2446.

174. Subrahmanyam D, Renuka B, Kumar GS, Vandana V, Deevi DS (1999) 9-Deoxopodophyllotoxin derivatives as anti-cancer agents. Bioorg Med Chem Lett 9: 2131-2134.

175. Damayanthi Y, Lown JW (1998) Podophyllotoxins: current status and recent developments. Curr Med Chem 5: 205-252.

176. Chen JY, Tang YA, Li WS, Chiou YC, Shieh JM, et al. (2013) A synthetic podophyllotoxin derivative exerts anti-cancer effects by inducing mitotic arrest and pro-apoptotic ER stress in lung cancer preclinical models. PLoS One 8: e62082.

177. Kamal A, Suresh P, Ramaiah MJ, Srinivasa Reddy T, Kapavarapu RK, et al. (2013) 4beta-[4'-(1-(Aryl)ureido)benzamide]podophyllotoxins as DNA topoisomerase I and IIalpha inhibitors and apoptosis inducing agents. Bioorg Med Chem 21: 5198-5208.

178. Song P, Ma F, Wang F, Wang X, Patil R, et al. (2008) Plasma and cerebrospinal fluid pharmacokinetics of the novel tetrahydroisoquinoline EDL-155 in rats. Cancer Chemother Pharmacol 61: 1037-1044.

179. Sui M, Chen F, Chen Z, Fan W (2006) Glucocorticoids interfere with therapeutic efficacy of paclitaxel against human breast and ovarian xenograft tumors. Int J Cancer 119: 712-717.

180. Chao CC, Hu S, Close K, Choi CS, Molitor TW, et al. (1992) Cytokine release from microglia: differential inhibition by pentoxifylline and dexamethasone. J Infect Dis 166: 847-853.

181. Jun CD, Hoon R, Um JY, Kim TY, Kim JM, et al. (1994) Involvement of protein kinase $\mathrm{C}$ in the inhibition of nitric oxide production from murine microglial cells by glucocorticoid. Biochem Biophys Res Commun 199: 633-638.

182. Minghetti L, Nicolini A, Polazzi E, Greco A, Perretti M, et al. (1999) Down-regulation of microglial cyclo-oxygenase-2 and inducible nitric oxide synthase expression by lipocortin 1. Br J Pharmacol 126: 1307-1314.

183. da Fonseca AC, Badie B (2013) Microglia and macrophages in malignant gliomas: recent discoveries and implications for promising therapies. Clin Dev Immunol 2013: 264124. 


\section{VITA}

Amira Ahmed was born 1982 in Fayoum, Egypt. She completed her Bachelor's degree (honors) in Chemistry and Biochemistry in 2003 from College of Sciences, University of Fayoum, Egypt. In 2007, She earned her Master's degree (honors) in Biochemistry from the University of Fayoum. In June 2007, she joined VAMC, Memphis as a visiting scholar after she was awarded the Partnership and Ownership Initiative Scholarship from Egyptian Ministry of Higher Education and State for Scientific Research. In November 2008, she Joined Dr. Terreia Jones lab as a senior research assistant until she joined the Pharmaceutical Sciences Graduate Program in August 2009 under the supervision of Dr. Jones. In February 2012 and upon Jones leaving UTHSC, Drs. Duane Miller and Lawrence Pfeffer have served as co-mentors. During her PhD she was studying glioma in relation to drug induced toxicity and resistance. 